\title{
New Electrophilic Difluoromethylating Reagent
}

\author{
G. K. Surya Prakash, ${ }^{*}$ Csaba Weber, Sujith Chacko \\ and George A. Olah* \\ Loker Hydrocarbon Research Institute and Department of Chemistry, \\ University of Southern California, Los Angeles, California 90089- \\ 1661 \\ gprakash@usc.edu; olah@usc.edu
}

\section{Supporting Information}

\section{Table of Contents}

General

Experimental section

Copy of spectra
S1-S2

S2-S16

S17-S112

\section{General:}

Unless otherwise mentioned, all reagents were purchased from commercial sources. Dichloromethane was distilled under nitrogen over calcium hydride or was used as received from Aldrich (water content $<50 \mathrm{ppm}$ ). Acetonitrile was distilled under nitrogen over phosphorus pentoxide or dry acetonitrile (water content $<50 \mathrm{ppm}$ ) was used as received from Aldrich. Diethyl ether was distilled under nitrogen over sodium.

${ }^{1} \mathrm{H},{ }^{13} \mathrm{C},{ }^{19} \mathrm{~F}$ and ${ }^{31} \mathrm{P}$ NMR spectra were recorded on Varian Mercury-400 MHz NMR spectrometer. ${ }^{1} \mathrm{H}-\mathrm{NMR}$ chemical shifts were determined relative to internal $\left(\mathrm{CH}_{3}\right)_{4} \mathrm{Si}$ 
(TMS) at $0.00 \mathrm{ppm} .{ }^{13} \mathrm{C}-\mathrm{NMR}$ chemical shifts were determined relative to internal TMS at $0.00 \mathrm{ppm} . \mathrm{CFCl}_{3}$ was used as internal standard for ${ }^{19} \mathrm{~F}$ and $\mathrm{H}_{3} \mathrm{PO}_{4}$ for ${ }^{31} \mathrm{P} \mathrm{NMR}$. High resolution mass spectrum data were recorded in the EI mode on a high resolution mass spectrometer.

Abbreviations: $\quad$ TDA: $\quad \operatorname{tris}[2-(2-m e t h o x y e t h o x y)$ ethyl]amine, $\quad m$-CPBA: 3Chloroperbenzoic acid, DCM: dichloromethane, DMSO: dimethylsulfoxide, DIAD: diisopropyl azodicarboxylate, dec.: decomposition.

\section{Experimental section}

\section{Preparation of 2-(Difluoromethylsulfanyl)bromobenzene (4). 2-} Sulfanylbromobenzene (3) (10 g, $0.053 \mathrm{~mol})$ was dissolved in hexane (50 mL). Finely powdered $\mathrm{NaOH}$ (5.3 g, 0.1325 mol, powdered in glove box) and TDA (0.85 g, 2.6 mmol) were added at room temperature under argon. The reaction mixture was warmed up to $70{ }^{\circ} \mathrm{C}$, a balloon was attached to the reaction vessel and $\mathrm{CF}_{2} \mathrm{HCl}$ gas was bubbled into the stirred mixture from a cylinder for $16 \mathrm{~h}$. The reaction was monitored by GC-MS. When all of the starting material was converted into the product, the mixture was cooled down to room temperature. Sodium carbonate solution in water $(10 \%, 50 \mathrm{~mL})$ was added, the organic layer was separated and washed again with sodium carbonate solution then it was washed with water $(50 \mathrm{~mL})$. The hexane phase was dried over anhydrous $\mathrm{MgSO}_{4}$, the drying agent was filtered off and the solvent was removed in vacuum. The crude product was distilled in vacuum $\left(20 \mathrm{Hgmm}, 64{ }^{\circ} \mathrm{C}\right)$ to give $9.8 \mathrm{~g}$ of the product as a 
colorless oil. Yield: 78\%. ${ }^{1} \mathrm{H}-\mathrm{NMR}\left(\mathrm{CDCl}_{3}\right)$ : $6.87\left(\mathrm{t}, 1 \mathrm{H}, J_{\mathrm{H}-\mathrm{F}}=57.50 \mathrm{~Hz}\right), 7.20-7.26(\mathrm{~m}$, 1H), 7.28-7.34 (m, 1H), 7.60-7.68 (m, 2H). ${ }^{19}$ F-NMR $\left(\mathrm{CDCl}_{3}\right)$ : -92.65 (d, $J_{\mathrm{H}-\mathrm{F}}=57.50$ Hz). ${ }^{13} \mathrm{C}-\mathrm{NMR}\left(\mathrm{CDCl}_{3}\right): 120.6\left(\mathrm{t}, J_{\mathrm{C}-\mathrm{F}}=277 \mathrm{~Hz}\right), 128.50,128.52,129.3,131.2,134.0$, 136.6. GC-MS (EI, $m / z): 239\left(\mathrm{M}^{+}\right)$.

Preparation of 2-(Difluoromethylsulfanyl)biphenyl (5). A mixture of 2(difluoromethylsulfanyl)bromobenzene (4) (1.07 g, $4.47 \mathrm{mmol}$ ), phenylboronic acid (600 mg, $4.92 \mathrm{mmol})$, sodium carbonate $(531 \mathrm{mg}, \quad 4.9 \mathrm{mmol})$ and tetrakis(triphenylphosphine)palladium (0) $(210 \mathrm{mg}, 0.19 \mathrm{mmol})$ in ethanol $(5 \mathrm{~mL})$ was stirred at $100^{\circ} \mathrm{C}$ for 1 hour in a pressure tube in a microwave equipment (CEM). The solid was filtered off, the solvent was removed in vacuum and the residue was purified by silica gel column chromatography (eluent: $0.1 \%$ ethyl acetate in hexane) to give $675 \mathrm{mg}$ of the expected compound as a colorless oil. Yield: 64\%. ${ }^{1} \mathrm{H}-\mathrm{NMR}\left(\mathrm{CDCl}_{3}\right)$ : 6.66 (t, $J_{\mathrm{H}-\mathrm{F}}$ $=56.8 \mathrm{~Hz}, 1 \mathrm{H}), 7.3-7.5(\mathrm{~m}, 8 \mathrm{H}), 7.72(\mathrm{~d}, 1 \mathrm{H}, J=7.9 \mathrm{~Hz}) .{ }^{19} \mathrm{~F}-\mathrm{NMR}\left(\mathrm{CDCl}_{3}\right):-91.95(\mathrm{~d}$, $\left.J_{\mathrm{H}-\mathrm{F}}=56.8 \mathrm{~Hz}\right) .{ }^{13} \mathrm{C}-\mathrm{NMR}\left(\mathrm{CDCl}_{3}\right): 121.1\left(\mathrm{t}, J_{\mathrm{C}-\mathrm{F}}=275 \mathrm{~Hz}\right), 125.5,127.8,128.1,128.3$, 129.6, 129.7, 131.2, 135.6, 140.5, 146.6. GC-MS (EI, m/z): $236\left(\mathrm{M}^{+}\right)$. HRMS calculated for $\mathrm{C}_{13} \mathrm{H}_{10} \mathrm{~F}_{2} \mathrm{~S}$. Expected: 236.0471. Found: 236.0477.

\section{Preparation of 2-(Difluoromethylsulfinyl)bromobenzene (6). 2-}

(Difluoromethylsulfanyl)bromobenzene (4, $1.05 \mathrm{~g}, 4.4 \mathrm{mmol}$ ) was dissolved in DCM (8 mL). $m$-CPBA (1.52 g, 70\%, $6 \mathrm{mmol}$ ) was added at $0-3{ }^{\circ} \mathrm{C}$ in four portions each dissolved in DCM (4 mL) over a period of 24h. The reaction mixture was diluted with cold DCM (100 mL) and extracted with sodium carbonate solution $(20 \%, 2 \times 50 \mathrm{~mL})$. 
The organic layer was separated and dried over anhydrous magnesium sulfate. The solvent was evaporated in vacuum to give $0.95 \mathrm{~g}$ of the crude product as a white solid (GC-MS purity: 92\%). The crude product was recrystallized from $n$-hexane to yield 806 mg product having 97\% GC purity. Yield: $72 \% . \mathrm{mp}=62-63{ }^{\circ} \mathrm{C} .{ }^{1} \mathrm{H}-\mathrm{NMR}\left(\mathrm{CDCl}_{3}\right): 6.29$ (t, $\left.1 \mathrm{H}, J_{\mathrm{H}-\mathrm{F}}=54.5 \mathrm{~Hz}\right), 7.40-7.50(\mathrm{~m}, 1 \mathrm{H}), 7.55-7.64(\mathrm{~m}, 2 \mathrm{H}), 7.84-7.90(\mathrm{~m}, 1 \mathrm{H}) .{ }^{19} \mathrm{~F}-$ NMR (CDCl $)$ ) - $-115.95\left(\mathrm{dd}, J_{\mathrm{H}-\mathrm{F}}=54.5 \mathrm{~Hz}, J_{\mathrm{F}-\mathrm{F}}=250.2 \mathrm{~Hz}\right),-123.00\left(\mathrm{dd}, J_{\mathrm{H}-\mathrm{F}}=54.5 \mathrm{~Hz}\right.$,

$\left.J_{\mathrm{F}-\mathrm{F}}=250.2 \mathrm{~Hz}\right) \cdot{ }^{13} \mathrm{C}-\mathrm{NMR}\left(\mathrm{CDCl}_{3}\right): 120.19,120.25\left(\mathrm{t}, J_{\mathrm{C}-\mathrm{F}}=294 \mathrm{~Hz}\right), 120.3,128.0$, 128.9, 133.5, 134.0. HRMS calculated for $\mathrm{C}_{7} \mathrm{H}_{5} \mathrm{BrF}_{2} \mathrm{~S}$. Expected: 253.9213. Found: 253.9203.

\section{Preparation of 2-(Difluoromethylsulfinyl)biphenyl (7). Method A: 2-} (Difluoromethylsulfanyl)biphenyl (5, $420 \mathrm{mg}, 1.78 \mathrm{mmol}$ ) was dissolved in DCM (7 $\mathrm{mL}$ ). $\mathrm{m}$-CPBA (440 mg, 70\%, $1.80 \mathrm{mmol}$ ) was added at $0-3{ }^{\circ} \mathrm{C}$ in four portions - each dissolved in DCM (3mL) - over a period of $12 \mathrm{~h}$. The reaction mixture was diluted with cold DCM (50 mL) and extracted with sodium carbonate solution $(20 \%, 2 \times 20 \mathrm{~mL})$. The organic layer was separated and dried over anhydrous magnesium sulfate. The solvent was evaporated in vacuum to give $413 \mathrm{mg}$ of the expected product as a white solid. Yield: $92 \% . \mathrm{mp}=69-70{ }^{\circ} \mathrm{C}$.

Method B: A mixture of 2-(difluoromethylsulfinyl)bromobenzene (500 mg, $1.96 \mathrm{mmol}$, 4), phenylboronic acid (250 mg, $2.05 \mathrm{mmol}$ ), sodium carbonate (210 mg, $1.96 \mathrm{mmol}$ ) and tetrakis(triphenylphosphine)palladium $(0)(100 \mathrm{mg}, 0.09 \mathrm{mmol})$ in ethanol $(5 \mathrm{~mL})$ was stirred at $90{ }^{\circ} \mathrm{C}$ for $40 \mathrm{~min}$ in a pressure tube in a microwave equipment (CEM). The solid was filtered off, the solvent was removed in vacuum and the residue was purified by 
silica gel column chromatography (eluent: $0.1 \%$ ethyl acetate in hexane) to give $288 \mathrm{mg}$ of the expected compound as a white solid. Yield: $56 \%$. mp $=68-69{ }^{\circ} \mathrm{C}$.

${ }^{1} \mathrm{H}-\mathrm{NMR}\left(\mathrm{CDCl}_{3}\right)$ : $5.83\left(\mathrm{t}, 1 \mathrm{H}, J_{\mathrm{H}-\mathrm{F}}=54.90 \mathrm{~Hz}\right), 7.20-7.30(\mathrm{~m}, 2 \mathrm{H}), 7.30-7.44(\mathrm{~m}, 4 \mathrm{H})$, 7.50-7.64 (m, 2H) 7.96-8.06 (m, 1H). ${ }^{19}$ F-NMR $\left(\mathrm{CDCl}_{3}\right)$ : -116.27 (dd, $J_{\mathrm{H}-\mathrm{F}}=54.9 \mathrm{~Hz}, J_{\mathrm{F}-\mathrm{F}}$ $=254.8 \mathrm{~Hz}),-121.66\left(\mathrm{dd}, J_{\mathrm{H}-\mathrm{F}}=54.9 \mathrm{~Hz}, J_{\mathrm{F}-\mathrm{F}}=254.8 \mathrm{~Hz}\right) \cdot{ }^{13} \mathrm{C}-\mathrm{NMR}\left(\mathrm{CDCl}_{3}\right): 121.1(\mathrm{t}$, $\left.J_{\mathrm{C}-\mathrm{F}}=290.7 \mathrm{~Hz}\right), 125.7,128.7,128.9,129.0,129.6,131.1,132.6,135.5,137.2,142.4$. GC-MS (EI, m/z): $252\left(\mathrm{M}^{+}\right)$. HRMS calculated for $\mathrm{C}_{13} \mathrm{H}_{10} \mathrm{~F}_{2} \mathrm{OS}$. Expected: 252.0420 . Found: 252.0430 .

\section{Reaction of 2-(Difluoromethylsulfinyl)biphenyl (7) with Trifluoromethanesulfonic}

Anhydride. A: Experiment was carried out at 0- $20{ }^{\circ} \mathrm{C} .7$ (150 mg, $0.6 \mathrm{mmol}$ ) was dissolved in DCM (2mL), the solution was cooled to $0{ }^{\circ} \mathrm{C}$ under argon and trifluoromethanesulfonic anhydride (170 mg, $0.6 \mathrm{mmol}$ ) was added drop wise. The reaction mixture was allowed to warm up to room temperature. The reaction mixture was analyzed by ${ }^{19} \mathrm{~F}$ NMR. Difluoromethyl trifluoromethanesulfonate $(\mathbf{1 0})^{1}$ was identified as the major fluorine containing product. ${ }^{19} \mathrm{~F}$ NMR $\left(\mathrm{CH}_{2} \mathrm{Cl}_{2}\right):-74.84$ (9) (t, $\left.J_{\mathrm{F}-\mathrm{F}}=3.05 \mathrm{~Hz}\right)$, $82.43\left(\mathrm{dq}, J_{\mathrm{F}-\mathrm{H}}=68 \mathrm{~Hz}, J_{\mathrm{F}-\mathrm{F}}=3.05 \mathrm{~Hz}\right)$ The $\mathrm{DCM}$ was removed in vacuum. The residue was identified as a mixture of dibenzothiophene (9) and trifluoromethanesulfonic acid. The mixture was dissolved in DCM, extracted with water and dried over anhydrous $\mathrm{MgSO}_{4}$.The drying agent was filtered off and the solvent was removed in vacuum to give 9 as a white solid. Yield: 87\%. mp.: 87-90 ${ }^{\circ} \mathrm{C}{ }^{1} \mathrm{H}-\mathrm{NMR}\left(\mathrm{CDCl}_{3}\right)$ : 7.2-7.4 (m, 4H), 7.7-7.9 (m, 2H), 8.0-8.2 (m, 2H). ${ }^{13} \mathrm{C}-\mathrm{NMR}\left(\mathrm{CDCl}_{3}\right)$ : 121.7, 122.9, 124.4, 126.8, 135.6, 139.5. B:

\footnotetext{
${ }^{1}$ Mitsch, R. A.; Robertson, J. E. J. Heterocyclic Chemistry, 1965, 152-156.
} 
Experiment carried out at $-78{ }^{\circ} \mathrm{C}: 7$ (50 mg, $\left.0.2 \mathrm{mmol}\right)$ was dissolved in DCM $(1 \mathrm{~mL})$ in an NMR tube. The solution was cooled to $-78{ }^{\circ} \mathrm{C}$ under argon and trifluoromethanesulfonic anhydride (54 mg, $0.2 \mathrm{mmol}$ ) was added drop wise. The reaction mixture was analyzed by low temperature ${ }^{19} \mathrm{~F}-\mathrm{NMR}$ at $-78{ }^{\circ} \mathrm{C}$ : -71.4 (s) [trifluoromethanesulfonic anhydride, starting material], -78.8 (s) [triflic acid], -101.6 (d, $\left.J_{\mathrm{H}-\mathrm{F}}=56.8 \mathrm{~Hz}\right)\left[8\right.$, product], $-116.8\left(\mathrm{~d}, J_{\mathrm{F}-\mathrm{F}}=268 \mathrm{~Hz}\right),-121.7\left(\mathrm{~d}, J_{\mathrm{F}-\mathrm{F}}=268 \mathrm{~Hz}\right)[7$, starting material].

\section{Reaction of 2-(Difluoromethylsulfinyl)bromobenzene (6) with Benzene in the}

\section{Presence of Triflic Anhydride.}

To a solution of 6 (53 mg, $0.21 \mathrm{mmol}$ ) in benzene $(0.5 \mathrm{~mL})$ triflic anhydride (36 $\mu \mathrm{L}$, $0.21 \mathrm{mmol}$ ) was added at $5-10{ }^{\circ} \mathrm{C}$ under argon atmosphere. The reaction mixture was stirred overnight at rt, $\mathrm{CD}_{3} \mathrm{CN}$ was added to dissolved the formed precipitate and the solution was analyzed by ${ }^{19}$ F-NMR. Trifluoromethanesulfonic acid and trifluoromethanesulfonic acid difluoromethyl ester (10) were identified as major products. ${ }^{19} \mathrm{~F}$ NMR (benzene): triflic acid $(-78$, s), trifluoromethanesulfonic acid difluoromethyl ester -74.8 (t, $\left.J_{\mathrm{F}-\mathrm{F}}=3 \mathrm{~Hz}, 3 \mathrm{H}\right),-82.5\left(\mathrm{dq}, J_{\mathrm{F}-\mathrm{F}}=3 \mathrm{~Hz}, J_{\mathrm{F}-\mathrm{H}}=68 \mathrm{~Hz}, 2 \mathrm{H}\right)$. The following compounds were also identified in the reaction mixture based on ${ }^{19} \mathrm{~F}$ NMR: triflic anhydride (trace, -74, s), 2-(difluoromethylsulfinyl)brombenzene (m, -116- 123). The multiplet at (-97 - -98.5) probably belongs to $S$-(difluoromethyl)-phenyl-2bromophenylsulfonium cation (See spectrum). 
Preparation of Difluoromethyl Phenyl Sulfide. Thiophenol (42 g, $0.36 \mathrm{~mol})$ was dissolved in hexane (500 mL). Finely powdered $\mathrm{NaOH}$ ( 40 g, $1.0 \mathrm{~mol}$, powdered in glove box) and TDA (6 g, $0.0185 \mathrm{mmol}$ ) were added at room temperature under argon. The reaction mixture was warmed up to $65{ }^{\circ} \mathrm{C}$, a balloon was attached to the reaction vessel and $\mathrm{CF}_{2} \mathrm{HCl}$ gas was bubbled into the stirred mixture from a cylinder for $6 \mathrm{~h}$. The reaction was monitored by GC-MS. When all of the starting material was converted into the product, the mixture was cooled down to room temperature. The solid was filtered off and dissolved in sodium carbonate solution in water $(10 \%, 300 \mathrm{~mL})$. The resulting solution was extracted with hexane $(2 \times 300 \mathrm{~mL})$. The combined organic layer was extracted with sodium carbonate solution $(10 \%, 1 \times 200 \mathrm{~mL})$, water $(1 \times 200 \mathrm{~mL})$, brine $(1 \times 200 \mathrm{~mL})$ and was dried over anhydrous magnesium sulfate. The drying agent was filtered off and the solvent was removed in vacuum to give $53.7 \mathrm{~g}$ of the crude product (GC purity 93\%) as a colorless oil. Vacuum distillation of the crude product yielded 45.3 g of the expected product. Yield: 79\%. ${ }^{1} \mathrm{H}-\mathrm{NMR}\left(\mathrm{CDCl}_{3}\right): 6.84\left(\mathrm{t}, 1 \mathrm{H}, J_{\mathrm{H}-\mathrm{F}}=57.04 \mathrm{~Hz}\right)$, 7.36-7.48 (m, 3H), 7.56-7.64 (m, 2H). ${ }^{19} \mathrm{~F}-\mathrm{NMR}\left(\mathrm{CDCl}_{3}\right)$ : -91.88 (d, 2F, $\left.J_{\mathrm{H}-\mathrm{F}}=56.46 \mathrm{~Hz}\right)$. ${ }^{13} \mathrm{C}-\mathrm{NMR}\left(\mathrm{CDCl}_{3}\right): 121.2\left(\mathrm{t}, J_{\mathrm{C}-\mathrm{F}}=275.0 \mathrm{~Hz}\right), 126.3,129.5,129.9$, 135.5. GC-MS (EI, $m / z): 160\left(\mathrm{M}^{+}\right)$.

Preparation of Difluoromethyl Phenyl Sulfoxide (11). Difluoromethyl phenyl sulfide (44.0 g, $0.275 \mathrm{~mol})$ was dissolved in dichloromethane (300 mL). m-CPBA (55\%, $100 \mathrm{~g}$, $0.32 \mathrm{~mol})$ was added in DCM $(600 \mathrm{~mL})$ at $0-3^{\circ} \mathrm{C}$ over a period of $5 \mathrm{~h}$. The reaction mixture was extracted with sodium carbonate solution in water $(10 \%, 3 \times 300 \mathrm{~mL})$, with water $(1 \times 300 \mathrm{~mL})$ and with brine $(1 \times 300 \mathrm{~mL})$. The organic layer was dried over 
anhydrous magnesium sulfate and the drying agent was filtered off. The solvent was removed in vacuum to give $46.4 \mathrm{~g}$ of the crude product as a colorless oil (GC purity 91 \%). $10 \mathrm{~g}$ of this product was purified by gradient silica gel column chromatography (500 g silica gel, A eluent: $n$-hexane, B-eluent: ethyl-acetate, B was increased from 1 to $5 \%$ at a flow rate of $\left.0.25 \mathrm{ml} /\left(\min \times \mathrm{cm}^{-2}\right)\right)$ to give $8.3 \mathrm{~g}$ of the pure product. Yield: $80 \%$. ${ }^{1} \mathrm{H}$ NMR $\left(\mathrm{CDCl}_{3}\right): 6.04$ (t, 1H, $\left.J_{\mathrm{H}-\mathrm{F}}=55.11 \mathrm{~Hz}\right), 7.50-7.62(\mathrm{~m}, 3 \mathrm{H}), 7.65-7.73(\mathrm{~m}, 2 \mathrm{H}) .{ }^{19} \mathrm{~F}-$ $\operatorname{NMR}\left(\mathrm{CDCl}_{3}\right): 119.56\left(\mathrm{dd}, 1 \mathrm{~F}, J_{\mathrm{F}-\mathrm{F}}=261.78 \mathrm{~Hz}, J_{\mathrm{H}-\mathrm{F}}=55.11 \mathrm{~Hz}\right), 120.33\left(\mathrm{dd}, 1 \mathrm{~F}, J_{\mathrm{F}-\mathrm{F}}=\right.$ 261.78, $\left.J_{\mathrm{HF}}=55.11 \mathrm{~Hz}\right),{ }^{13} \mathrm{C}-\mathrm{NMR}\left(\mathrm{CDCl}_{3}\right): 120.9\left(\mathrm{t}, J_{\mathrm{C}-\mathrm{F}}=289.0 \mathrm{~Hz}\right), 125.5,129.6$, 132.9, 136.6. GC-MS (EI, m/z): $176\left(\mathrm{M}^{+}\right)$.

\section{Preparation of $S$-Difluoromethyl-S-phenyl-2,3,4,5-tetramethylphenylsulfonium}

Tetrafluoroborate (13). To a stirred solution of phenyl difluoromethyl sulfoxide (11) (4.00 g, $0.025 \mathrm{~mol})$ and 1,2,3,4-tetramethylbenzene (3.35 g, $0.025 \mathrm{~mol})$ in dry diethyl ether $(60 \mathrm{~mL})$ at $0{ }^{\circ} \mathrm{C}$ under argon was added trifluoromethanesulfonic acid anhydride ( $7.0 \mathrm{~g}, 0.025 \mathrm{~mol}$ ) in small portions over a period of 2 hours then the reaction mixture was stirred for 20 minutes at the same temperature. The formed oil was separated from the diethyl ether phase under nitrogen, then dry diethyl ether $(30 \mathrm{~mL})$ was added to the oil and the mixture was stirred again. The above mentioned procedure was repeated four times. The resulting oil was dissolved in dichloromethane $(50 \mathrm{~mL})$, the dichloromethane solution was extracted with sodium tetrafluoroborate solution in water (c = $1 \mathrm{M}, 5 \times 100$ $\mathrm{mL}$ ) and it was dried over anhydrous magnesium sulfate. The drying agent was filtered off and the dichloromethane was removed in vacuum. The product was obtained as a brown semisolid (5.9 g, 51\%). ${ }^{1} \mathrm{H}-\mathrm{NMR}\left(\mathrm{CDCl}_{3}\right)$ : 2.31 (s, 3H), 2.32 (s, 3H), 2.40 (s, 3H), 
2.57 (s, 3H), 7.49 (s, 1H), 7.65-7.95 (m, 5H), $8.12\left(\mathrm{t}, 1 \mathrm{H}, J_{\mathrm{H}-\mathrm{F}}=47.4 \mathrm{~Hz}\right) .{ }^{19} \mathrm{~F}-\mathrm{NMR}$ $\left(\mathrm{CDCl}_{3}\right):-99.87\left(\mathrm{dd}, 1 \mathrm{~F}, J_{\mathrm{F}-\mathrm{F}}=227.4 \mathrm{~Hz}, J_{\mathrm{H}-\mathrm{F}}=53.4 \mathrm{~Hz}\right),-100.58\left(\mathrm{dd}, 1 \mathrm{~F}, J_{\mathrm{F}-\mathrm{F}}=227.4\right.$ $\left.\mathrm{Hz}, J_{\mathrm{H}-\mathrm{F}}=53.40 \mathrm{~Hz}\right),-152.02(\mathrm{~s}, 1 \mathrm{~F}),-152.08$ (s, 3F). ${ }^{13} \mathrm{C}-\mathrm{NMR}\left(\mathrm{CDCl}_{3}\right):$ 17.0, 17.1, 18.0, 21.1, 113.0, 118.90 (t, $\left.J_{\mathrm{C}-\mathrm{F}}=297.5 \mathrm{~Hz}\right), 119.0,129.0,131.6,131.9,135.5,138.9$, 139.1, 140.0, 145.3. HRMS (FAB) calculated for $\mathrm{C}_{17} \mathrm{H}_{19} \mathrm{~F}_{2} \mathrm{~S}$. Expected: 293.1170. Found: 293.1170.

Preparation of Phenyl 2,3,4,5-Tetramethylphenyl Sulfide (15). To a solution of $S$ difluoromethyl-S-phenyl-2,3,4,5-tetramethylphenylsulfonium tetrafluoroborate (13, 380 $\mathrm{mg}, 1 \mathrm{mmol})$ in acetonitrile $(5 \mathrm{~mL})$ was added water $(1 \mathrm{~mL})$. The reaction mixture was stirred for 2 hours at room temperature then it was concentrated in vacuum. The precipitate was filtered off to give product $\mathbf{1 5}$ as a white solid (220 mg, $91 \%$ ). ${ }^{1} \mathrm{H}-\mathrm{NMR}$ $\left(\mathrm{CDCl}_{3}\right): 2.22$ (s, 3H), 2.24 (s, 3H), 2.25 (s, 3H), 2.36 (s, 3H), 7.04-7.14 (m, 3H), 7.167.25 (m, 3H). ${ }^{13} \mathrm{C}-\mathrm{NMR}\left(\mathrm{CDCl}_{3}\right): 16.5,17.1,18.0,20.8,125.4,127.7,128.8,129.1$, 134.2, 134.8, 136.4, 136.6, 137.4, 138.6. GC-MS (EI, m/z): $242\left(\mathrm{M}^{+}\right)$. HRMS calculated for $\mathrm{C}_{16} \mathrm{H}_{18} \mathrm{~S}$. Expected: 242.1129. Found: 242.1133.

\section{General Method for the Difluoromethylation of Aryl and Alkyl Sulfonic Acids. To}

the stirred solution of $\mathbf{1 3}(0.83 \mathrm{mmol})$ in dry acetonitrile $(0.5 \mathrm{~mL})$ was added the substrate (14 a-h) $(0.33 \mathrm{mmol})$ under $\mathrm{N}_{2}$. Detailed conditions are shown in Table 1. After the time showed in Table 1 known amount if 2,4,6-trifluoromesitilene or fluorobenzene was added as internal standard and the conversion was determined based on ${ }^{19} \mathrm{~F}-\mathrm{NMR}$. Dichloromethane $(10 \mathrm{~mL})$ was added and the diluted reaction mixture was extracted with 
sodium hydrogen carbonate solution $(10 \%, 2 \times 10 \mathrm{~mL})$. The solvent was evaporated in vacuum and the difluoromethyl ester was separated from 15 by gradient silica gel column chromatography. (Eluent: $n$-hexane, ethyl acetate).

Difluoromethyl 4-Methylbenzenesulfonate (16a). Yield: 77\%. Colorless oil. ${ }^{1} \mathrm{H}$ NMR $\left(\mathrm{CDCl}_{3}\right): 2.48(\mathrm{~s}, 3 \mathrm{H}), 6.78\left(\mathrm{t}, 1 \mathrm{H}, J_{\mathrm{H}-\mathrm{F}}=70.44 \mathrm{~Hz}\right), 7.35-7.45(\mathrm{~m}, 2 \mathrm{H}), 7.81-7.87$ (m, 2H). ${ }^{19} \mathrm{~F}-\mathrm{NMR}\left(\mathrm{CDCl}_{3}\right):-85.02\left(\mathrm{~d}, J_{\mathrm{H}-\mathrm{F}}=70.44 \mathrm{~Hz}\right) .{ }^{13} \mathrm{C}-\mathrm{NMR}\left(\mathrm{CDCl}_{3}\right): 21.9,114.1$ $\left(\mathrm{t}, J_{\mathrm{C}-\mathrm{F}}=266.5\right), 128.2,130.4,132.8,146.7 . \mathrm{MS}(\mathrm{EI}, \mathrm{m} / \mathrm{z}): 186\left(\mathrm{M}^{+}\right)$.

Difluoromethyl 4-Vinylbenzenesulfonate (16b). Yield: 67\%. Colorless oil. ${ }^{1} \mathrm{H}-\mathrm{NMR}$ $\left(\mathrm{CDCl}_{3}\right): 5.52(\mathrm{~d}, 1 \mathrm{H}, J=10.99 \mathrm{~Hz}), 5.95\left(\mathrm{dd}, 1 \mathrm{H},{ }^{3} J_{\mathrm{H}-\mathrm{H}}=17.58,{ }^{2} J_{\mathrm{H}-\mathrm{H}}=0.36 \mathrm{~Hz}\right), 6.77$ (dd, $\left.1 \mathrm{H},{ }^{3} J_{\mathrm{H}-\mathrm{H}}=10.99 \mathrm{~Hz},{ }^{3} J_{\mathrm{H}-\mathrm{H}}=17.58 \mathrm{~Hz}\right), 6.79\left(\mathrm{t}, 1 \mathrm{H}, J_{\mathrm{H}-\mathrm{F}}=71.15 \mathrm{~Hz}\right), 7.56-7.64(\mathrm{~m}$, 2H), 7.86-7.94 (m, 2H), ${ }^{19} \mathrm{~F}-\mathrm{NMR}\left(\mathrm{CDCl}_{3}\right)$ : -85.01 (d, $\left.J_{\mathrm{H}-\mathrm{F}}=71.15 \mathrm{~Hz}\right) .{ }^{13} \mathrm{C}-\mathrm{NMR}$ $\left(\mathrm{CDCl}_{3}\right): 114.1\left(\mathrm{t}, J_{\mathrm{C}-\mathrm{F}}=267.3 \mathrm{~Hz}\right), 119.2,127.3,128.5,134.3,135.0,144.4 . \mathrm{HRMS}$ calculated for $\mathrm{C}_{9} \mathrm{H}_{8} \mathrm{~F}_{2} \mathrm{O}_{3}$ S. Expected: 234.0162. Found: 234.0167.

Difluoromethyl 4-Hydroxybenzenesulfonate (16c). Yield: 84\%. Off-white semisolid. ${ }^{1} \mathrm{H}-\mathrm{NMR}\left(\mathrm{CDCl}_{3}\right)$ : 6.00-6.40 (br s, $\left.1 \mathrm{H}\right), 6.74$ (t, $\left.J_{\mathrm{H}-\mathrm{F}}=70.44 \mathrm{~Hz}, 1 \mathrm{H}\right), 6.95-7.05$ (m, 2H), 7.75-7.90 (m, 2H) ${ }^{19}$ F-NMR $\left(\mathrm{CDCl}_{3}\right)$ : -85.06 (d, $\left.J_{\mathrm{H}-\mathrm{F}}=70.44 \mathrm{~Hz}\right) .{ }^{13} \mathrm{C}-\mathrm{NMR}\left(\mathrm{CDCl}_{3}\right)$ : $113.9\left(\mathrm{t}, J_{\mathrm{C}-\mathrm{F}}=266.6 \mathrm{~Hz}\right), 116.4,126.8,130.8,161.6$. HRMS calculated for $\mathrm{C}_{7} \mathrm{H}_{6} \mathrm{~F}_{2} \mathrm{O}_{4} \mathrm{~S}$. Expected: 223.9955. Found: 223.9945. 
Difluoromethyl 2,4,6-Trimethylbenzenesulfonate (16d). Yield: 78\%. Colorless oil. ${ }^{1} \mathrm{H}-\mathrm{NMR}\left(\mathrm{CDCl}_{3}\right)$ : 2.34 (s, 3H), 2.64 (s, 6H), 6.70 (t, 1H, $\left.J_{\mathrm{H}-\mathrm{F}}=70.96\right), 6.99(\mathrm{~s}, 1 \mathrm{H}) .{ }^{19} \mathrm{~F}-$ NMR -(CDCl $)$ ):-85.38 (d, $\left.J_{\mathrm{H}-\mathrm{F}}=70.56 \mathrm{~Hz}\right) .{ }^{13} \mathrm{C}-\mathrm{NMR}\left(\mathrm{CDCl}_{3}\right)$ : 21.3, 22.7, 113.9 (t, $J_{\mathrm{C}-\mathrm{F}}$ = $265.9 \mathrm{~Hz}), 130.9,132.2,140.3,145.0$. HRMS calculated for $\mathrm{C}_{10} \mathrm{H}_{12} \mathrm{~F}_{2} \mathrm{O}_{3} \mathrm{~S}$. Expected: 250.0475. Found: 252.0476.

Bis(difluoromethyl) o-benzenedisulfonate (16e) Yield: 89\%. Colorless oil. ${ }^{1} \mathrm{H}-\mathrm{NMR}$ $\left(\mathrm{CDCl}_{3}\right)$ : $6.97\left(\mathrm{t}, 2 \mathrm{H}, J_{\mathrm{H}-\mathrm{F}}=70.04 \mathrm{~Hz}\right), 7.90-8.10(\mathrm{~m}, 2 \mathrm{H}), 8.30-8.50(\mathrm{~m}, 2 \mathrm{H}) .{ }^{19} \mathrm{~F}-\mathrm{NMR}$ $\left(\mathrm{CDCl}_{3}\right):-84.66\left(\mathrm{~d}, J_{\mathrm{H}-\mathrm{F}}=70.04 \mathrm{~Hz}\right) .{ }^{13} \mathrm{C}-\mathrm{NMR}\left(\mathrm{CDCl}_{3}\right): 114.4\left(\mathrm{t}, J_{\mathrm{C}-\mathrm{F}}=270.8 \mathrm{~Hz}\right)$, 133.5, 134.8, 135.7. HRMS calculated for $\mathrm{C}_{8} \mathrm{H}_{6} \mathrm{~F}_{4} \mathrm{O}_{6} \mathrm{~S}_{2}$. Expected: 337.9542. Found: 337.9544.

Difluoromethyl 3-nitrobenzenesulfonate (16f) Yield: 74\%. Yellow oil. ${ }^{1} \mathrm{H}-\mathrm{NMR}$ $\left(\mathrm{CDCl}_{3}\right): 6.84\left(\mathrm{t}, 1 \mathrm{H}, J_{\mathrm{H}-\mathrm{F}}=70.18 \mathrm{~Hz}\right), 7.85$ (t, $\left.1 \mathrm{H}, J=8.07 \mathrm{~Hz}\right), 8.24-8.30(\mathrm{~m}, 1 \mathrm{H}), 8.54-$ 8.60 (m, 1H), 8.74-8.80 (m, 1H). ). ${ }^{19} \mathrm{~F}-\mathrm{NMR}\left(\mathrm{CDCl}_{3}\right)$ : -84.68 (d, $\left.J_{\mathrm{H}-\mathrm{F}}=70.18 \mathrm{~Hz}\right) .{ }^{13} \mathrm{C}-$ NMR $\left(\mathrm{CDCl}_{3}\right): 114.1\left(\mathrm{t}, J_{\mathrm{C}-\mathrm{F}}=270.1 \mathrm{~Hz}\right), 123.4,129.5,131.3,133.4,137.8,148.4 . \mathrm{MS}$ (EI, $m / z): 217\left(\mathrm{M}^{+}\right)$.

Difluoromethyl 2-naphthalenesulfonate (16g). Yield: 54\%. Colorless oil. ${ }^{1} \mathrm{H}-\mathrm{NMR}$ $\left(\mathrm{CDCl}_{3}\right): 6.85\left(\mathrm{t}, 1 \mathrm{H}, J_{\mathrm{H}-\mathrm{F}}=70.50 \mathrm{~Hz}\right), 7.60-7.80(\mathrm{~m}, 2 \mathrm{H}), 7.8-8.2(\mathrm{~m}, 4 \mathrm{H}), 8.55(\mathrm{~s}, 1 \mathrm{H})$. ${ }^{19}$ F-NMR $\left(\mathrm{CDCl}_{3}\right):-84.78\left(\mathrm{~d}, J_{\mathrm{H}-\mathrm{F}}=70.19 \mathrm{~Hz}\right) .{ }^{13} \mathrm{C}-\mathrm{NMR}\left(\mathrm{CDCl}_{3}\right): 114.2\left(\mathrm{t}, J_{\mathrm{C}-\mathrm{F}}=267.4\right)$, 122.2, 128.3, 128.4, 129.8, 130.3, 130.4, 130.5, 132.0, 132.6, 136.0. HRMS calculated for $\mathrm{C}_{11} \mathrm{H}_{8} \mathrm{~F}_{2} \mathrm{O}_{3} \mathrm{~S}$. Expected: 258.0162. Found: 258.0167. 
Difluoromethyl 10-camphorsulfonate (16h). Yield: 86\%. Colorless oil. ${ }^{1} \mathrm{H}$-NMR ( $\left.\mathrm{CDCl}_{3}\right): 0.88$ (s, 3H), 1.09 (s, 3H), 1.40-1.50 (m, 1H), 1.67-1.78 (m, 1H), 1.96 (d, $1 \mathrm{H}, J$ $=19 \mathrm{~Hz}), 2.00-2.18(\mathrm{~m}, 2 \mathrm{H}), 2.30-2.45(\mathrm{~m}, 2 \mathrm{H}), 3.23(\mathrm{~d}, 1 \mathrm{H}, J=15 \mathrm{~Hz}), 3.75$ (d, $1 \mathrm{H}, J=$ $15 \mathrm{~Hz}), 6.81\left(\mathrm{t}, 1 \mathrm{H}, J_{\mathrm{F}-\mathrm{H}}=70.53 \mathrm{~Hz}\right) \cdot{ }^{19} \mathrm{~F}-\mathrm{NMR}\left(\mathrm{CDCl}_{3}\right):-84.78\left(\mathrm{dd}, J_{\mathrm{F}-\mathrm{F}}=225.83 \mathrm{~Hz}, J_{\mathrm{F}-}\right.$ $\left.{ }_{\mathrm{H}}=70.53 \mathrm{~Hz}\right),-85.23\left(\mathrm{dd}, J_{\mathrm{F}-\mathrm{F}}=225.83 \mathrm{~Hz}, J_{\mathrm{F}-\mathrm{H}}=70.53 \mathrm{~Hz}\right) .{ }^{13} \mathrm{C}-\mathrm{NMR}\left(\mathrm{CDCl}_{3}\right): 19.8$, 19.9, 25.4, 27.0, 42.5, 43.0, 48.3, 51.2, 58.4, $114.2\left(\mathrm{t}, J_{\mathrm{C}-\mathrm{F}}=267.4 \mathrm{~Hz}\right), 213.5 . \mathrm{HRMS}$ calculated for $\mathrm{C}_{11} \mathrm{H}_{16} \mathrm{~F}_{2} \mathrm{O}_{4} \mathrm{~S}$. Expected: 282.0737. Found: 282.0735.

$N$-(Difluoromethyl)triethylammonium Tetrafluoroborate (17). To a solution of triethyl amine (101 mg, $1 \mathrm{mmol})$ in acetonitrile $(1 \mathrm{~mL}) \mathrm{S}$-(difluoromethyl)-S-phenyl2,3,4,5-tetramethylphenylsulfonium tetrafluoroborate (13) $(400 \mathrm{mg}, 1.05 \mathrm{mmol})$ in acetonitrile (1mL) was added. The mixture was stirred overnight, followed by evaporation of the solvent in vacuum. The remaining solid was stirred with diethyl ether $(5 \mathrm{~mL})$ then the solid was filtered off. The stirring and filtration was repeated three times to give $167 \mathrm{mg}(70 \%)$ of the crude product as a yellowish solid. The product was dissolved in DCM, morpholinomethyl polystyrene $\mathrm{2}^{2}(700 \mathrm{mg}$, loading: $4.2 \mathrm{mmol} / \mathrm{g})$ was added and the mixture was stirred for $30 \mathrm{~min}$. The resin was filtered off and it washed with DCM. The DCM was evaporated in vacuum to give $143 \mathrm{mg}$ of the pure product as a yellowish semi solid. $\mathrm{mp}=239-242{ }^{\circ} \mathrm{C}\left(\mathrm{dec}\right.$.). ${ }^{1} \mathrm{H}-\mathrm{NMR}\left(\mathrm{DMSO}-d_{6}\right): 1.25$ (q, $J=7.32$ Hz, 9H), 3.55 (t, $J=7.29 \mathrm{~Hz}, 6 \mathrm{H}$ ), 7.32 (t, $\left.J_{\mathrm{H}-\mathrm{F}}=57.65 \mathrm{~Hz}, 1 \mathrm{H}\right) .{ }^{19}$ F-NMR (DMSO-d 6 ): 112.00 (d, $\left.J_{\mathrm{H}-\mathrm{F}}=57.65 \mathrm{~Hz}, 2 \mathrm{~F}\right),-147.64$ (s, 1F), -147.70 (s, 3F). ${ }^{13} \mathrm{C}-\mathrm{NMR}$ (DMSO-d 6 ):

\footnotetext{
${ }^{2}$ Novabiochem, cat numb: 01-64-0171
} 
7.8, 51.5, 115.0 (t, $J_{\mathrm{C}-\mathrm{F}}=273.1 \mathrm{~Hz}$ ). HRMS calculated for $\mathrm{C}_{7} \mathrm{H}_{16} \mathrm{~F}_{2} \mathrm{~N}$. Expected: 152.1251. Found: 152.1248.

$N$-(Difluoromethyl)- $N$, $N$-dimethylanilinium Tetrafluoroborate (18)

Yield $=63 \% .{ }^{1} \mathrm{H} \mathrm{NMR}\left(\mathrm{CDCl}_{3} / \mathrm{DMSO}-d_{6}\right): 3.82(\mathrm{~s}, 6 \mathrm{H}), 7.60\left(t, J_{\mathrm{H}-\mathrm{F}}=58.6 \mathrm{~Hz}, 1 \mathrm{H}\right)$, $7.73(m, 3 \mathrm{H}), 8.02(m, 2 \mathrm{H}) .{ }^{19} \mathrm{~F}$ NMR $\left(\mathrm{CDCl}_{3} / \mathrm{DMSO}-d_{6}\right):-110.7\left(d, J_{\mathrm{H}-\mathrm{F}}=58.6 \mathrm{~Hz}\right.$, 2F),-147.63(s, 1F), -147.69(s, 3F). ${ }^{13} \mathrm{C}$ NMR (DMSO- $\left.d_{6}\right): 47.9,114.7\left(t, J_{C-F}=276.9 \mathrm{~Hz}\right)$, 122.6, 130.2, 131.3, 139.3. HRMS calculated for $\mathrm{C}_{9} \mathrm{H}_{12} \mathrm{~F}_{2} \mathrm{~N}$ Expected: 172.0938. Found: 172.0943.

\section{$N$-(Difluoromethyl)- $N$, $N$-dimethyl- 4-methylanilinium Tetrafluoroborate (19)}

Yield $=60 \% .{ }^{1} \mathrm{H}$ NMR $\left(\mathrm{CDCl}_{3} / \mathrm{DMSO}-d_{6}\right): 2.43(\mathrm{~s}, 3 \mathrm{H}) \cdot 3.78(\mathrm{~s}, 6 \mathrm{H}), 7.37\left(\mathrm{t}, J_{\mathrm{H}-\mathrm{F}}=59.2\right.$ $\mathrm{Hz}, 1 \mathrm{H}), 7.44(\mathrm{~m}, 2 \mathrm{H}), 7.68(\mathrm{~m}, 2 \mathrm{H}) .{ }^{19} \mathrm{~F}$ NMR $\left(\mathrm{CDCl}_{3} / \mathrm{DMSO}-d_{6}\right):-111.44\left(d, J_{\mathrm{H}-\mathrm{F}}=59.2\right.$ Hz, 2H), -151.01(s, 1H), -151.07 (s, 1H). ${ }^{13} \mathrm{C}$ NMR (CDCl $/$ DMSO- $\left._{6}\right): 20.6,48.6,115$. $02\left(t, J_{C-F}=279.5 \mathrm{~Hz}\right), 121.3,131.2,136.6,142.4$. HRMS calculated for $\mathrm{C}_{10} \mathrm{H}_{14} \mathrm{~F}_{2} \mathrm{~N}$. Expected: 186.1094., Found: 186.1096.

N,N'-Bis(difluoromethyl)imidazolium Tetrafluoroborate Hemihydrate (23). To a solution of imidazole (34 mg, $0.5 \mathrm{mmol}$ ) in a mixture of $\mathrm{MeCN}(2 \mathrm{~mL})$ and $\mathrm{CH}_{2} \mathrm{Cl}_{2}(2$ $\mathrm{mL}$ ) a solution of $S$-difluoromethyl-S-phenyl-2,3,4,5-tetramethylphenylsulfonium tetrafluoroborate (13) $(400 \mathrm{mg}, 1.05 \mathrm{mmol})$ in acetonitrile $(1.0 \mathrm{~mL})$ was added under argon atmosphere at room temperature. The reaction mixture was stirred for one hour then $\mathrm{K}_{2} \mathrm{CO}_{3}(80 \mathrm{mg}, 0.58 \mathrm{mmol})$ was added. The reaction mixture was stirred overnight, the precipitate was filtered off. The solvent was removed in vacuum and the remaining semi solid was stirred with diethyl ether. The solution was decanted, the remaining semi 
solid was washed with diethyl ether and the solvent was decanted again. The remaining semisolid was dried in vacuum to give $108 \mathrm{mg}$ of the expected product. Yield: $84 \% .{ }^{1} \mathrm{H}$ NMR (DMSO- $d_{6}$ ): 3.37 (br s, $\mathrm{H}_{2} \mathrm{O}, 1 \mathrm{H}$ ), 8.07 (t, $\left.J_{\mathrm{H}-\mathrm{F}}=58.87 \mathrm{~Hz}, 2 \mathrm{H}\right), 8.51(\mathrm{~s}, 2 \mathrm{H}), 10.34$ (s, 1H). ${ }^{19}$ F-NMR (DMSO- $d_{6}$ ): -96.15 (d, 4F, $J_{H-F}=58.87 \mathrm{~Hz}$ ), -147.69 (s, 1F), -147.73 (s, 3F). ${ }^{13} \mathrm{C}-\mathrm{NMR}$ (DMSO- $d_{6}$ ): 108.9 (t, $J_{\mathrm{C}-\mathrm{F}}=259.0 \mathrm{~Hz}$ ), 120.0, 138.0. HRMS calculated for $\mathrm{C}_{5} \mathrm{H}_{5} \mathrm{~F}_{2} \mathrm{~N}_{4}$. Expected: 169.0389. Found: 169.0383 .

$N$-Difluoromethyl- $N$ '-methylimidazolium Tetrafluoroborate (24). To a solution of $N$ methylimidazole (20) (41 mg, $0.5 \mathrm{mmol})$ in acetonitrile $(1 \mathrm{~mL})$ a solution of $S$ difluoromethyl-S-phenyl-2,3,4,5-tetramethylphenylsulfonium tetrafluoroborate (200 mg, $0.54 \mathrm{mmol})$ in acetonitrile $(2 \mathrm{~mL})$ was added dropwise under argon atmosphere at room temperature. The reaction mixture was stirred for 6 hours, followed by evaporation of the solvent in vacuum. The remaining semi solid was stirred with diethyl ether and the ether was decanted. The stirring and decantation were repeated three times to give $71 \mathrm{mg}$ (77 \%) of the expected product as a yellowish semi solid. ${ }^{1} \mathrm{H}-\mathrm{NMR}$ (DMSO- $\mathrm{d}_{6}$ ): 3.90 (s, 3H), $7.90(\mathrm{~s}, 1 \mathrm{H}), 7.98\left(\mathrm{t}, J_{\mathrm{H}-\mathrm{F}}=59.0 \mathrm{~Hz}, 1 \mathrm{H}\right), 8.20(\mathrm{~s}, 1 \mathrm{H}), 9.66(\mathrm{~s}, 1 \mathrm{H}) \cdot{ }^{19} \mathrm{~F}-\mathrm{NMR}$ (DMSO-d $d_{6}$ ): -94.84 (d, $\left.J_{\mathrm{H}-\mathrm{F}}=59.0,2 \mathrm{~F}\right),-147.63$ (s, 1F), -147.70 (s, 3F). ${ }^{13} \mathrm{C}-\mathrm{NMR}$ (DMSO- $d_{6}$ ): 36.6, 108.7 (t, $J_{\mathrm{C}-\mathrm{F}}=256.0 \mathrm{~Hz}$ ), 118.6, 125.2, 136.9. HRMS calculated for $\mathrm{C}_{5} \mathrm{H}_{7} \mathrm{~F}_{2} \mathrm{~N}_{2}$. Expected: 133.0577. Found: 133.0574 .

1-Difluoromethyl-2,3-dimethylimidazolium Tetrafluoroborate (25). To a solution of 1,2-dimethylimidazole (21) $(77 \mathrm{mg}, 0.79 \mathrm{mmol})$ in acetonitrile $(1 \mathrm{~mL})$ a solution of $S$ difluoromethyl-S-phenyl-2,3,4,5-tetramethylphenylsulfonium tetrafluoroborate (13) (310 
mg, $0.82 \mathrm{mmol}$ ) in acetonitrile was added drop wise under argon atmosphere at room temperature. The reaction mixture was stirred overnight followed by evaporation of the solvent in vacuum. The remaining solid was stirred with diethyl ether, then the solid was filtered off. The stirring and filtration were repeated three times to give $126 \mathrm{mg}$ (81\%) of the expected product as a white solid. $\mathrm{mp}=248-250{ }^{\circ} \mathrm{C}$ (dec.). ${ }^{1} \mathrm{H}-\mathrm{NMR}\left(\right.$ DMSO- $\left.\mathrm{d}_{6}\right)$ : $2.75(\mathrm{~s}, 3 \mathrm{H}), 3.81(\mathrm{~s}, 3 \mathrm{H}), 7.83\left(\mathrm{~d}, J_{\mathrm{H}-\mathrm{F}}=2.29 \mathrm{~Hz}, 1 \mathrm{H}\right), 8.07\left(\mathrm{t}, J_{\mathrm{H}-\mathrm{F}}=56.93 \mathrm{~Hz}, 1 \mathrm{H}\right), 8.09$ (d, $J=2.29 \mathrm{~Hz}, 1 \mathrm{H}) .{ }^{19} \mathrm{~F}-\mathrm{NMR}\left(\mathrm{DMSO}-d_{6}\right):-95.95\left(\mathrm{~d}, J_{\mathrm{H}-\mathrm{F}}=58.65 \mathrm{~Hz}, 2 \mathrm{~F}\right),-147.65$ (s, 1F), -147.71 (s, 3F). ${ }^{13} \mathrm{C}-\mathrm{NMR}$ (DMSO- $\left.d_{6}\right): 10.2,35.0,108.5$ (t, $J_{\mathrm{C}-\mathrm{F}}=254.1 \mathrm{~Hz}$ ), 116.9, 124.1, 146.3. HRMS calculated for $\mathrm{C}_{6} \mathrm{H}_{9} \mathrm{~F}_{2} \mathrm{~N}_{2}$. Expected: 147.0734. Found: 147.0741.

P-(Difluoromethyl)triphenylphosphonium Tetrafluoroborate (26). A mixture of triphenylphosphine $\quad(131 \quad \mathrm{mg}, \quad 0.5 \mathrm{mmol}), \quad$ S-difluoromethyl-S-phenyl-2,3,4,5tetramethylphenylsulfonium tetrafluoroborate (200 mg, $0.53 \mathrm{mmol})$ and DIAD (15 mg, $0.074 \mathrm{mmol})$ in dichloromethane $(1 \mathrm{~mL})$ was stirred at rt overnight. Diethyl ether was added to the reaction mixture. The resulting precipitate was filtered off followed by stirring with diethyl ether again. The solid was filtered off to give $166 \mathrm{mg}$ of the expected product as a white crystal. Yield: 83\%. mp.: $130-132{ }^{\circ} \mathrm{C}$ (dec.). ${ }^{1} \mathrm{H}-\mathrm{NMR}$ (DMSO- $\mathrm{d}_{6}$ ): 7.80-7.95 (m, 12H), 8.02-8.14 (m, 3H), $8.41\left(\mathrm{dt}, 1 \mathrm{H}, J_{\mathrm{H}-\mathrm{F}}=47.10 \mathrm{~Hz}, J_{\mathrm{H}-\mathrm{P}}=29.30 \mathrm{~Hz}\right)$. ${ }^{31} \mathrm{P}\left(\mathrm{DMSO}-d_{6}\right): 19.88\left(\mathrm{t}, J_{\mathrm{F}-\mathrm{P}}=77.28 \mathrm{~Hz}\right),{ }^{19} \mathrm{~F}-\mathrm{NMR}\left(\mathrm{DMSO}-d_{6}\right)$ : $-125.74\left(\mathrm{dd}, J_{\mathrm{F}-\mathrm{P}}=\right.$ $\left.77.28 \mathrm{~Hz}, J_{\mathrm{H}-\mathrm{F}}=47.10 \mathrm{~Hz}, 2 \mathrm{~F}\right),-147.65$ (s, 1F), -147.70 (s, 3F); ${ }^{13} \mathrm{C}-\mathrm{NMR}\left(\mathrm{DMSO}-d_{6}\right.$ ): $111.9\left(\mathrm{t}, J_{\mathrm{C}-\mathrm{P}}=84.7 \mathrm{~Hz}\right) ; 114.3\left(\mathrm{dt}, J_{\mathrm{C}-\mathrm{F}}=268.6 \mathrm{~Hz}, J_{\mathrm{C}-\mathrm{P}}=83.9 \mathrm{~Hz}\right), 131.0\left(\mathrm{~d}, J_{\mathrm{C}-\mathrm{P}}=13.0\right.$ $\mathrm{Hz}), 134.8\left(\mathrm{~d}, J_{\mathrm{C}-\mathrm{P}}=10.7 \mathrm{~Hz}\right), 136.8\left(\mathrm{~d}, J_{\mathrm{C}-\mathrm{P}}=3.0 \mathrm{~Hz}\right)$. HRMS calculated for $\mathrm{C}_{19} \mathrm{H}_{16} \mathrm{~F}_{2} \mathrm{P}$. Expected: 313.0958. Found: 313.0958. 


\section{P- (Difluoromethyl)tris(4-flurophenyl)phosphonium Tetrafluoroborate (27)}

Yield $=75 \% .{ }^{1} \mathrm{H}$ NMR $\left(\mathrm{CDCl}_{3} / \mathrm{DMSO}_{-}\right): 7.48(\mathrm{~m}, 6 \mathrm{H}), 7.82(\mathrm{~m}, 6 \mathrm{H}), 7.91\left(\mathrm{dt}, J_{H-F}=\right.$ $\left.47.5 \mathrm{~Hz}, J_{H-P}=31.4 \mathrm{~Hz}, 1 \mathrm{H}\right) .{ }^{19} \mathrm{~F}$ NMR $\left(\mathrm{CDCl}_{3} / \mathrm{DMSO}_{-} d_{6}\right):-96.4(\mathrm{~s}, 3 \mathrm{~F}),-126.28\left(d d, J_{\mathrm{F}-}\right.$ $\left.\mathrm{P}=80.6 \mathrm{~Hz}, \quad J_{\mathrm{H}-\mathrm{F}}=47.6 \mathrm{~Hz}, 2 \mathrm{~F}\right),-152.01(\mathrm{~s}, 1 \mathrm{~F}),-152.06(\mathrm{~s}, 3 \mathrm{~F}) . \quad{ }^{13} \mathrm{C} \mathrm{NMR}$ $\left.\left(\mathrm{CDCl}_{3} / \mathrm{DMSO}_{-}\right)_{6}\right): 107.4(d, \mathrm{~J}=90.1 \mathrm{~Hz}), 113.7\left(d t, J_{\mathrm{C}-\mathrm{F}}=269.6 \mathrm{~Hz}, \mathrm{~J}_{\mathrm{C}-\mathrm{p}}=88.2 \mathrm{~Hz}\right)$, 119.1(m), 137.5(m), 167.8(d, $\left.J_{\mathrm{C}-\mathrm{F}}=263.7 \mathrm{~Hz}\right) .{ }^{31} \mathrm{P} \mathrm{NMR}\left(\mathrm{CDCl}_{3} / \mathrm{DMSO}-d_{6}\right): 19.03\left(t,{ }^{2} J_{P-}\right.$ $F^{=} 80.8 \mathrm{~Hz}$ ). HRMS calculated for $\mathrm{C}_{19} \mathrm{H}_{13} \mathrm{~F}_{5} \mathrm{P}$. Expected: 367.0695 . Found: 367.0669 .

\section{P- (Difluoromethyl)tricyclohexylphosphonium Tetrafluoroborate (28)}

Yield $=73 \% .{ }^{1} \mathrm{H}$ NMR $\left(\mathrm{CDCl}_{3} / \mathrm{DMSO}_{-} \mathrm{d}_{6}\right): 1.31-2.13(\mathrm{~m}, 30 \mathrm{H}), 2.80(\mathrm{~m}, 3 \mathrm{H}), 7.28\left(\mathrm{dt}, J_{H-}\right.$ $\left.F=46.8 \mathrm{~Hz}, J_{H-P}=26.3 \mathrm{~Hz}, 1 \mathrm{H}\right) .{ }^{19} \mathrm{~F} \mathrm{NMR}\left(\mathrm{CDCl}_{3} / \mathrm{DMSO}-d_{6}\right):-123.39\left(d d, J_{\mathrm{F}-\mathrm{P}}=59.4 \mathrm{~Hz}\right.$, $\left.J_{\mathrm{H}-\mathrm{F}}=46.7 \mathrm{~Hz}, 2 \mathrm{~F}\right),-152.07(\mathrm{~s}, 1 \mathrm{~F}),-152.12(\mathrm{~s}, 3 \mathrm{~F}) .{ }^{13} \mathrm{C} \mathrm{NMR}\left(\mathrm{CDCl}_{3} / \mathrm{DMSO}_{6}\right): 24.9$, 26.0, 26.4, 29.3(d, $J=32.3 \mathrm{~Hz}), 113.7\left(d t, J_{\mathrm{C}-\mathrm{F}}=267.0 \mathrm{~Hz}, \mathrm{~J}_{\mathrm{C}-\mathrm{P}}=67 \mathrm{~Hz}\right), 119.1(\mathrm{~m})$, 137.5(m), 167.8(d, $\left.J_{\mathrm{C}-\mathrm{F}}=263.7 \mathrm{~Hz}\right) .{ }^{31} \mathrm{P} \mathrm{NMR}\left(\mathrm{CDCl}_{3} / \mathrm{DMSO}_{6}\right): 32.46\left(t,{ }^{2} J_{P-F}=59.4\right.$ Hz). HRMS calculated for $\mathrm{C}_{19} \mathrm{H}_{34} \mathrm{~F}_{2} \mathrm{P}$. Expected: 331.2366. Found: 331.2361. 


\section{New Electrophilic Difluoromethylating Reagent}

G. K. Surya Prakash,* Csaba Weber, Sujith Chacko and George A. Olah*

Loker Hydrocarbon Research Institute and Department of Chemistry, University of Southern California, Los Angeles, California 900891661

gprakash@usc.edu; olah@usc.edu

Supporting Information

Spectra 


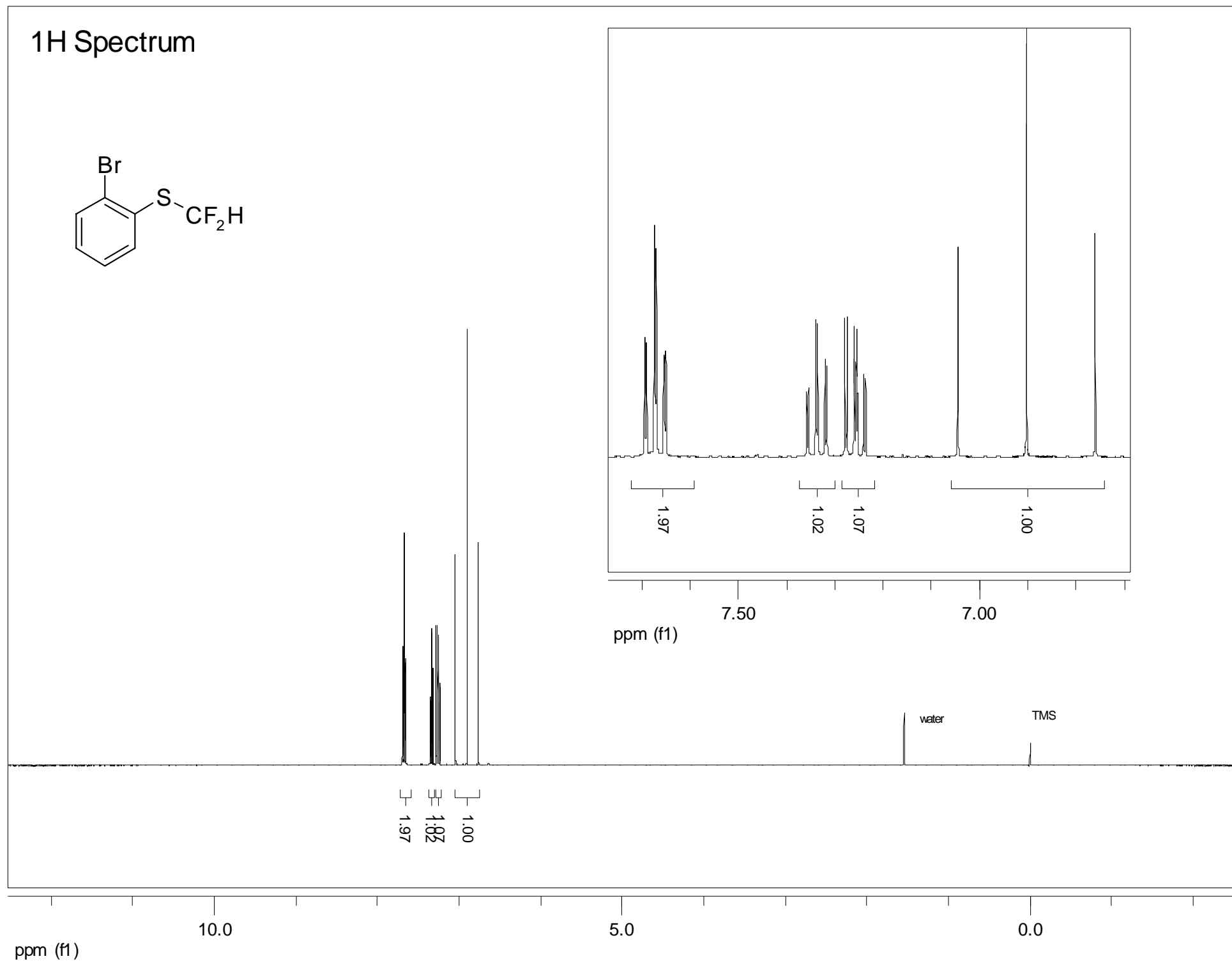

- S18 - 


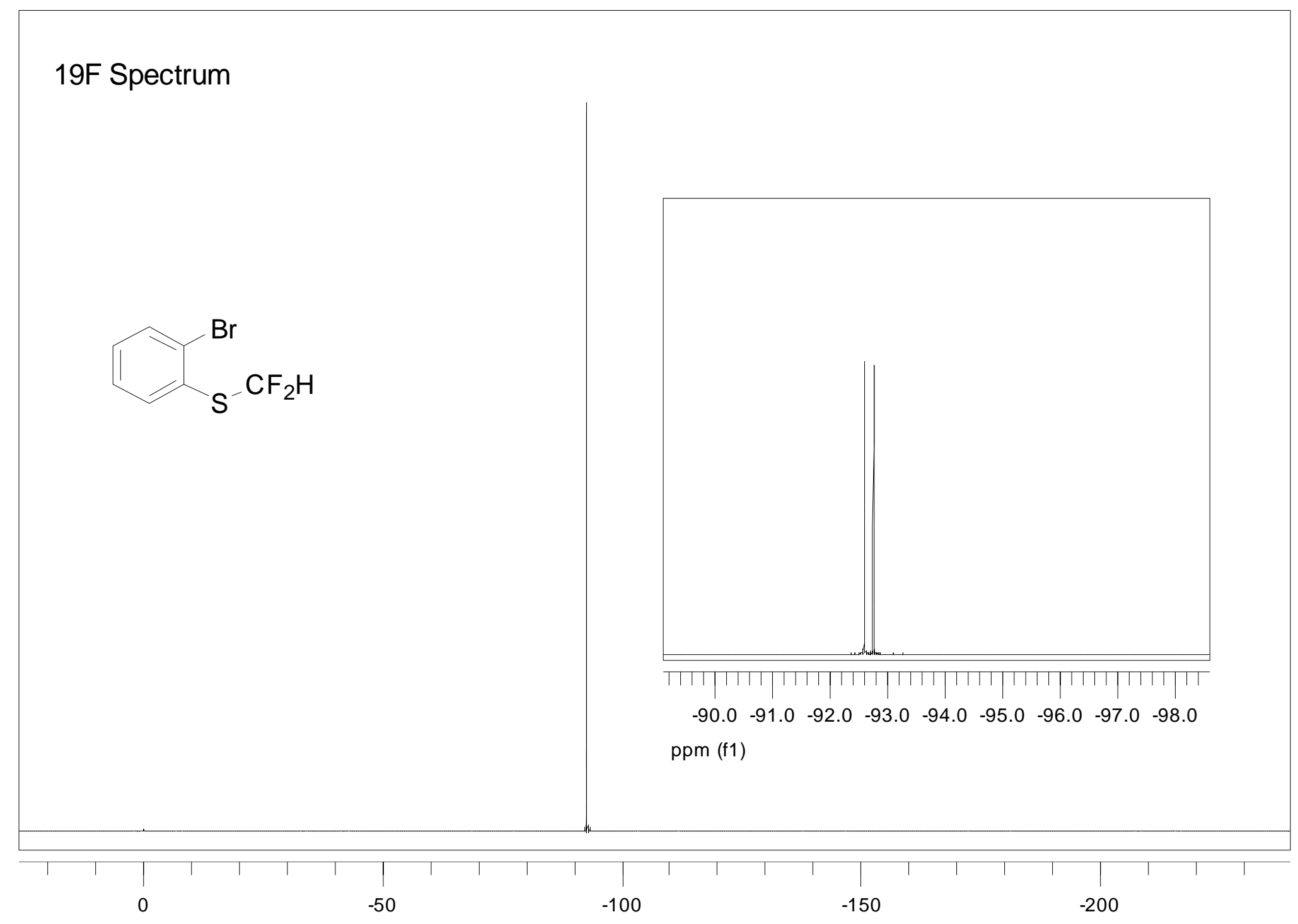

ppm (f1) 


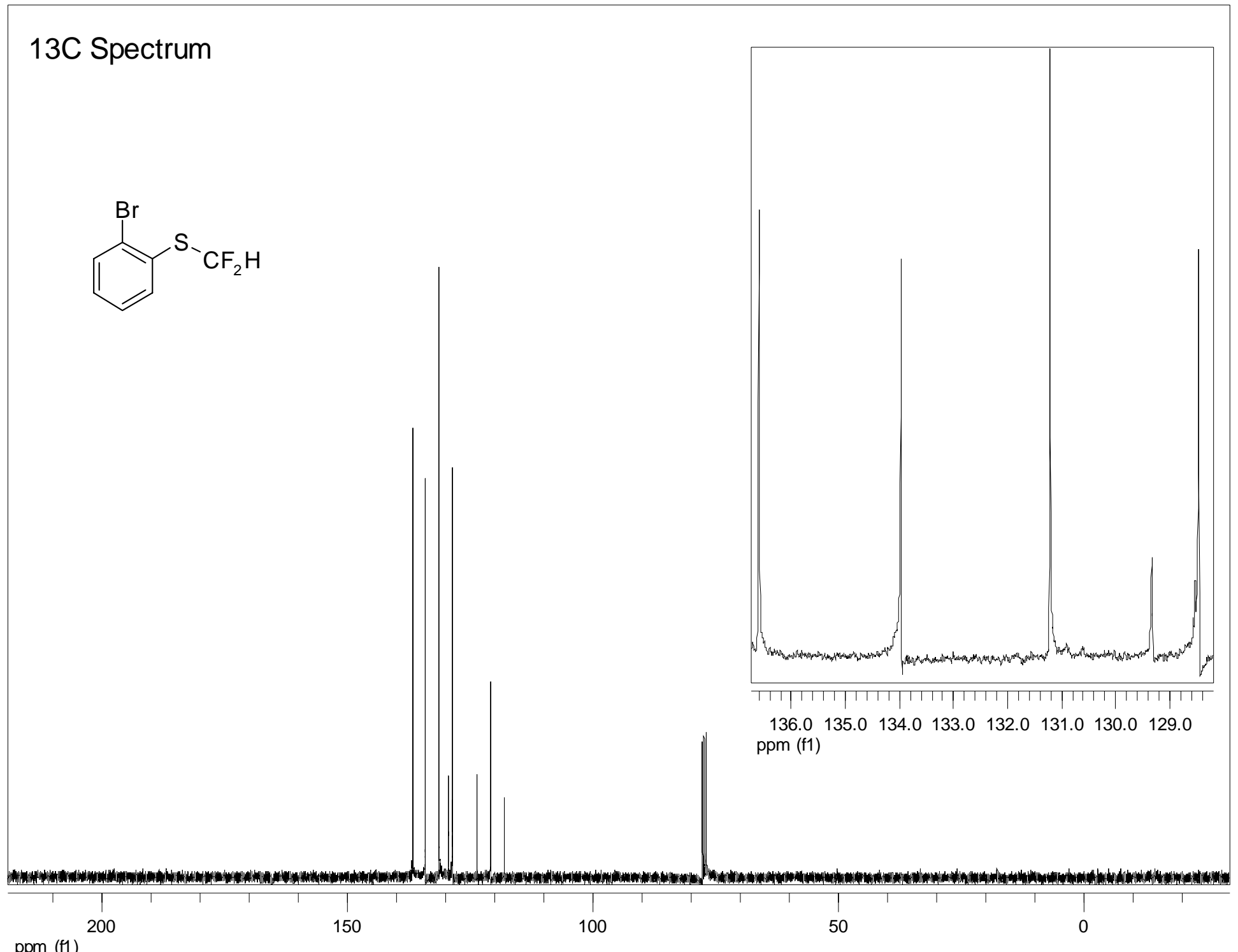

ppm (f1) 


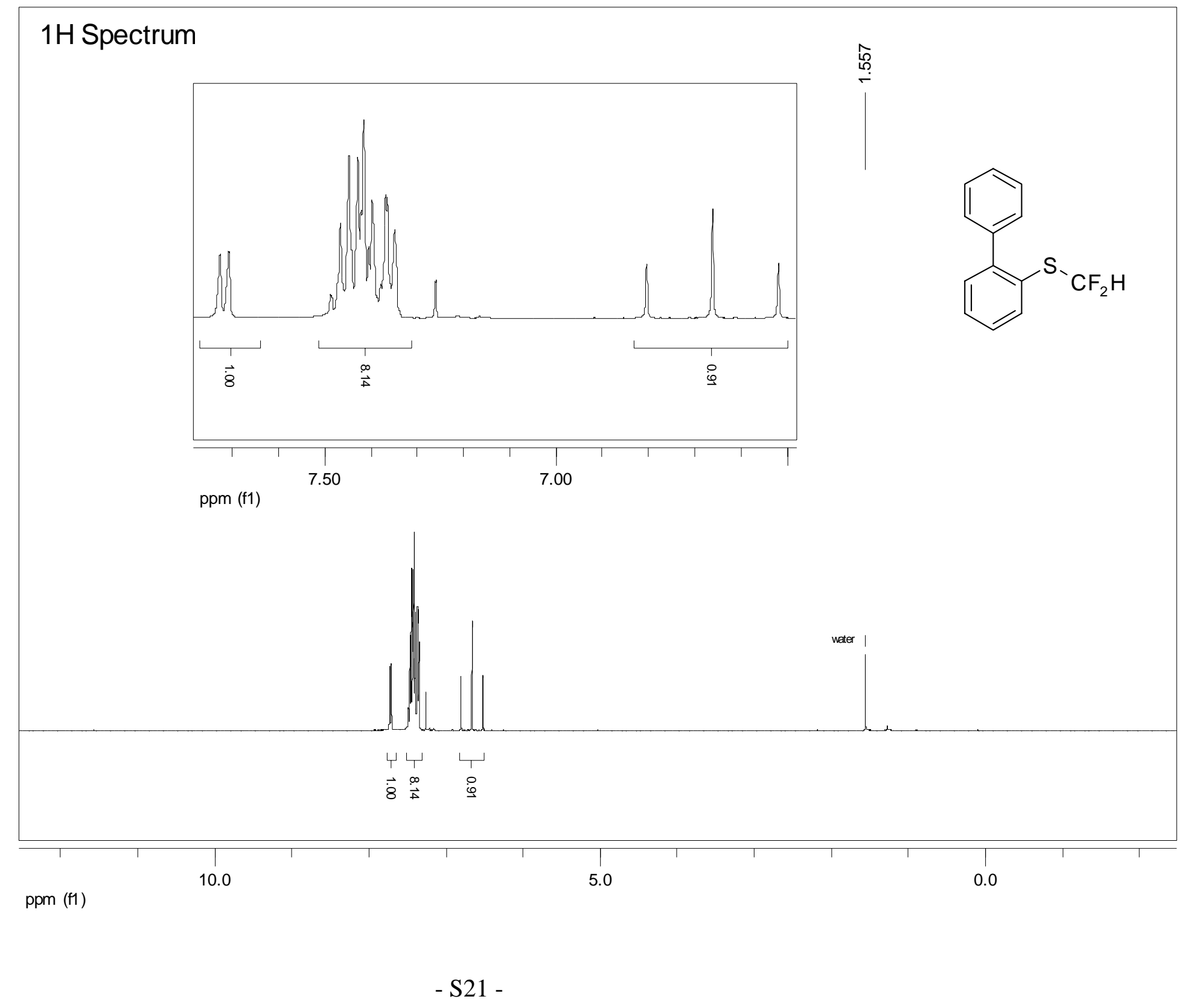




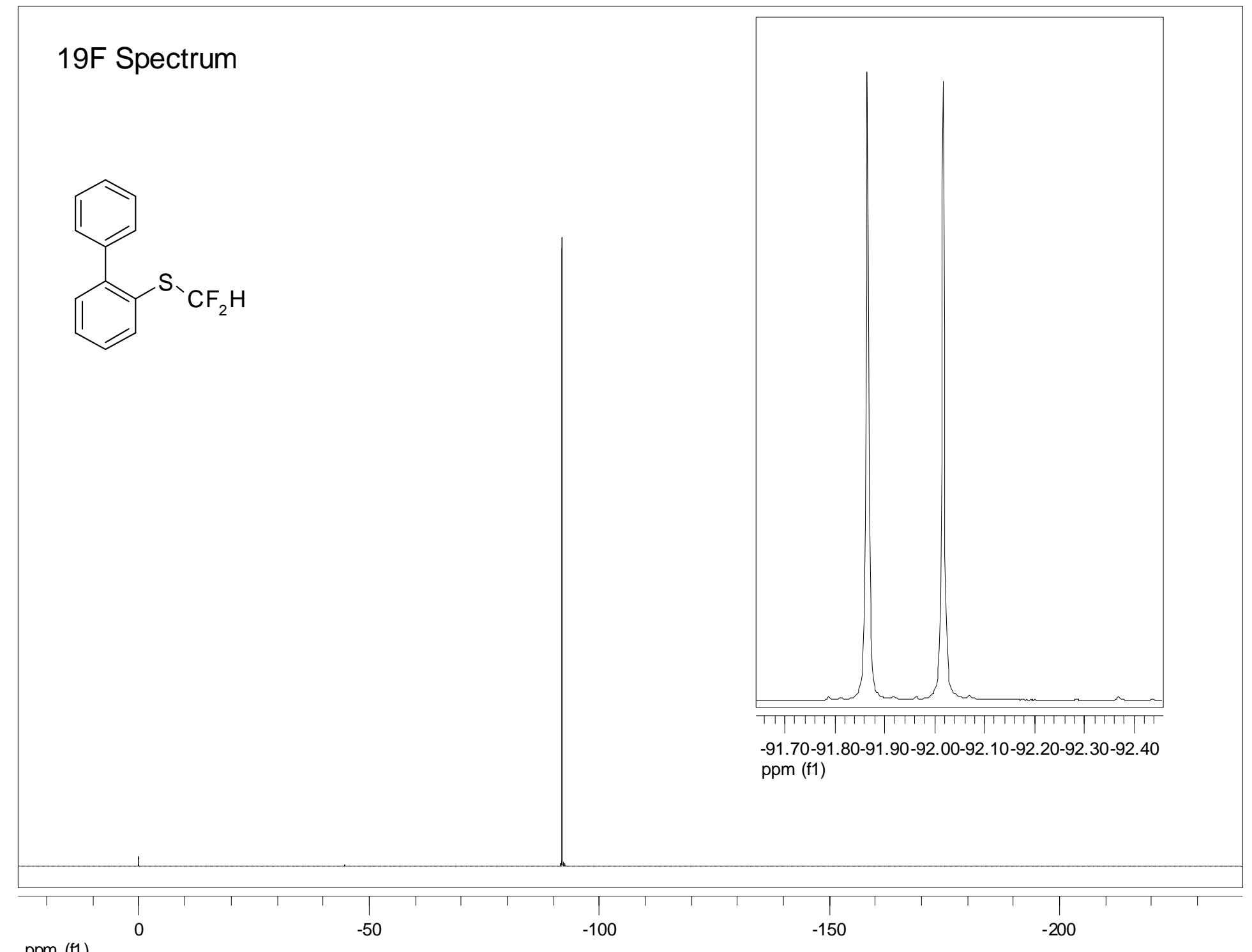

ppm (f1) 


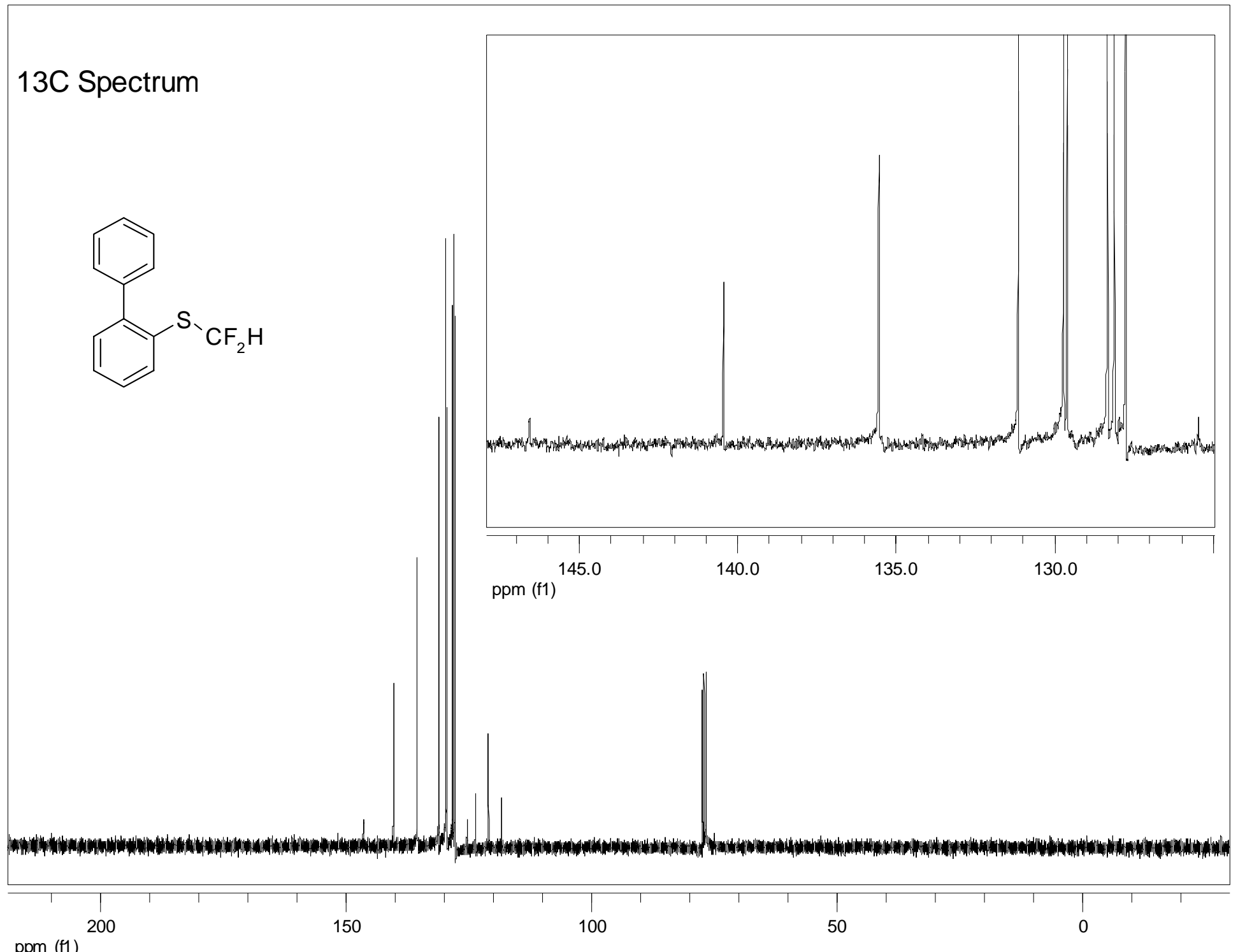

ppm (f1) 


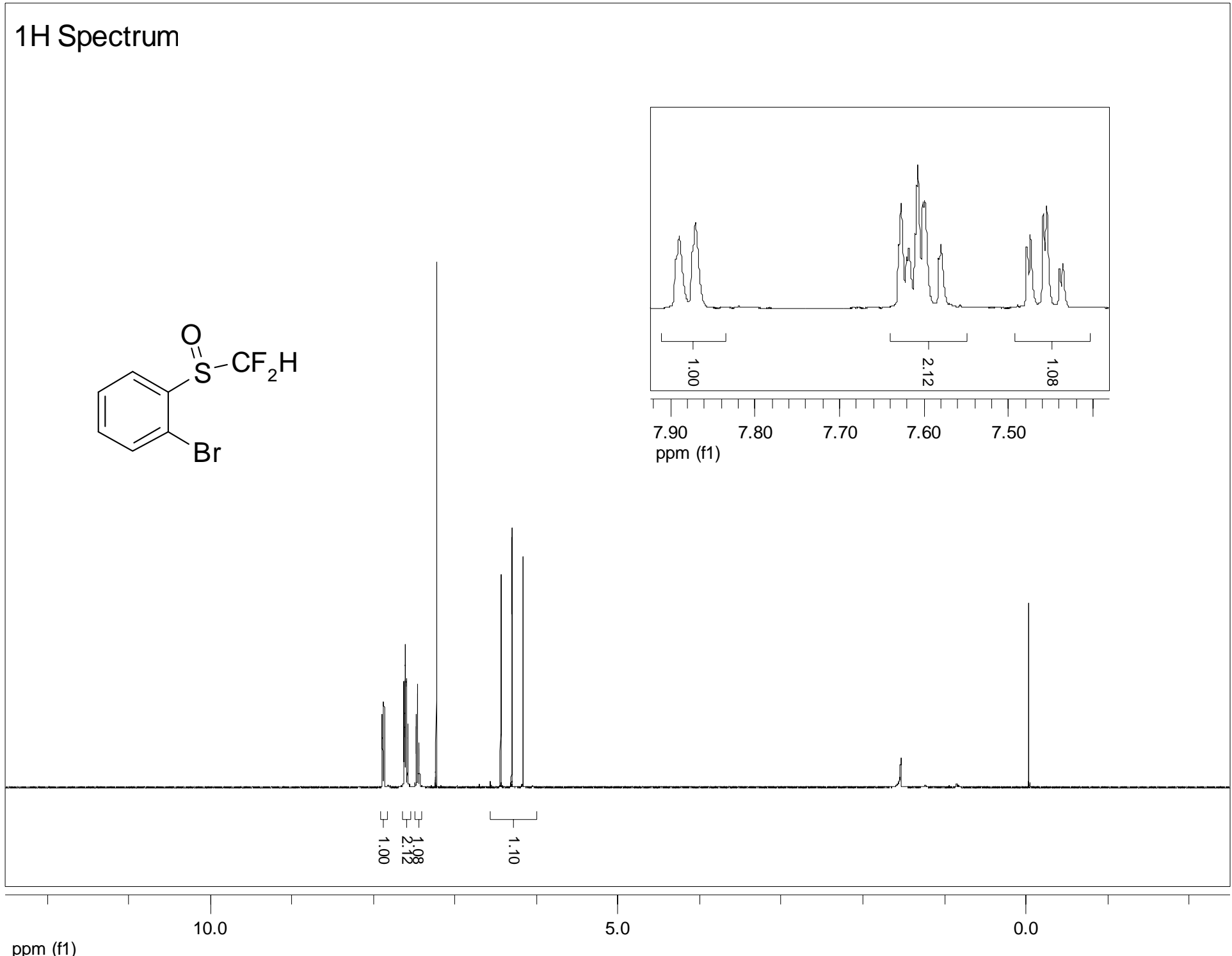

- S24 - 


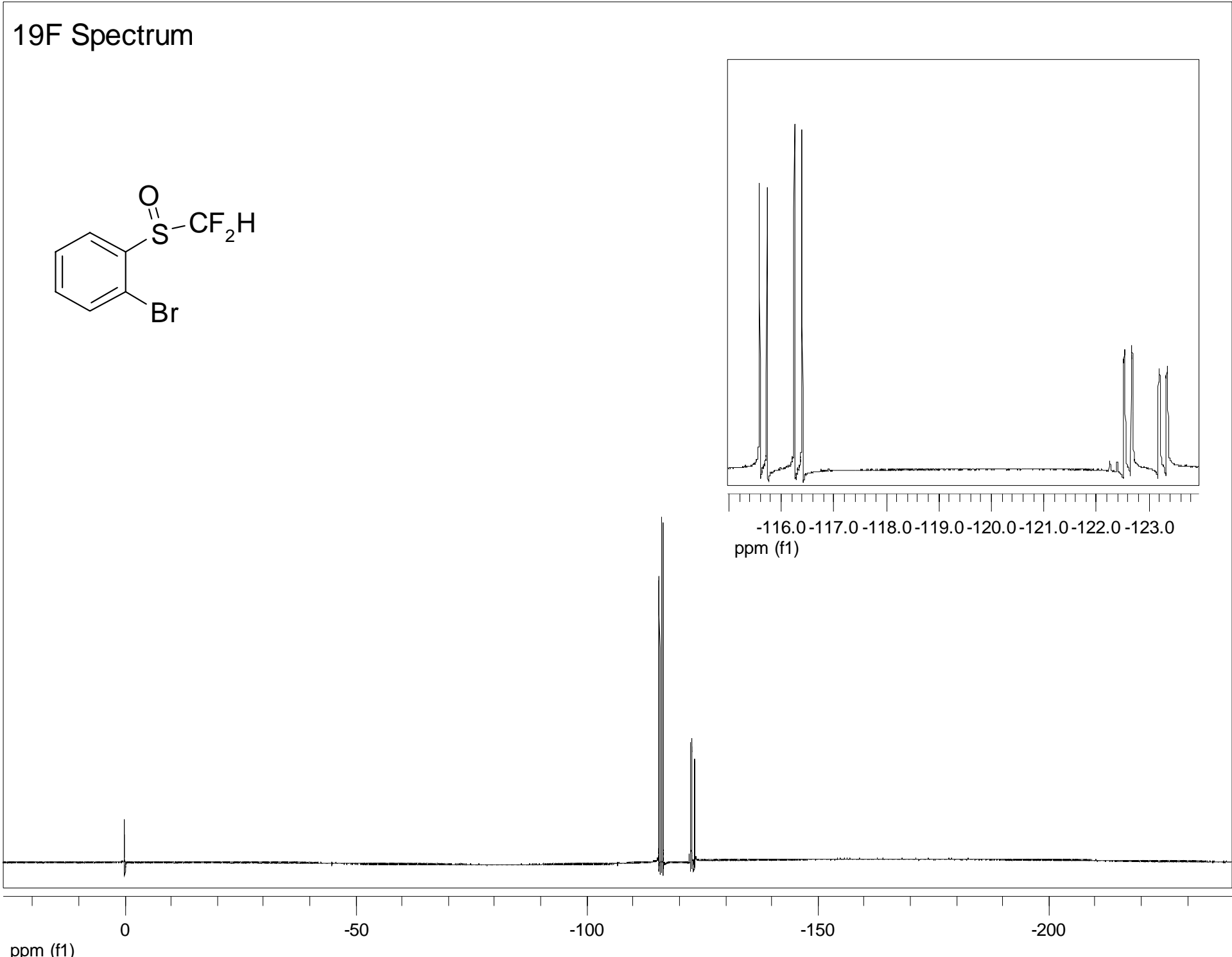

- S25 - 


\section{C Spectrum}

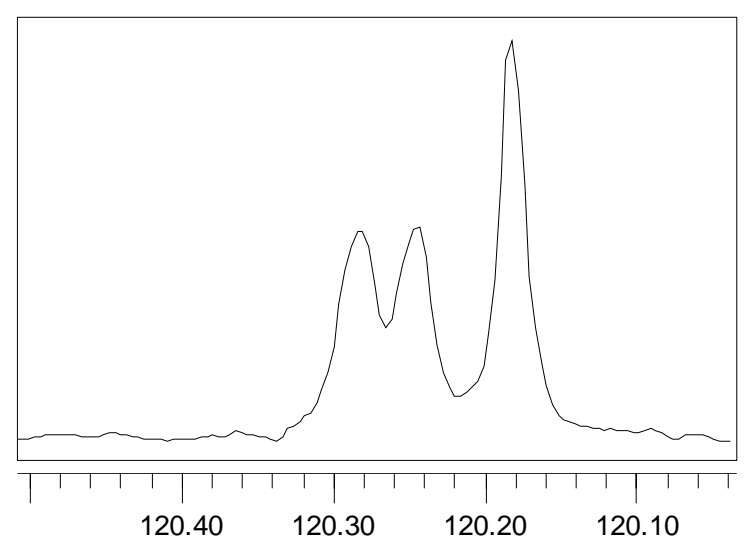

ppm (f1)

120.30
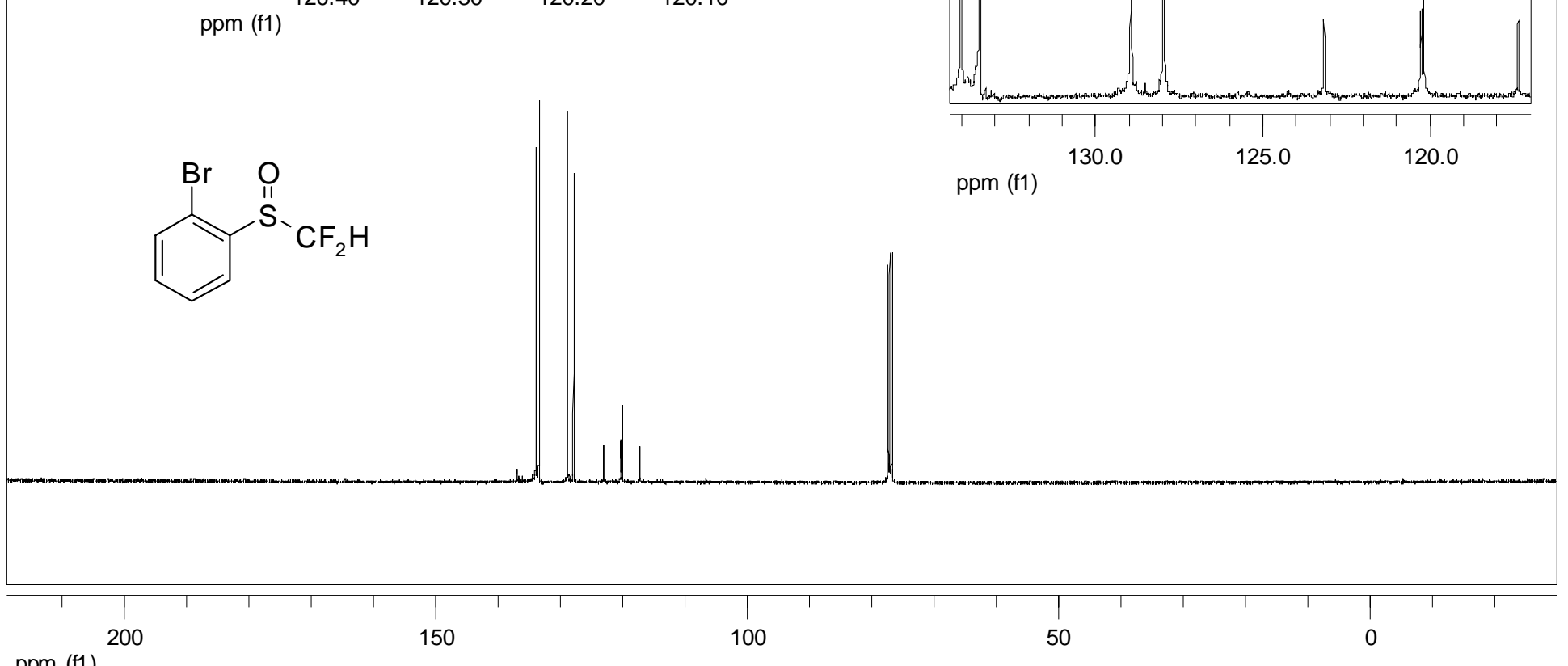

ppm (f1) 


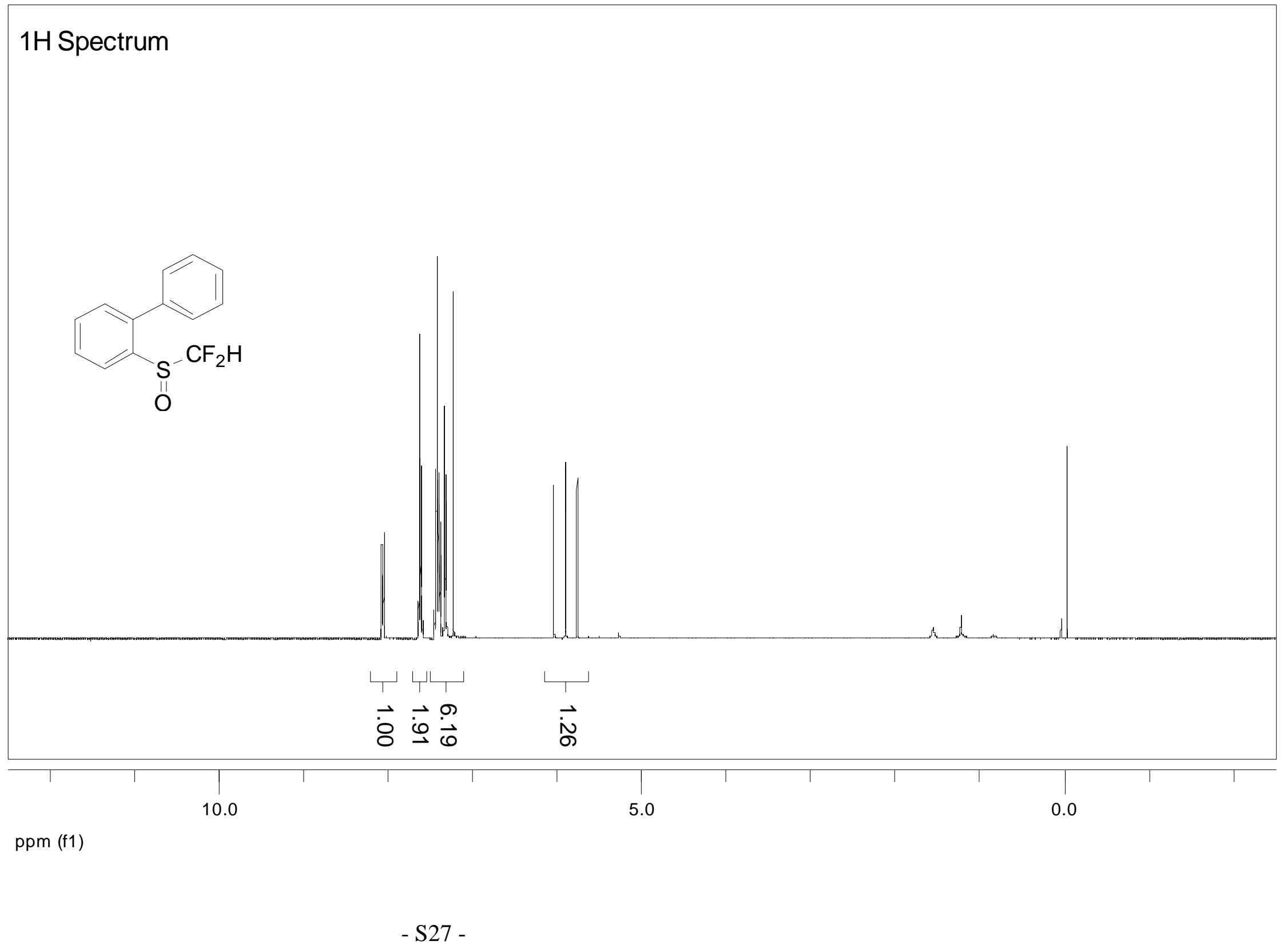




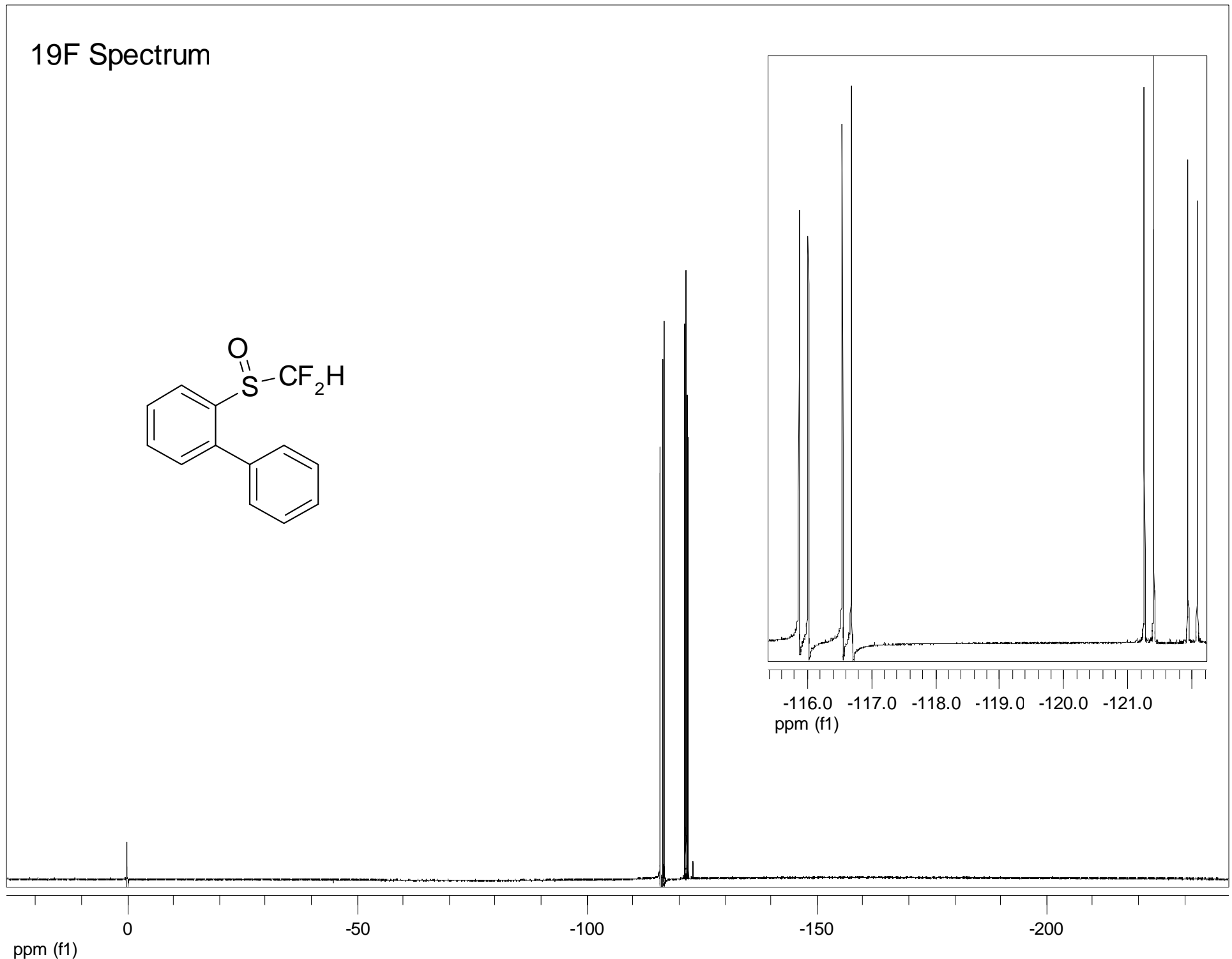

- S28 - 


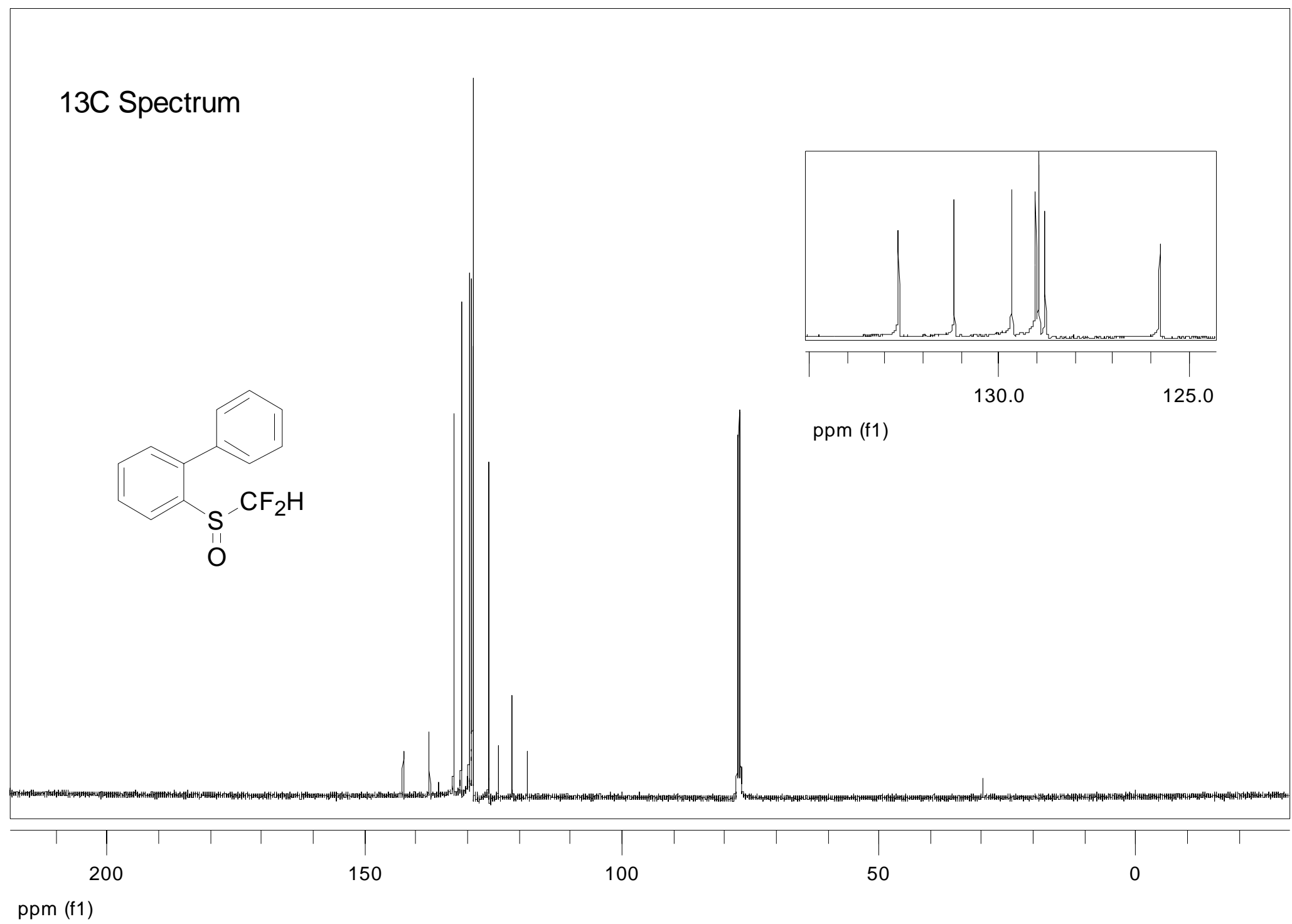

- S29 - 


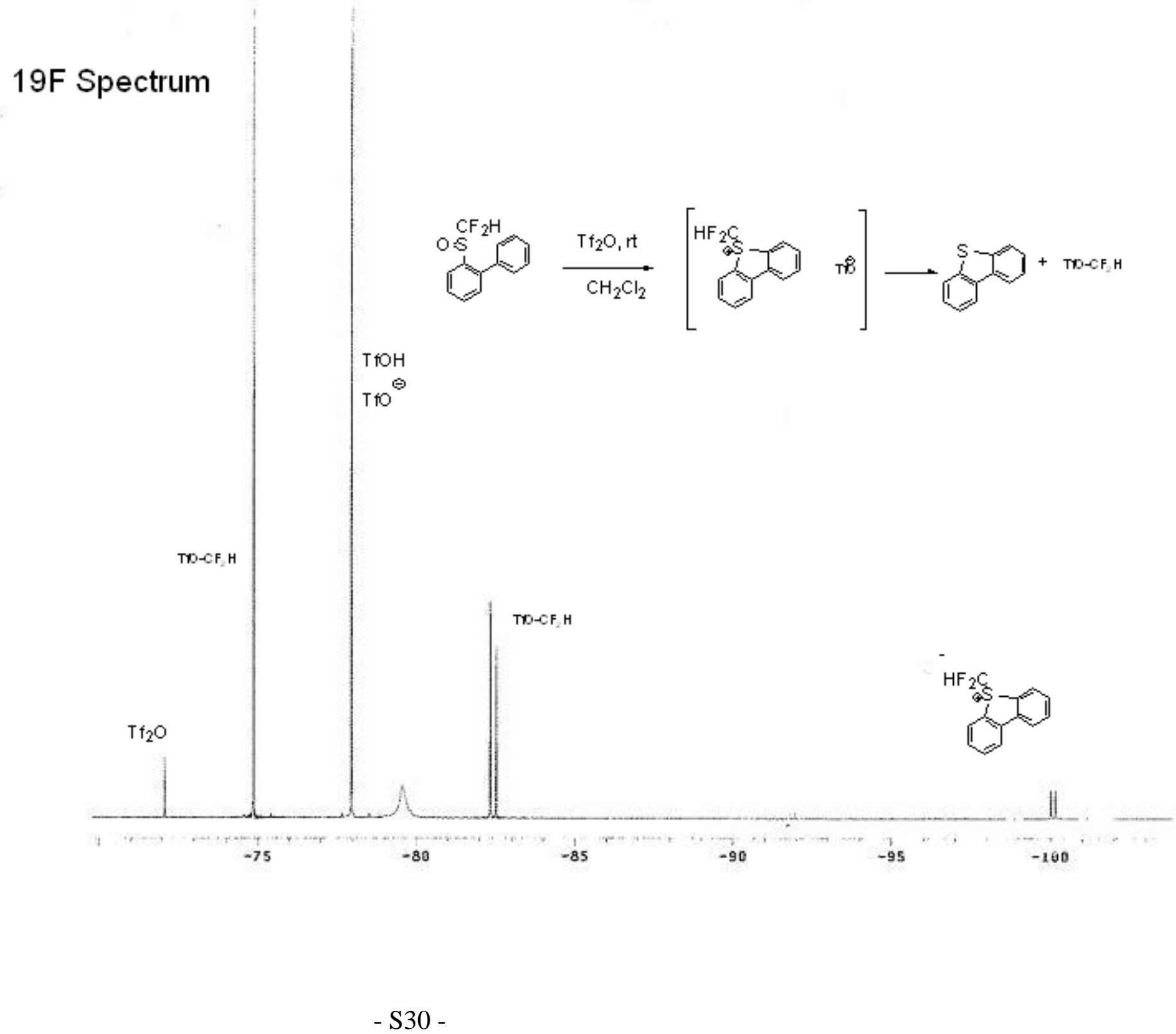




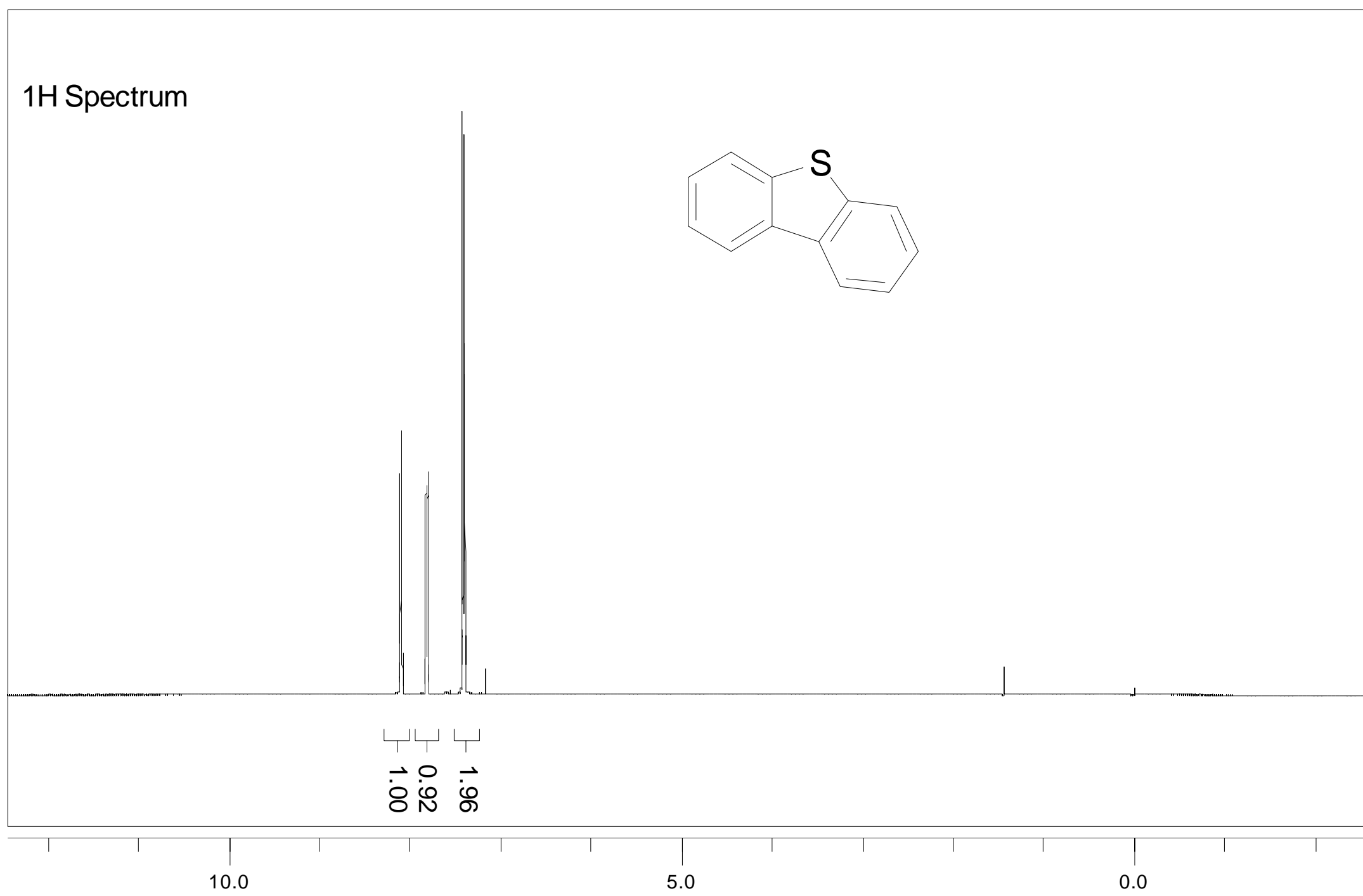

ppm (f1) 


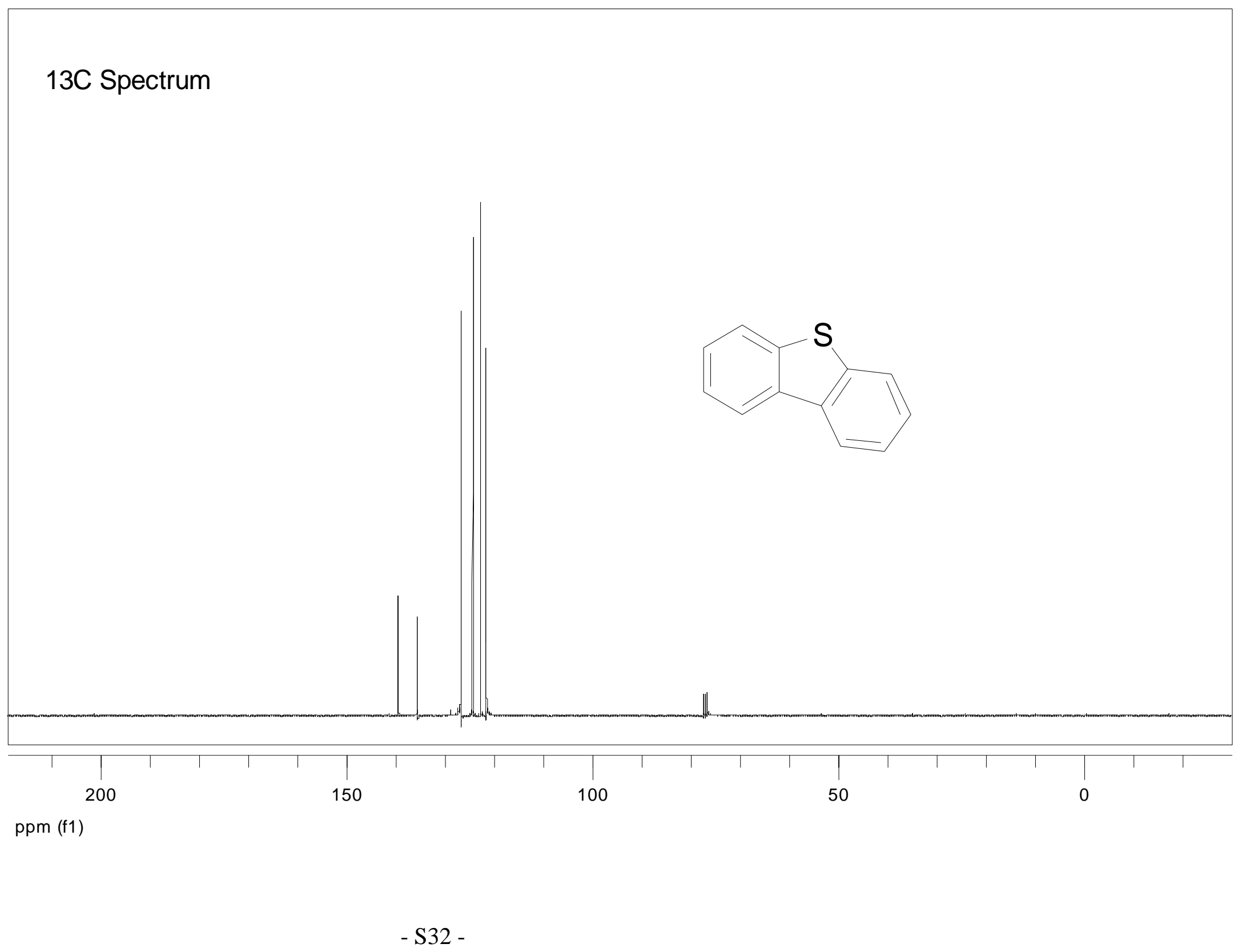




\section{F Spectrum in CD3CN}

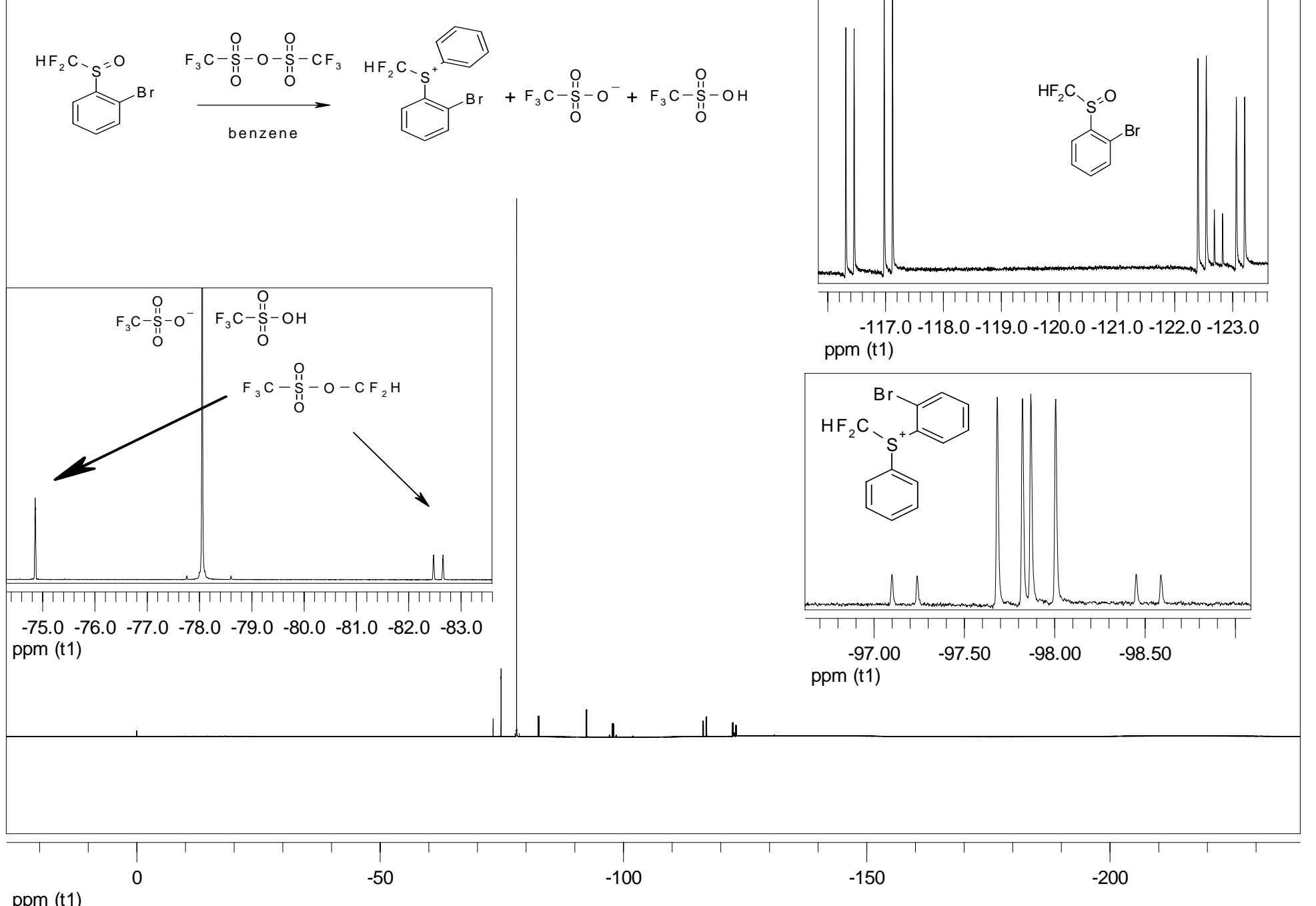

ppm (t1) 


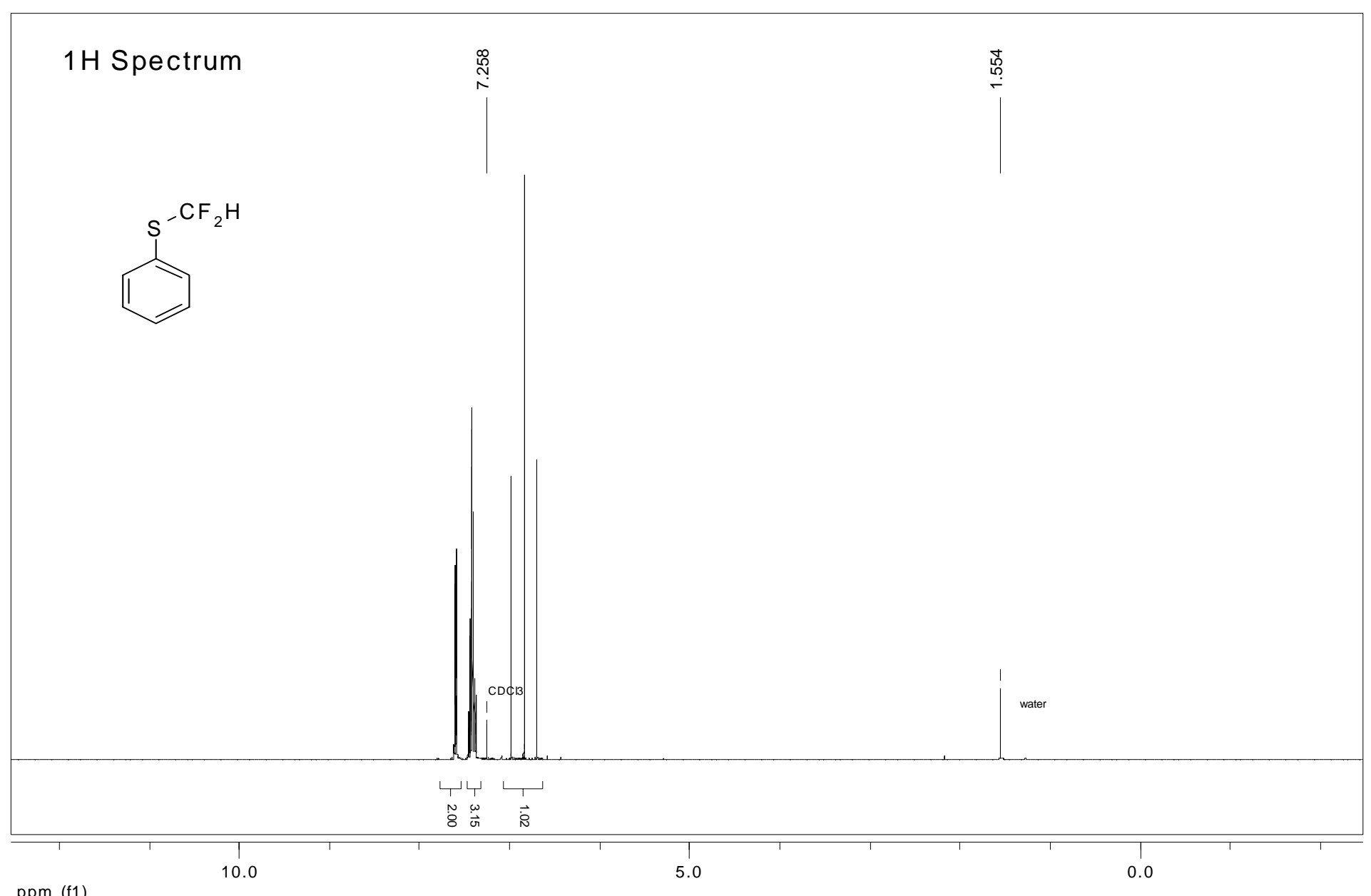

- S34 - 


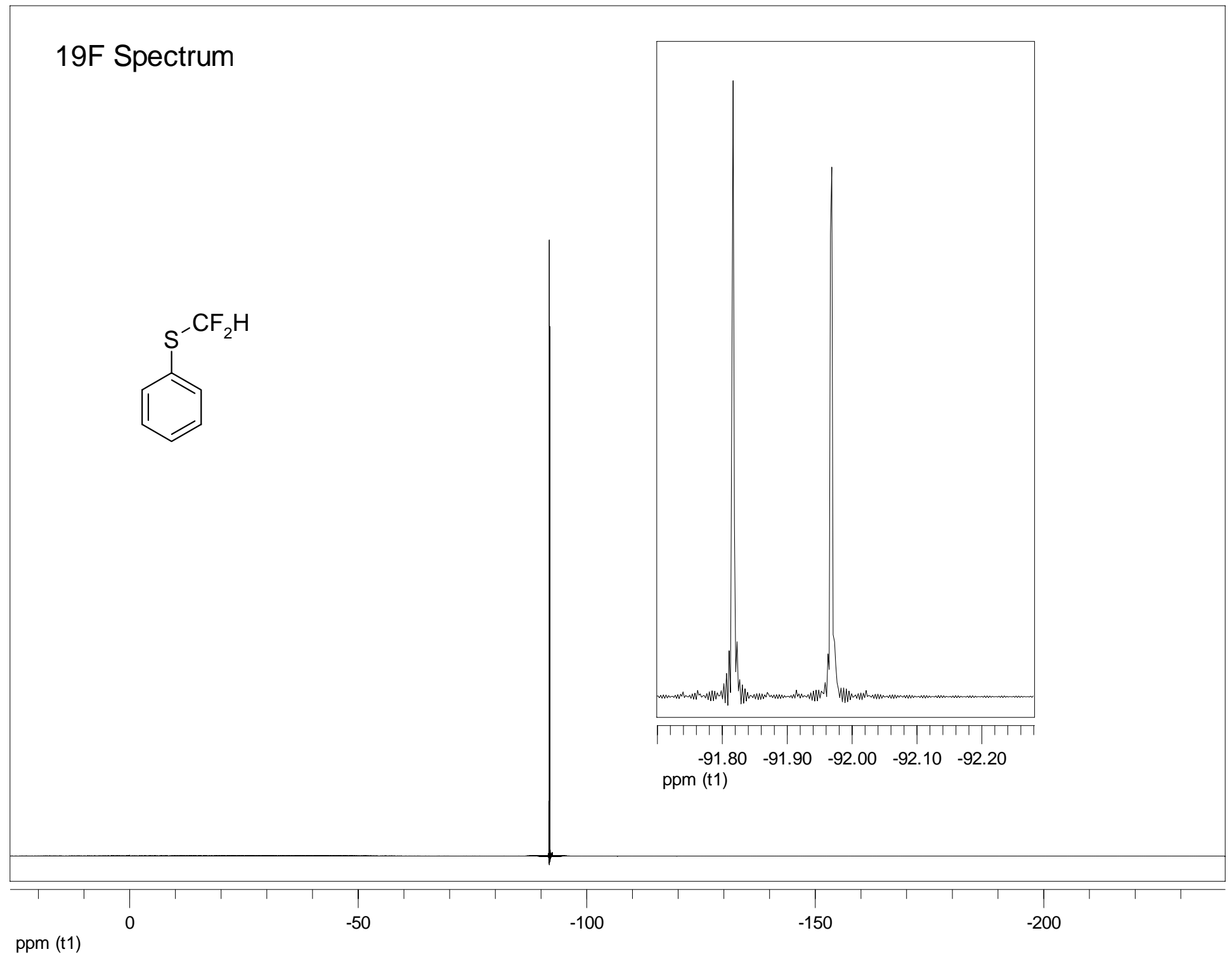

- S35 - 


\section{C Spectrum}
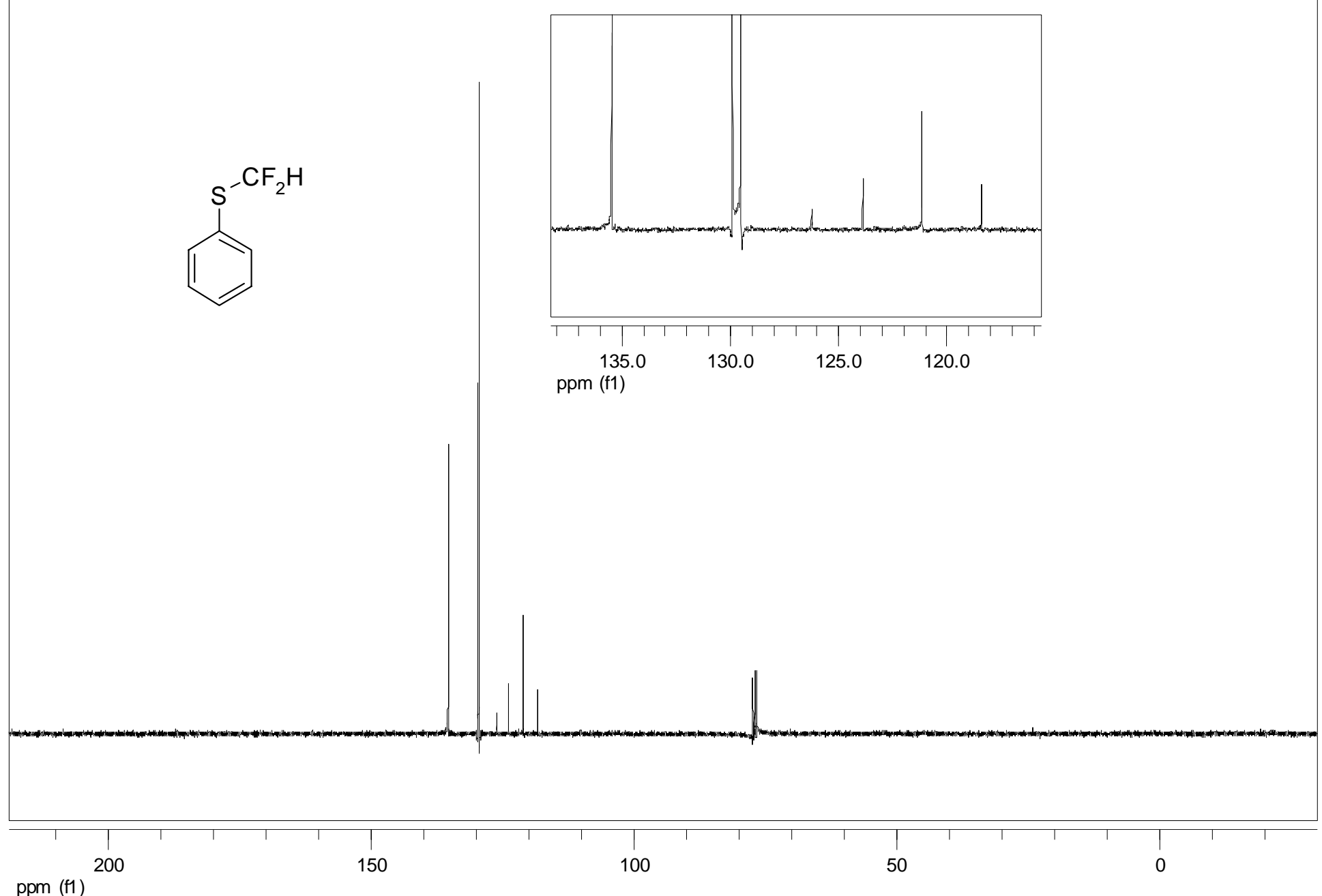

ppm (11)

- S36 - 


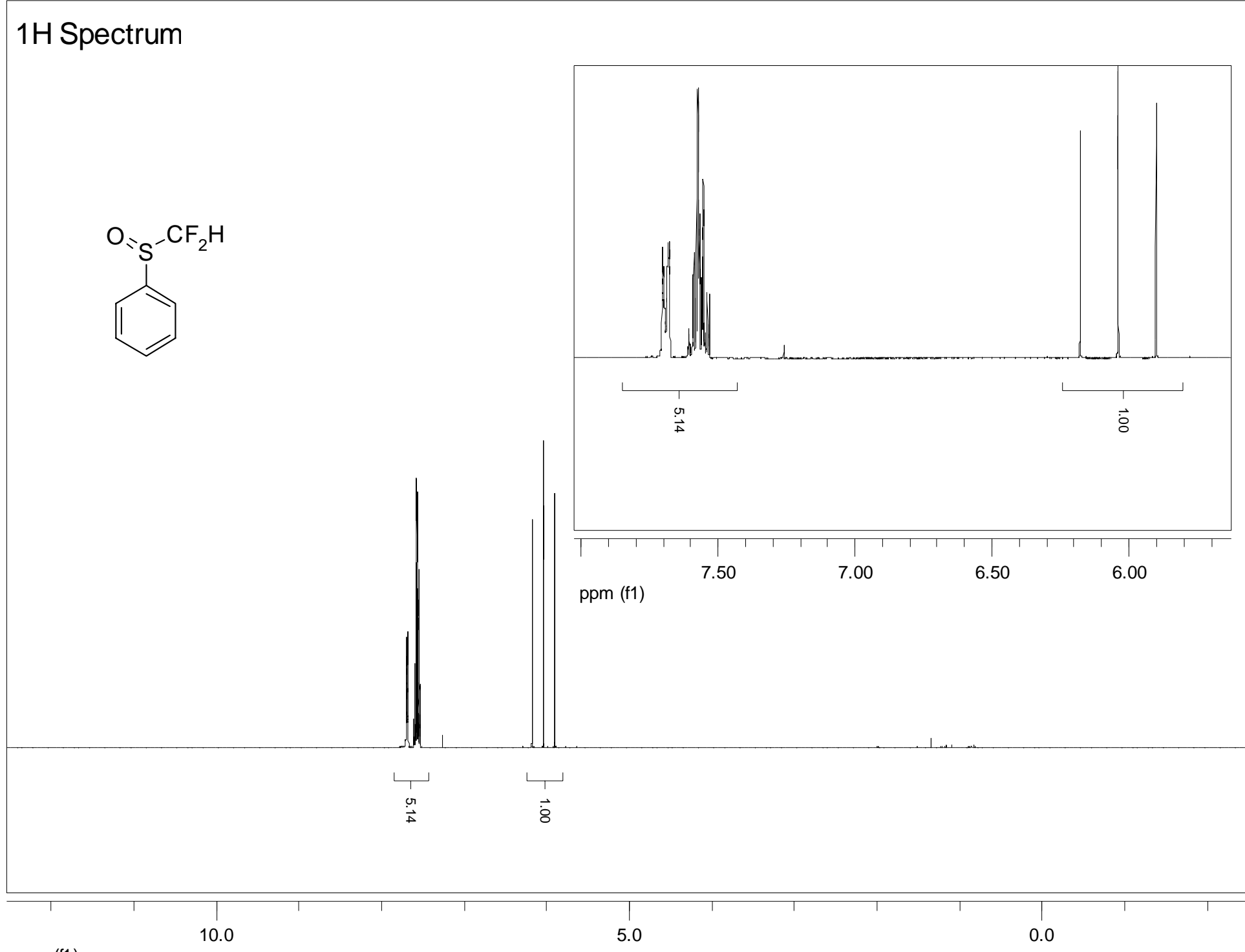

ppm (f1)

- S37 - 


\section{F Spectrum}

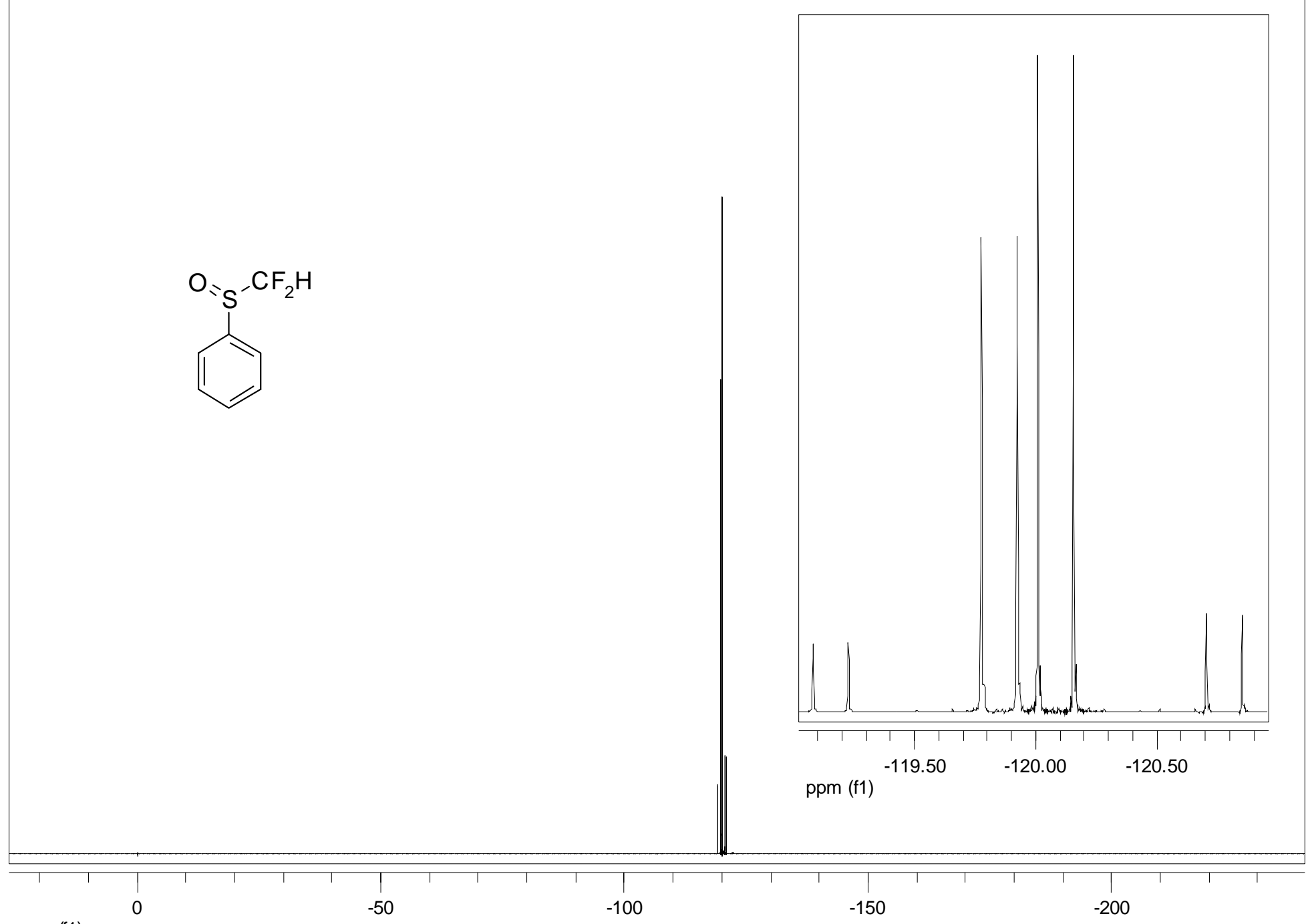

ppm (f1)

- S38 - 
13C Spectrum

$\mathrm{O}$ s $-\mathrm{CF}_{2} \mathrm{H}$

1

ppm (f1)

- S39 - 


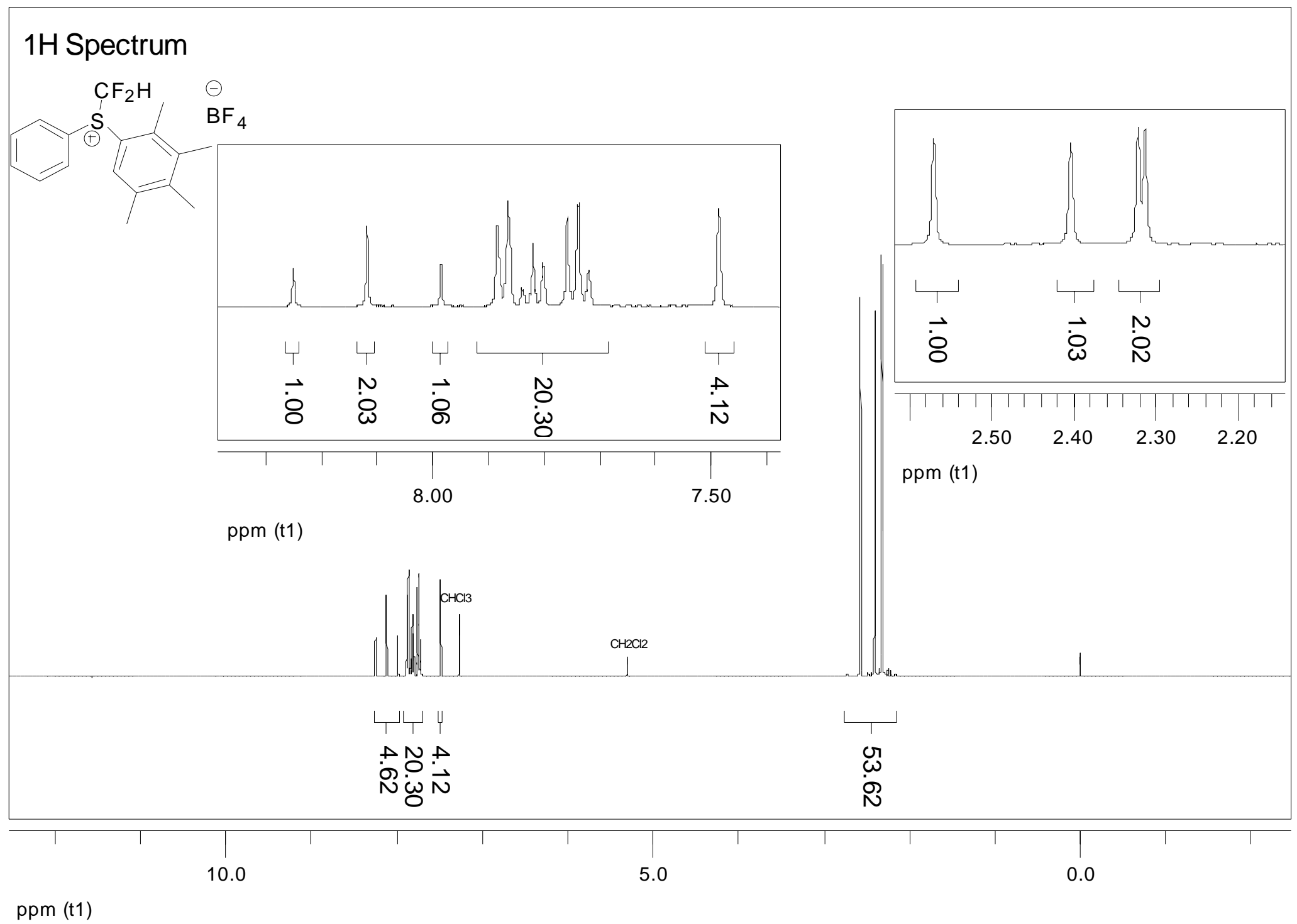


19F Spectrum
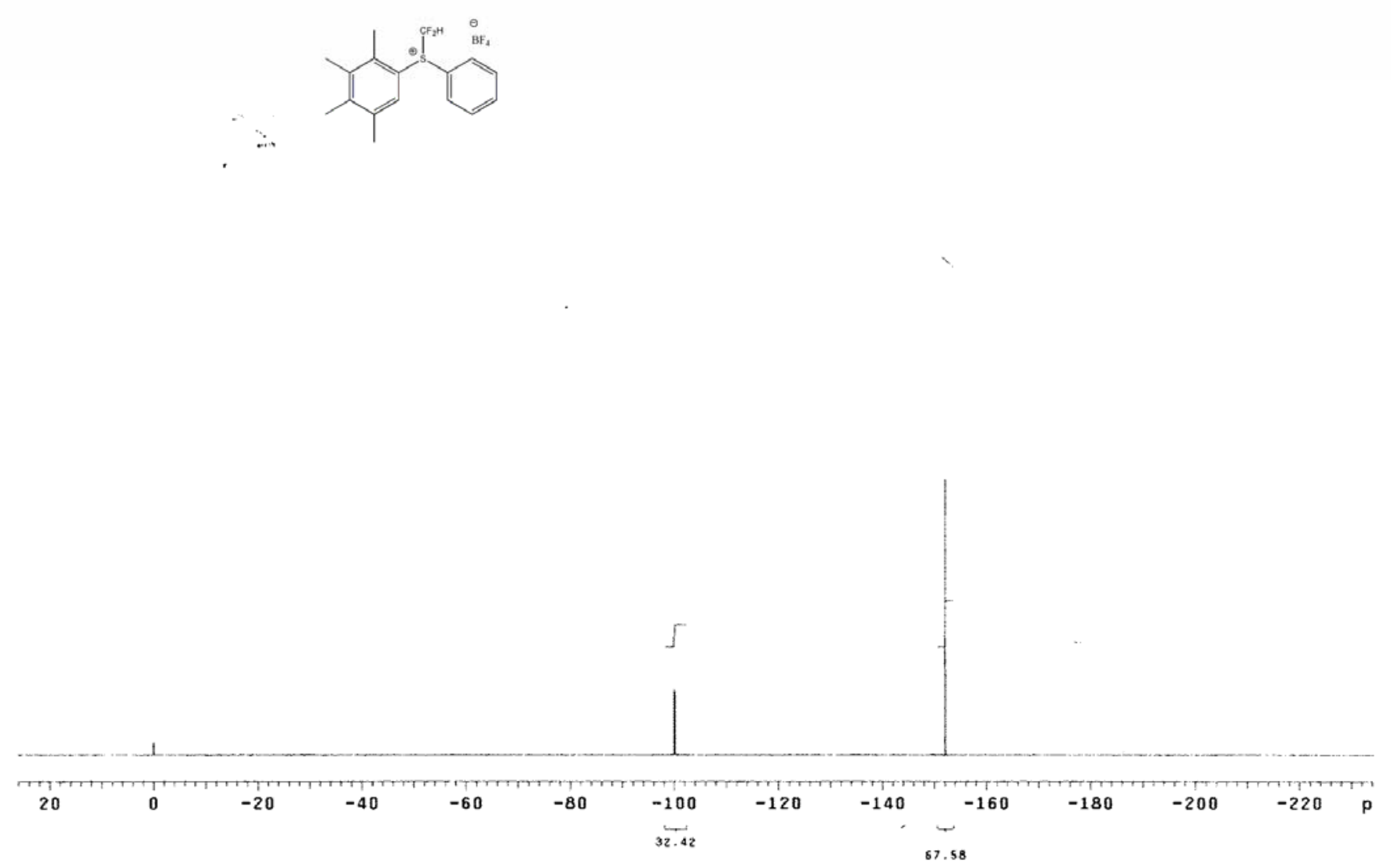

- S41 - 
(......

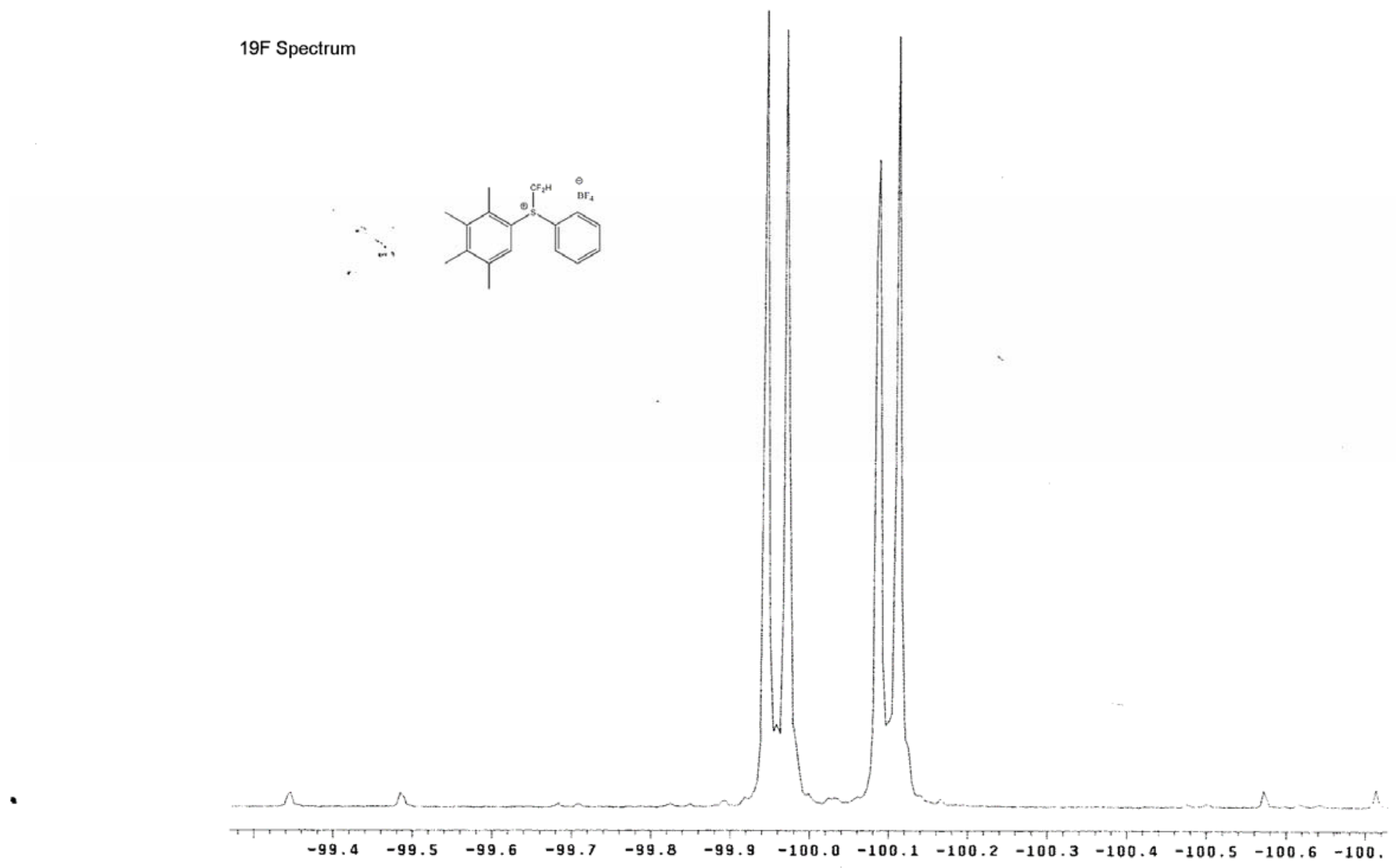

- S42 - 
19F Spectrum

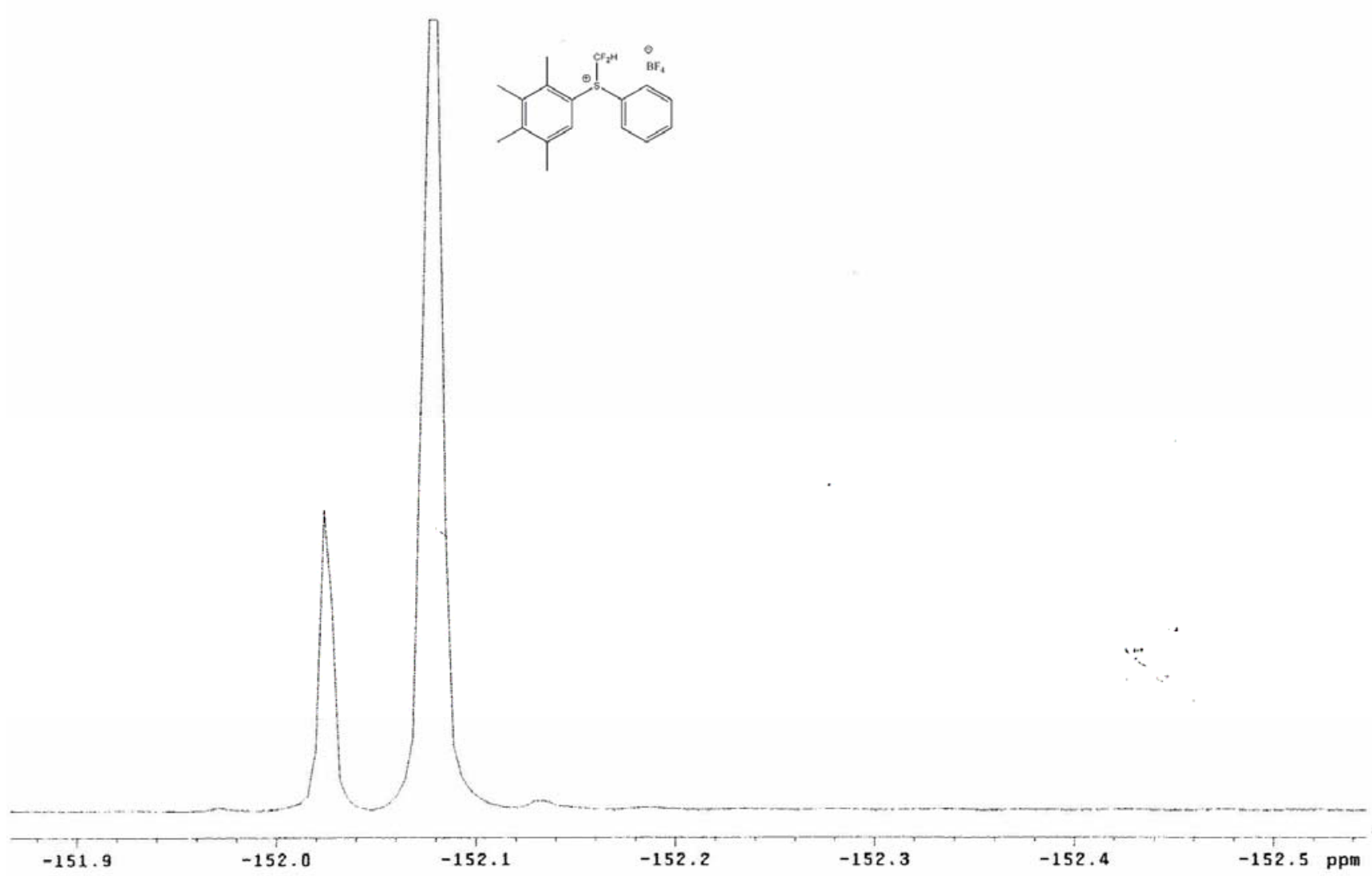




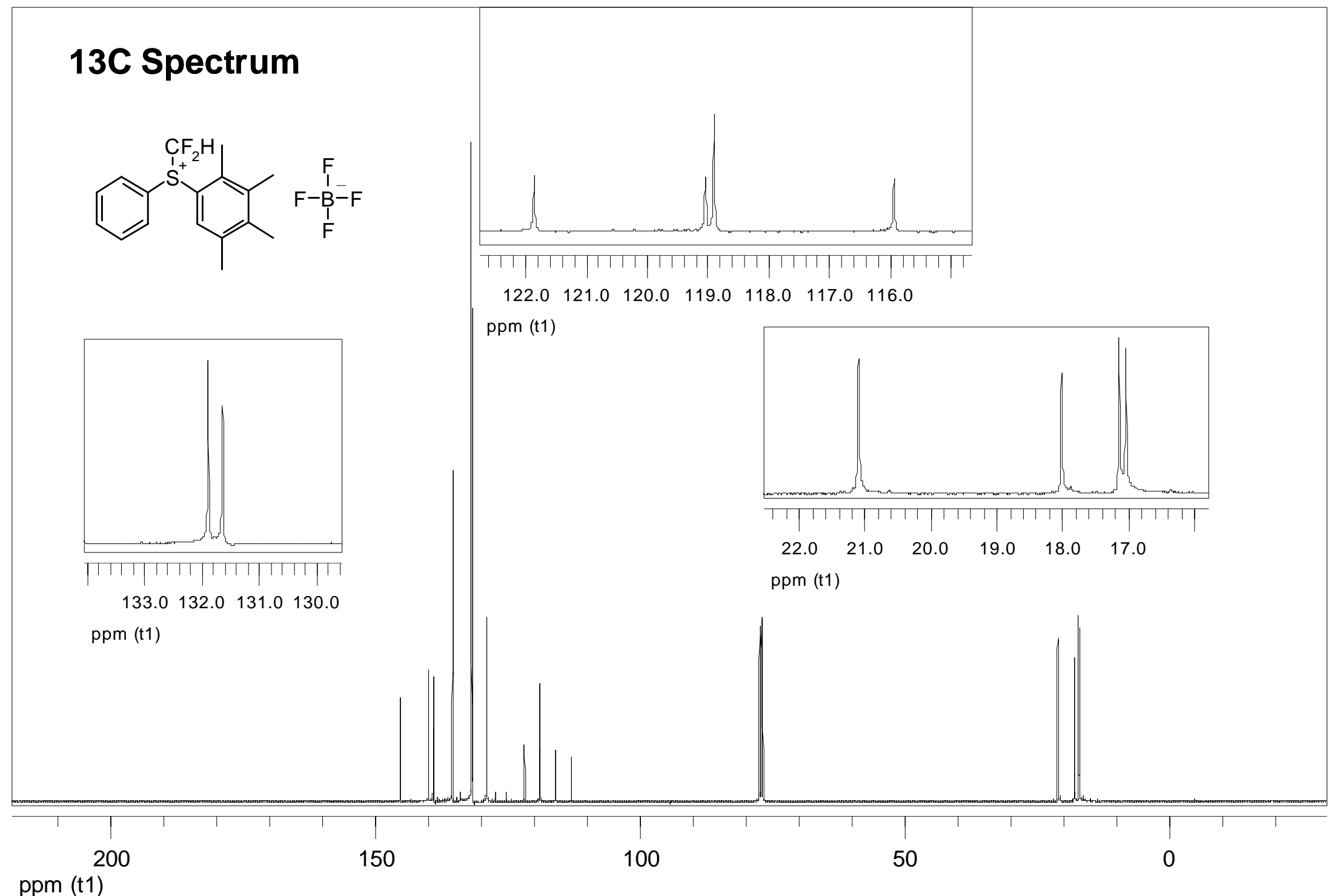

- S44 - 


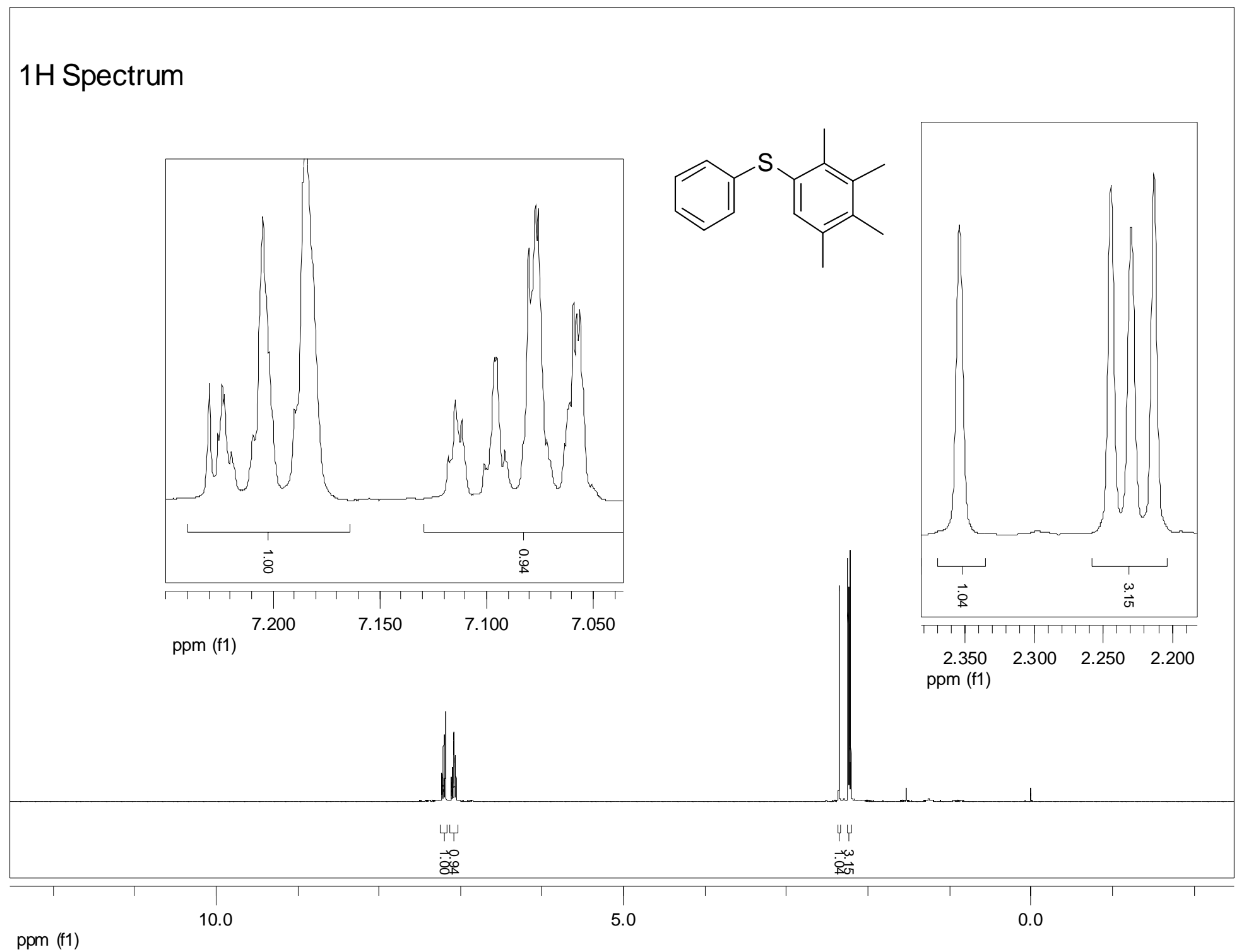

- S45 - 


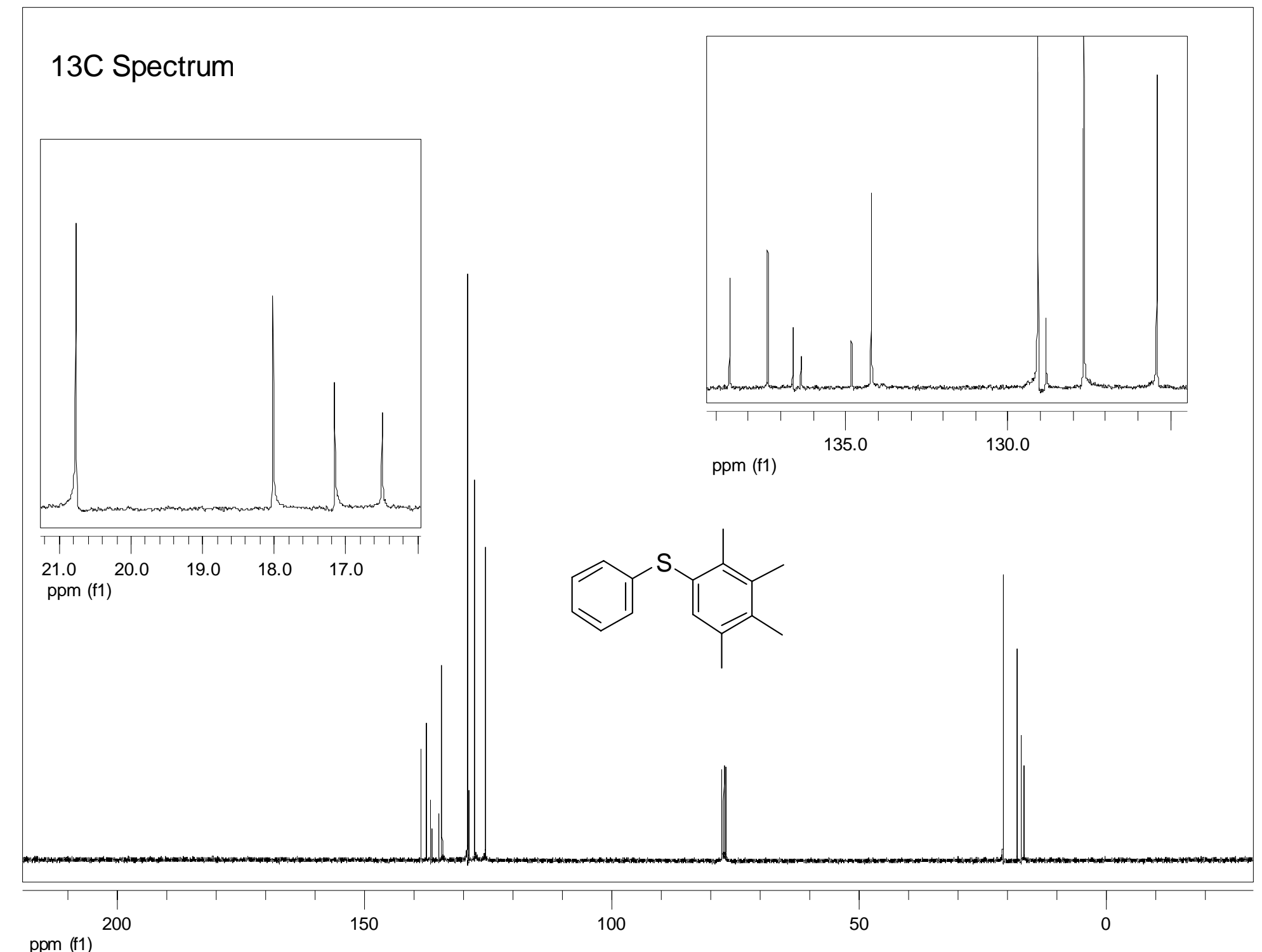

ppm (f1)

- S46 - 


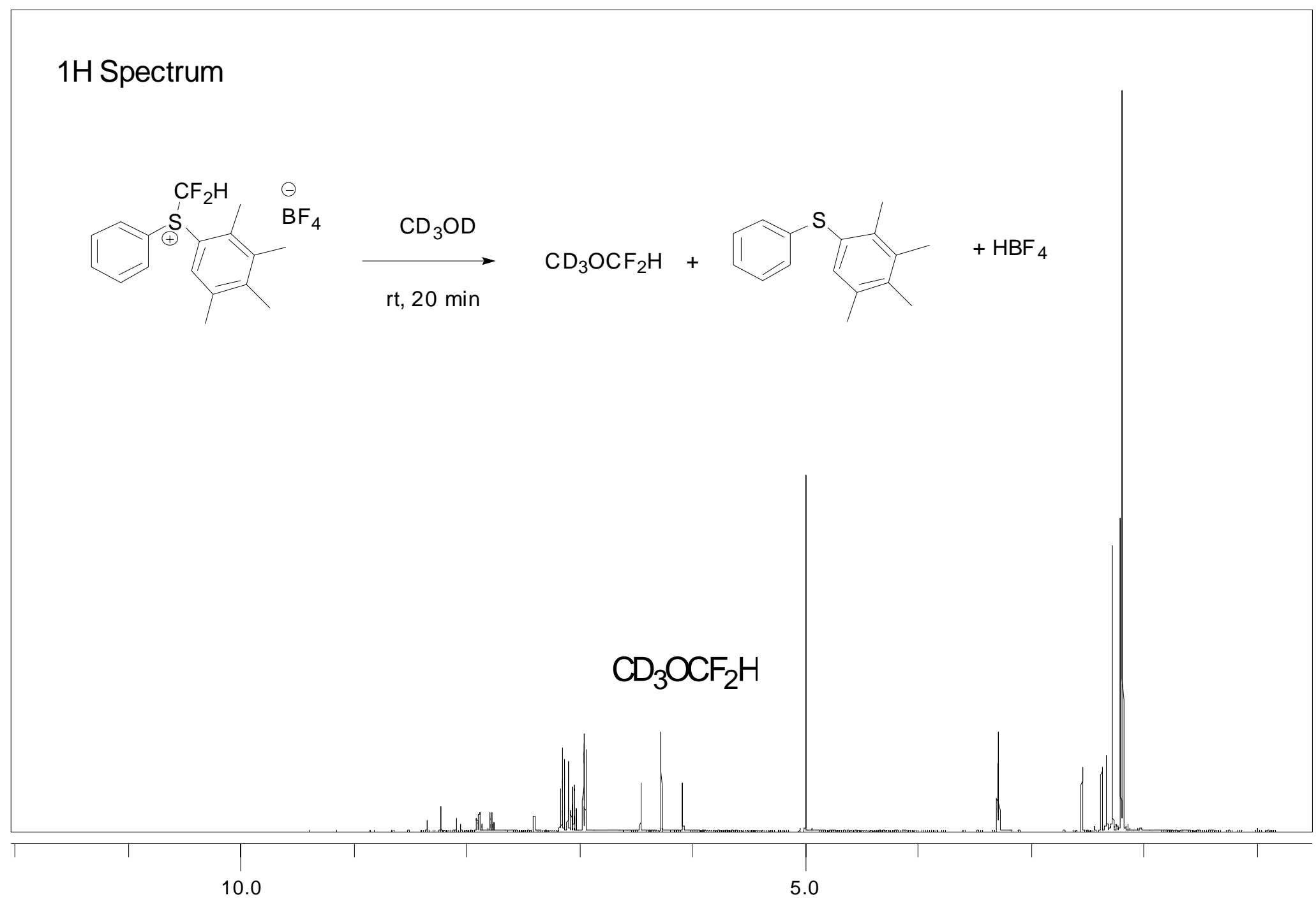

ppm (f1)

- S47 - 


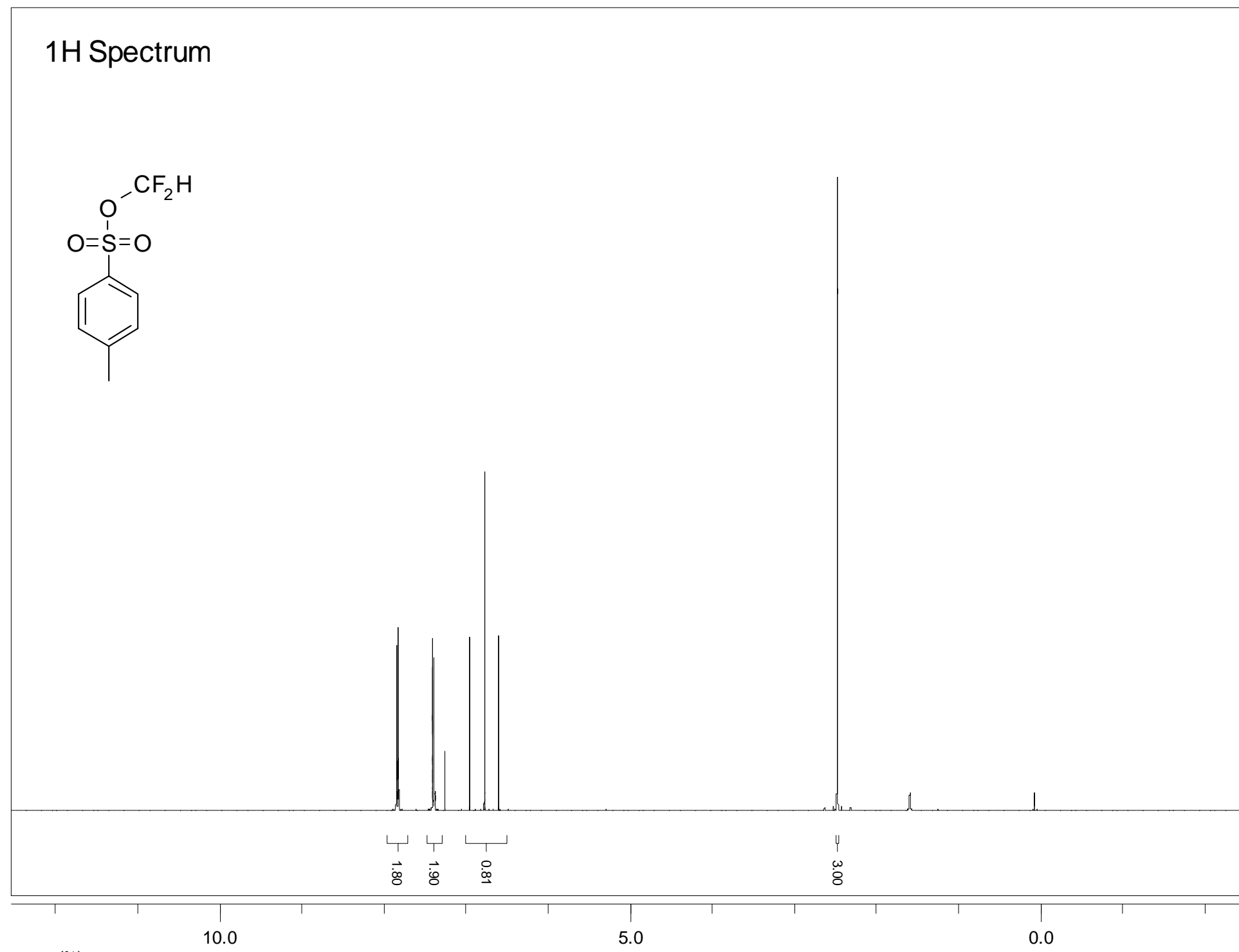

ppm (f1)

- S48 - 


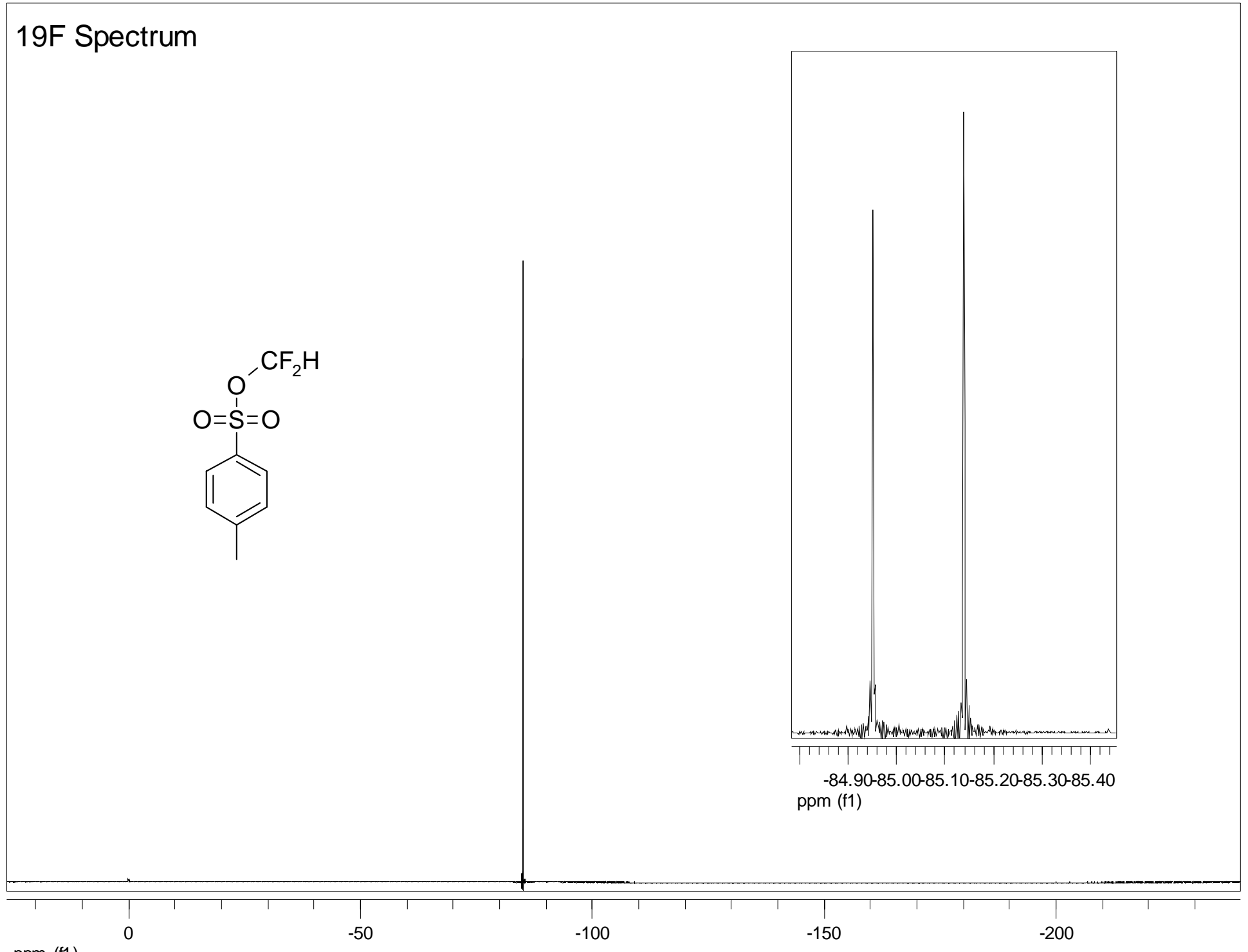

ppm (f1) 


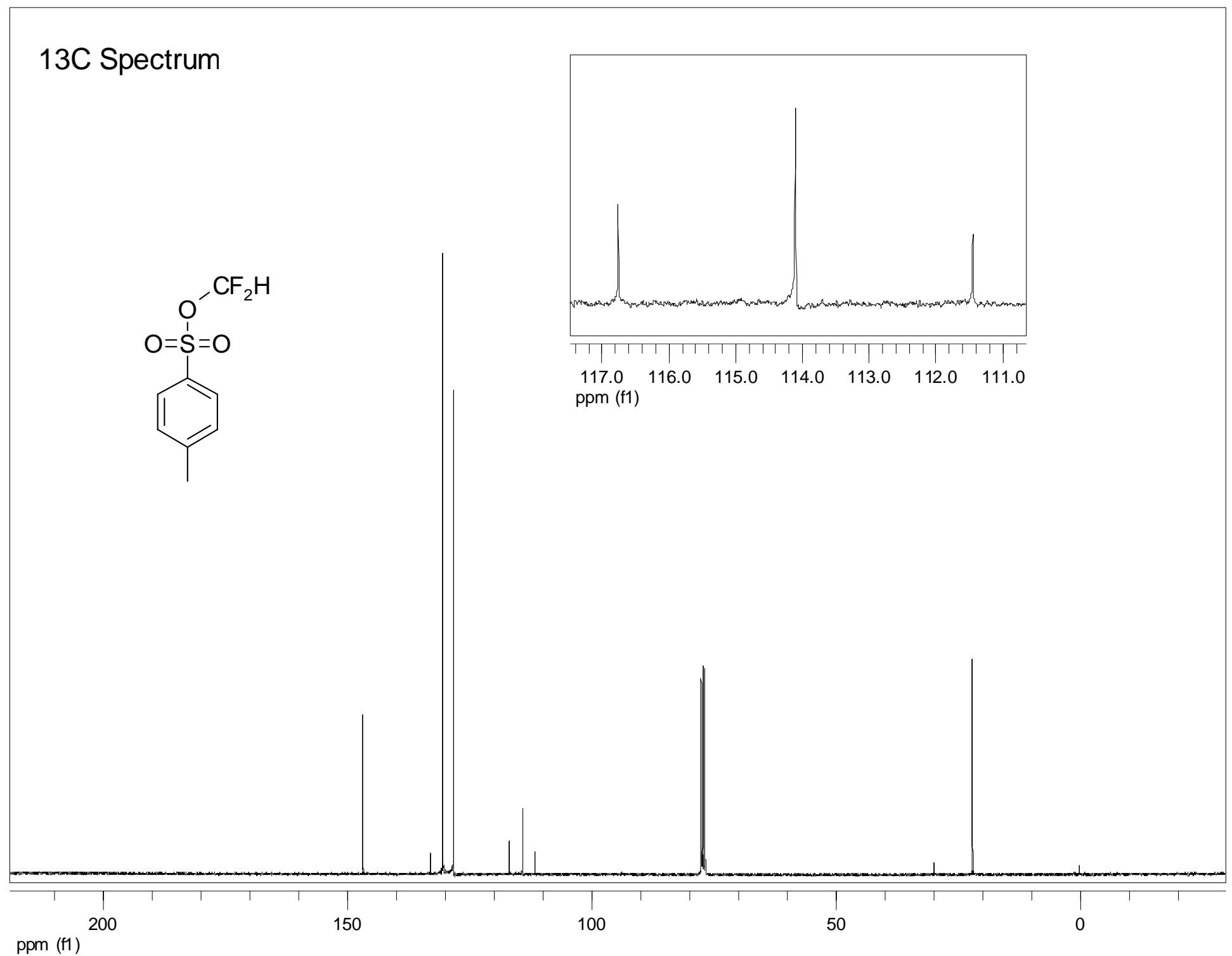

- S50 - 


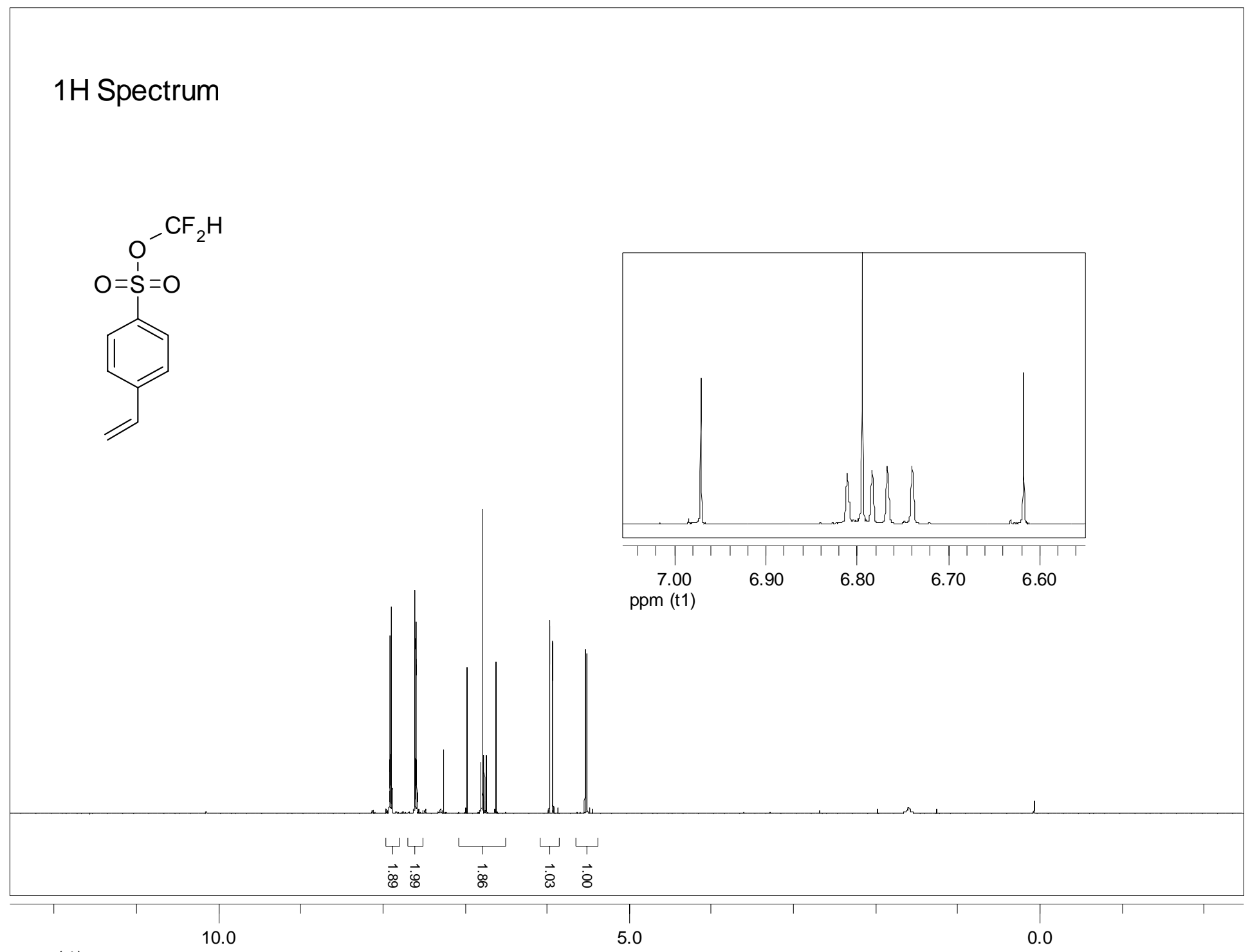

ppm (t1)

- S51 - 


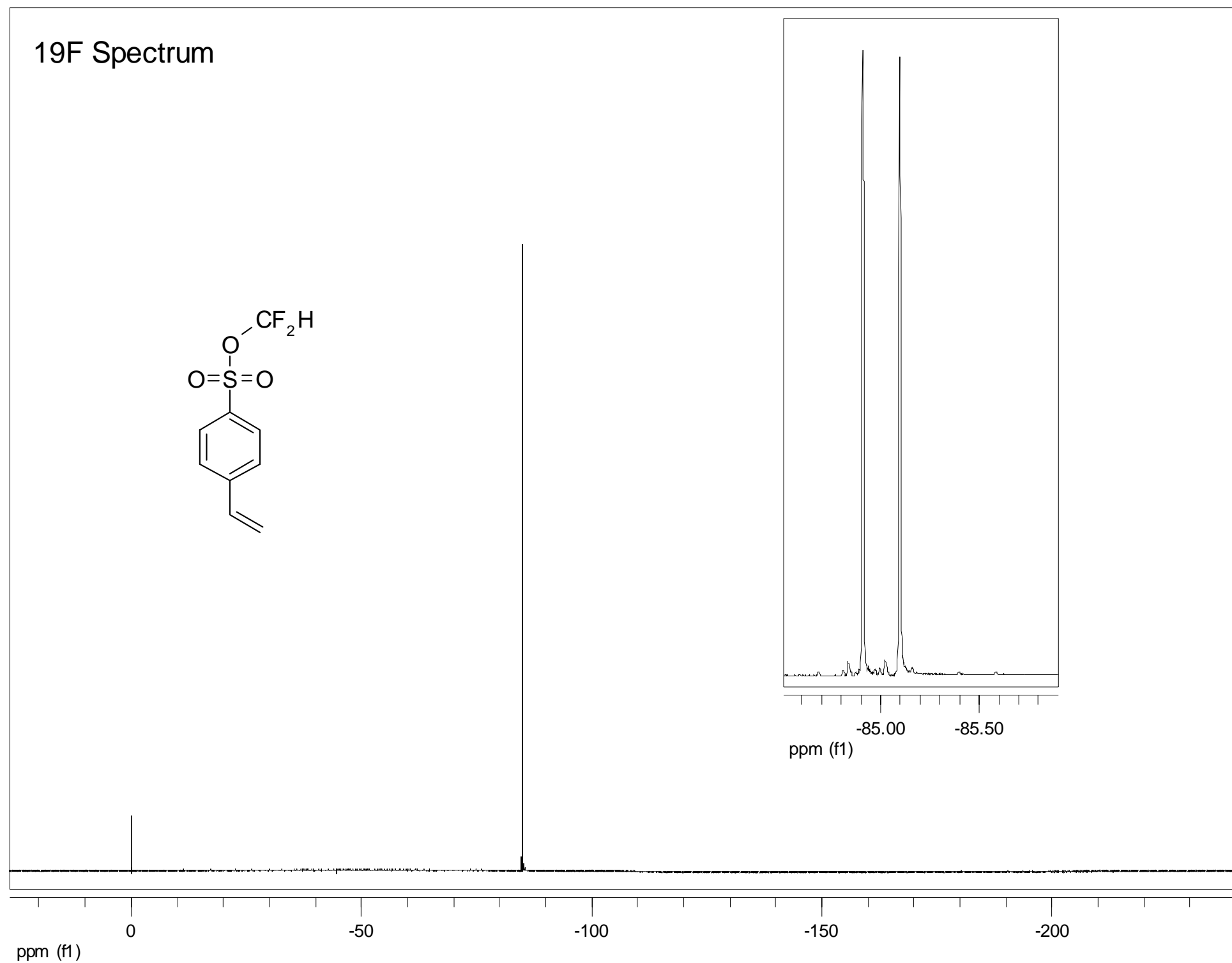

- S52 - 


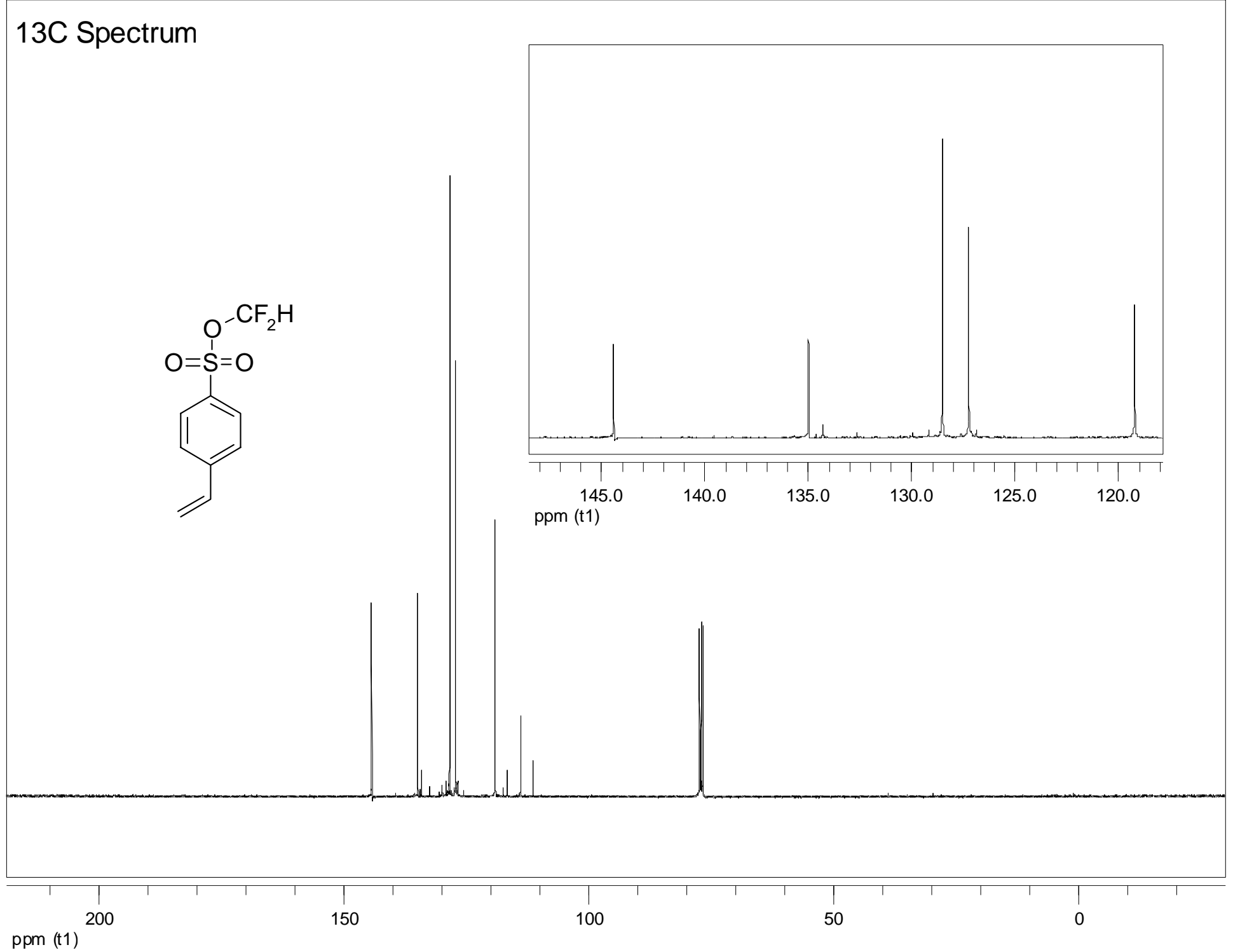

- S53 - 


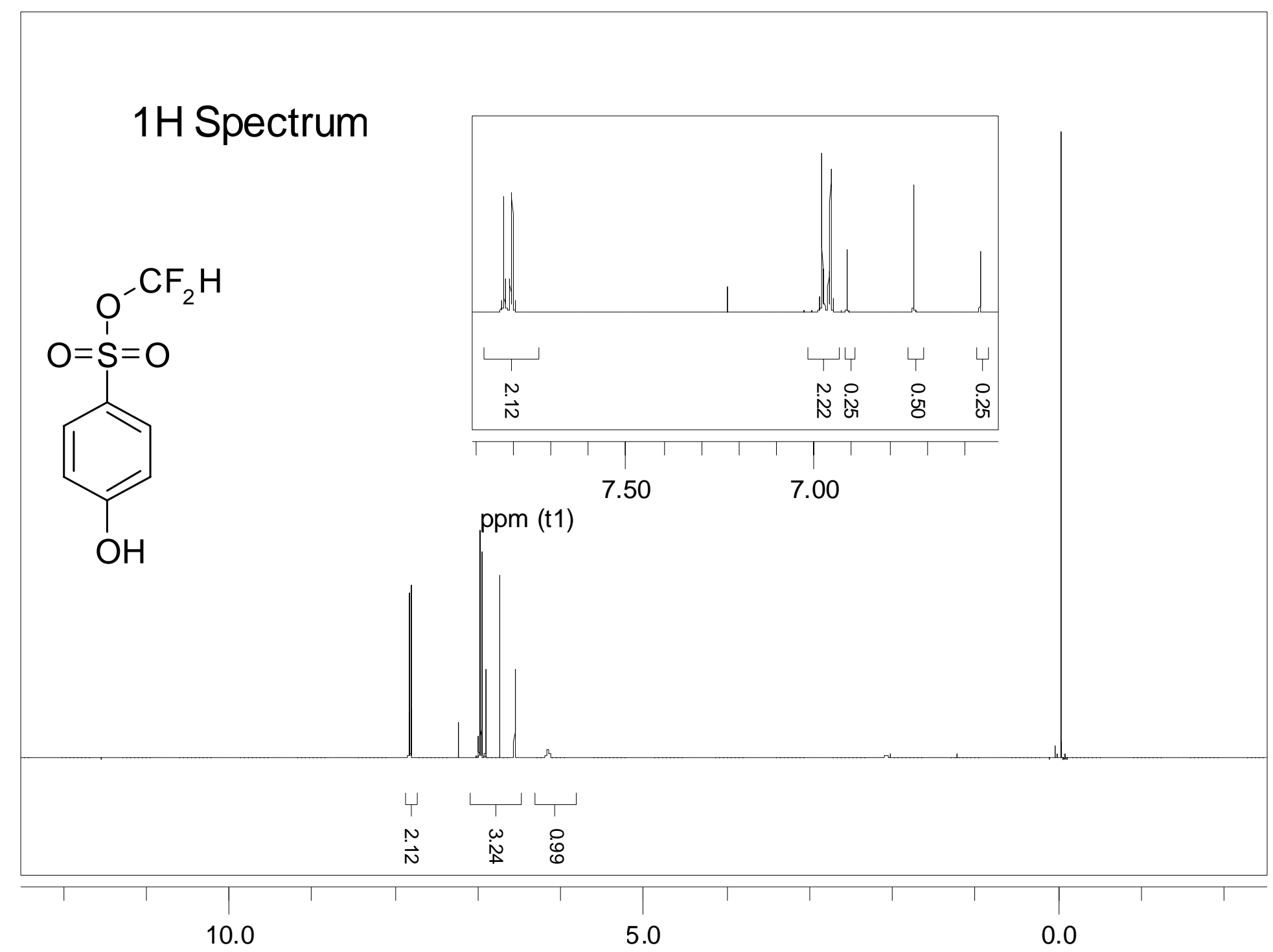

ppm (t1)

- S54 - 


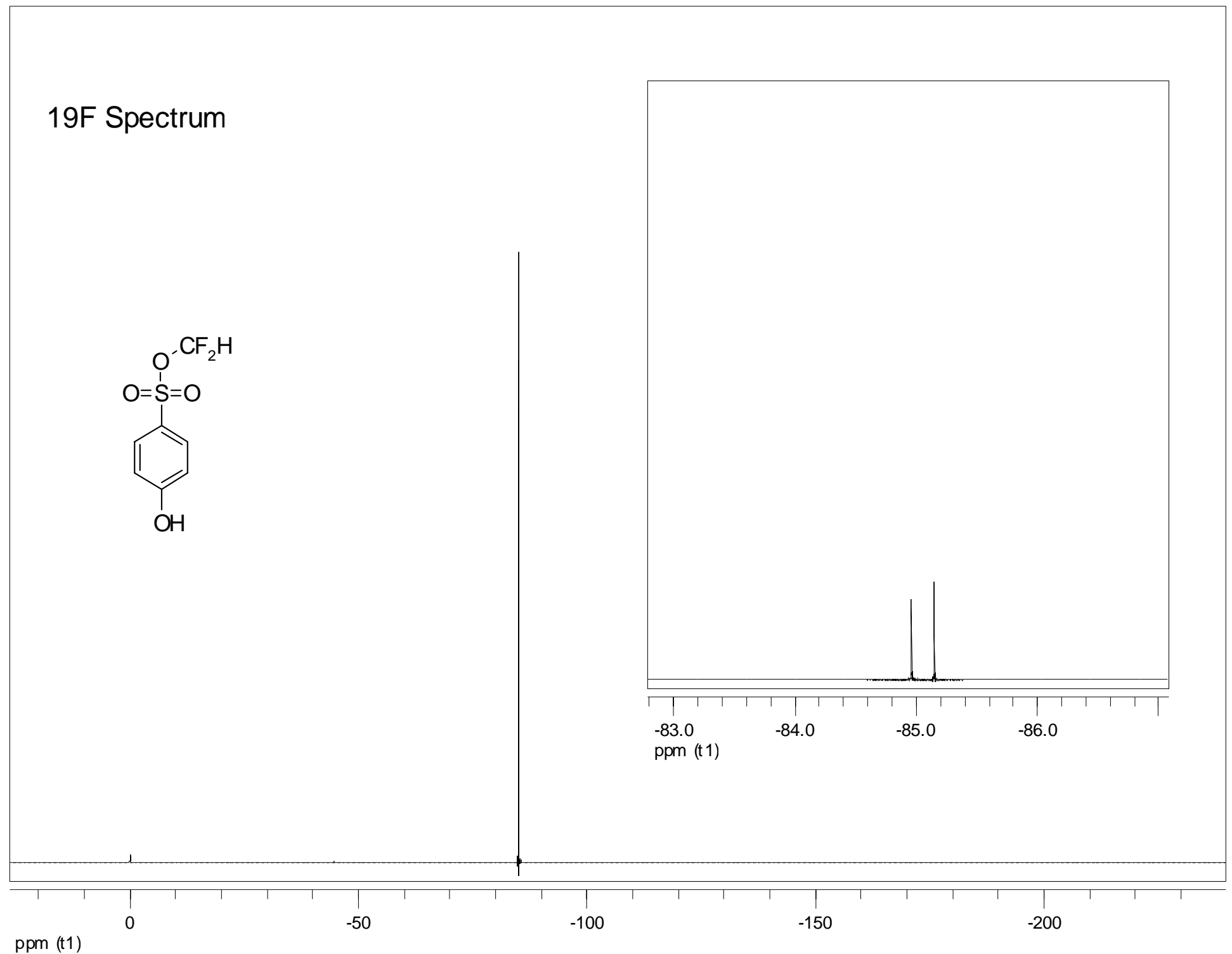

- S55 - 


\section{C Spectrum}

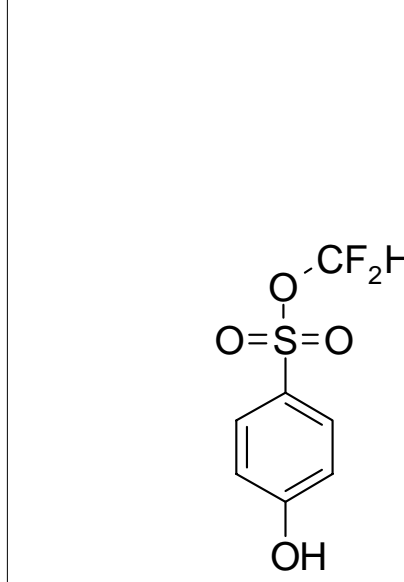

ppm (t1) 200

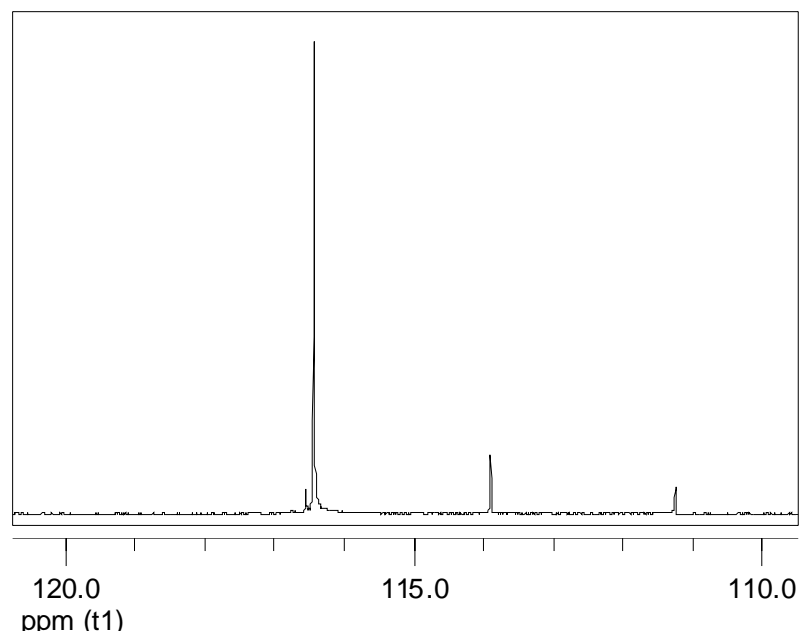

$$
\text { ppm (t1) }
$$
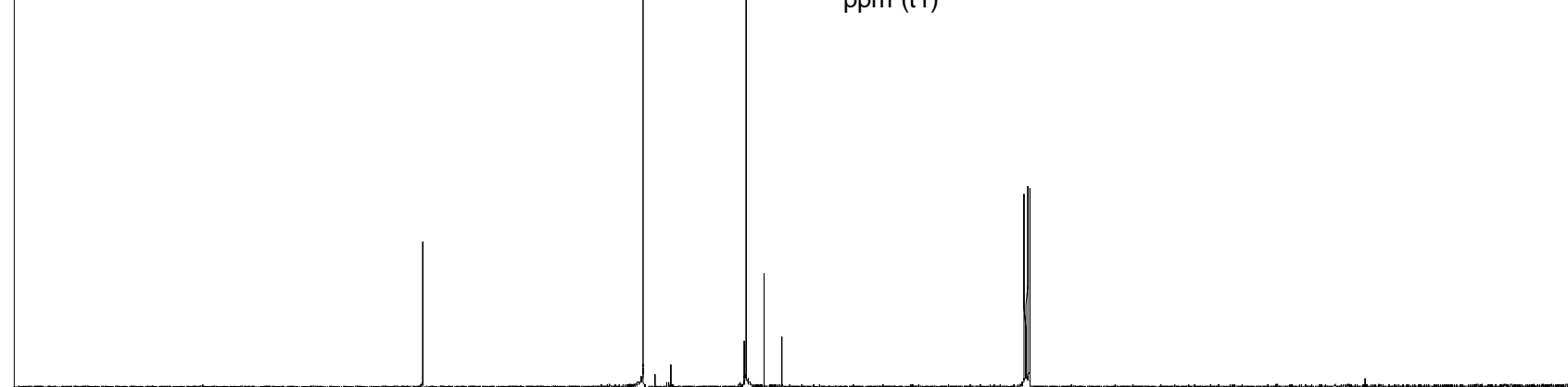


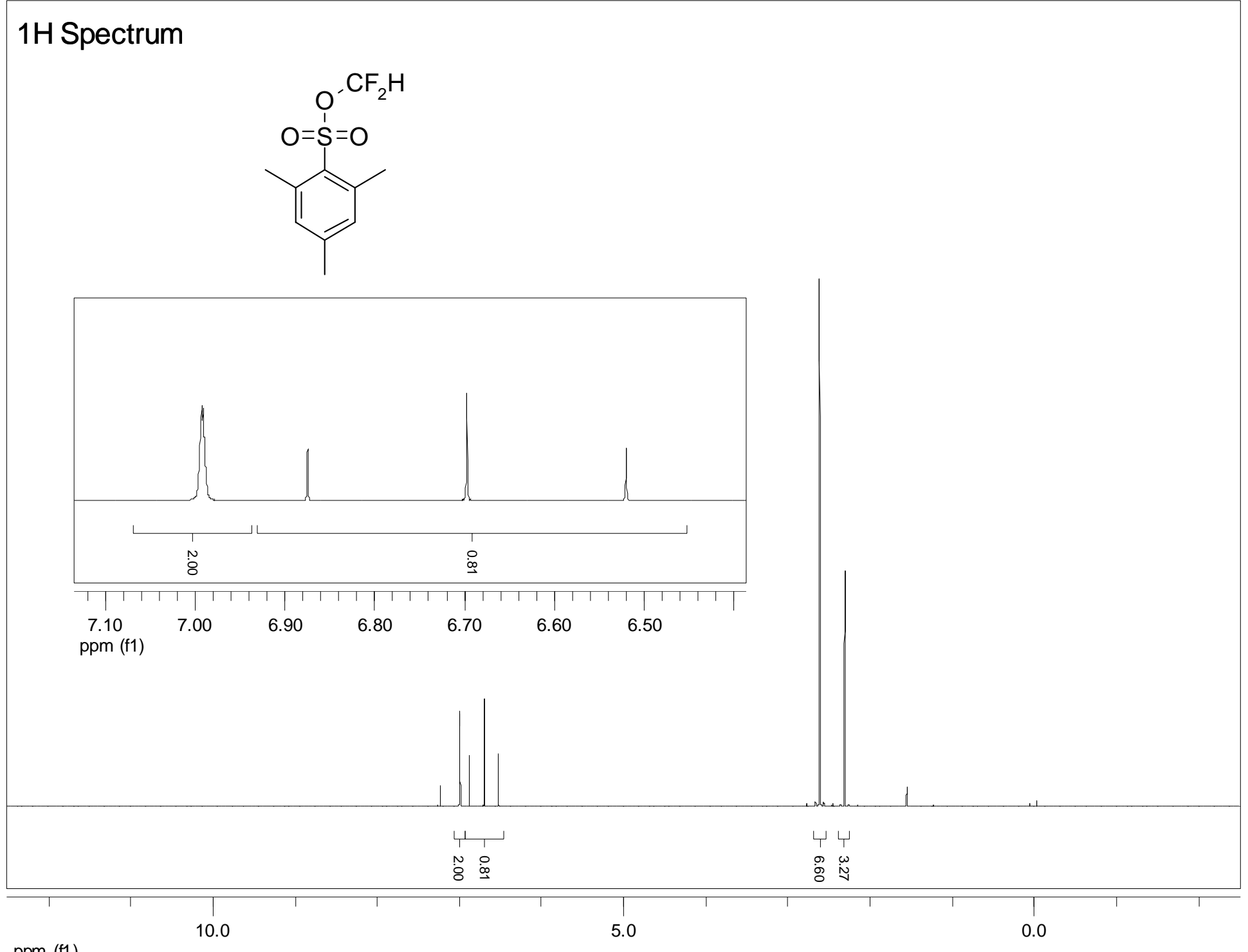

ppm (f1)

- S57 - 


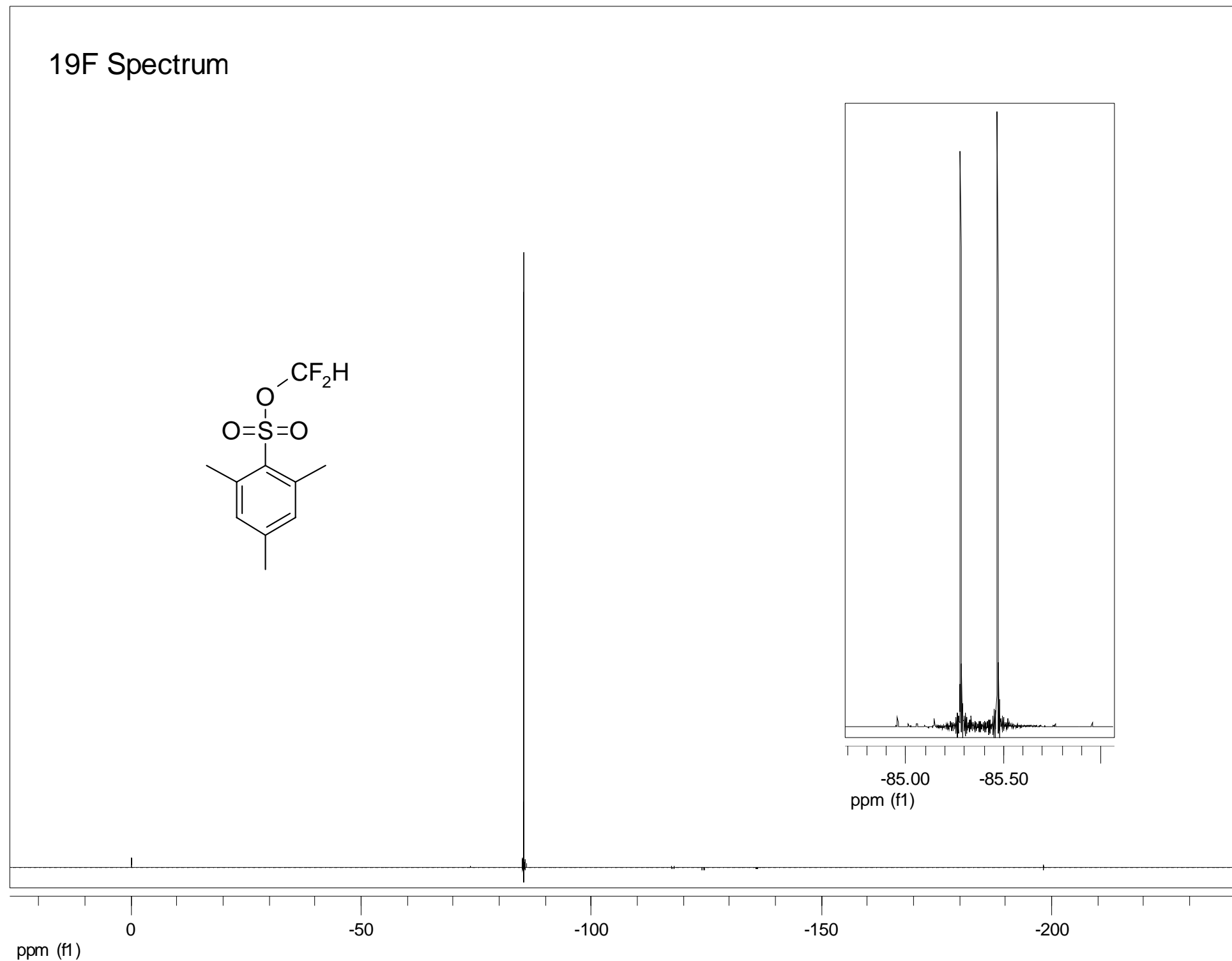

- S58 - 


\section{C Spectrum}

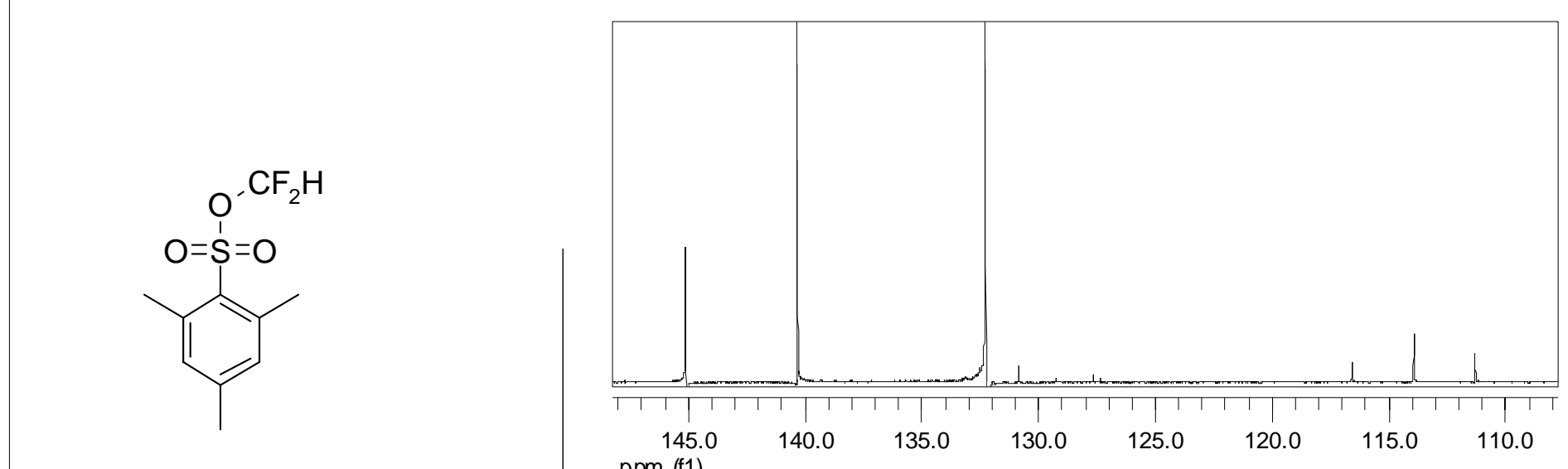

ppm (f1)

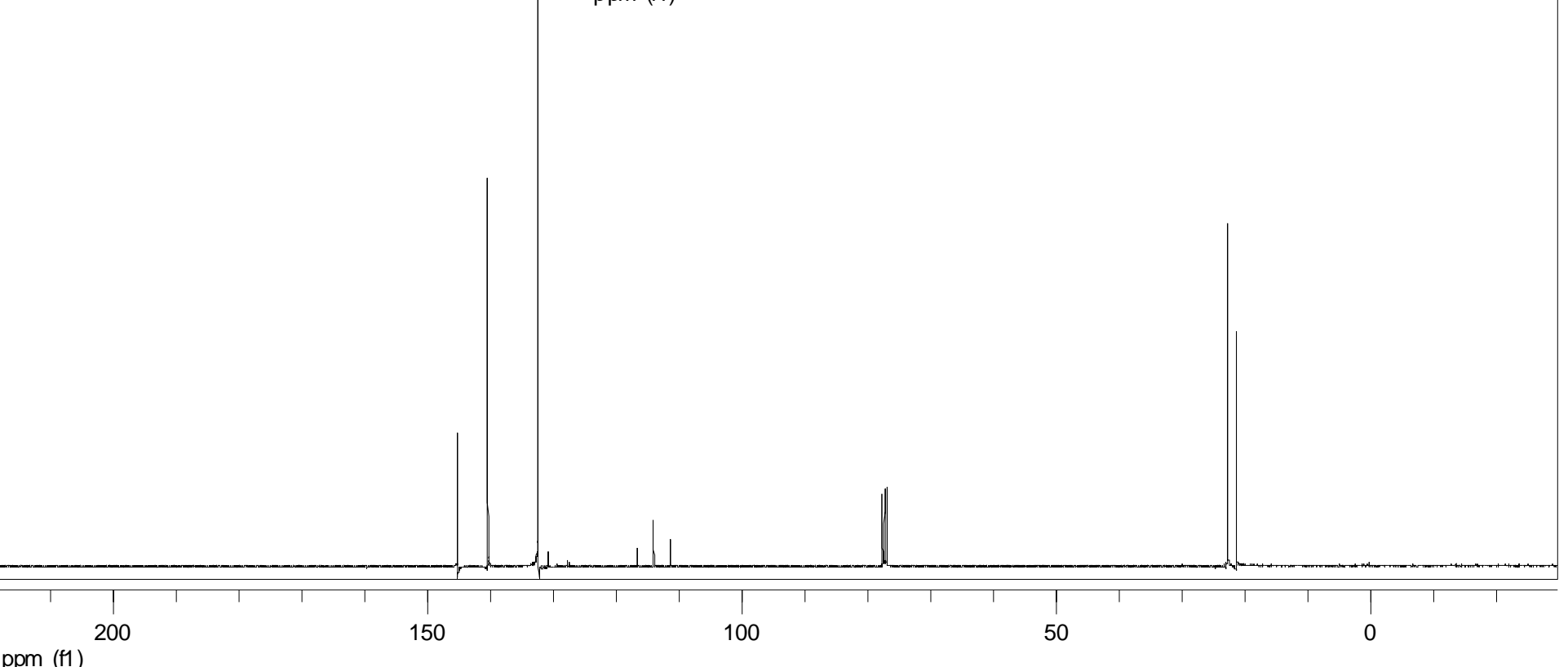

ppm (f1)

- S59 - 


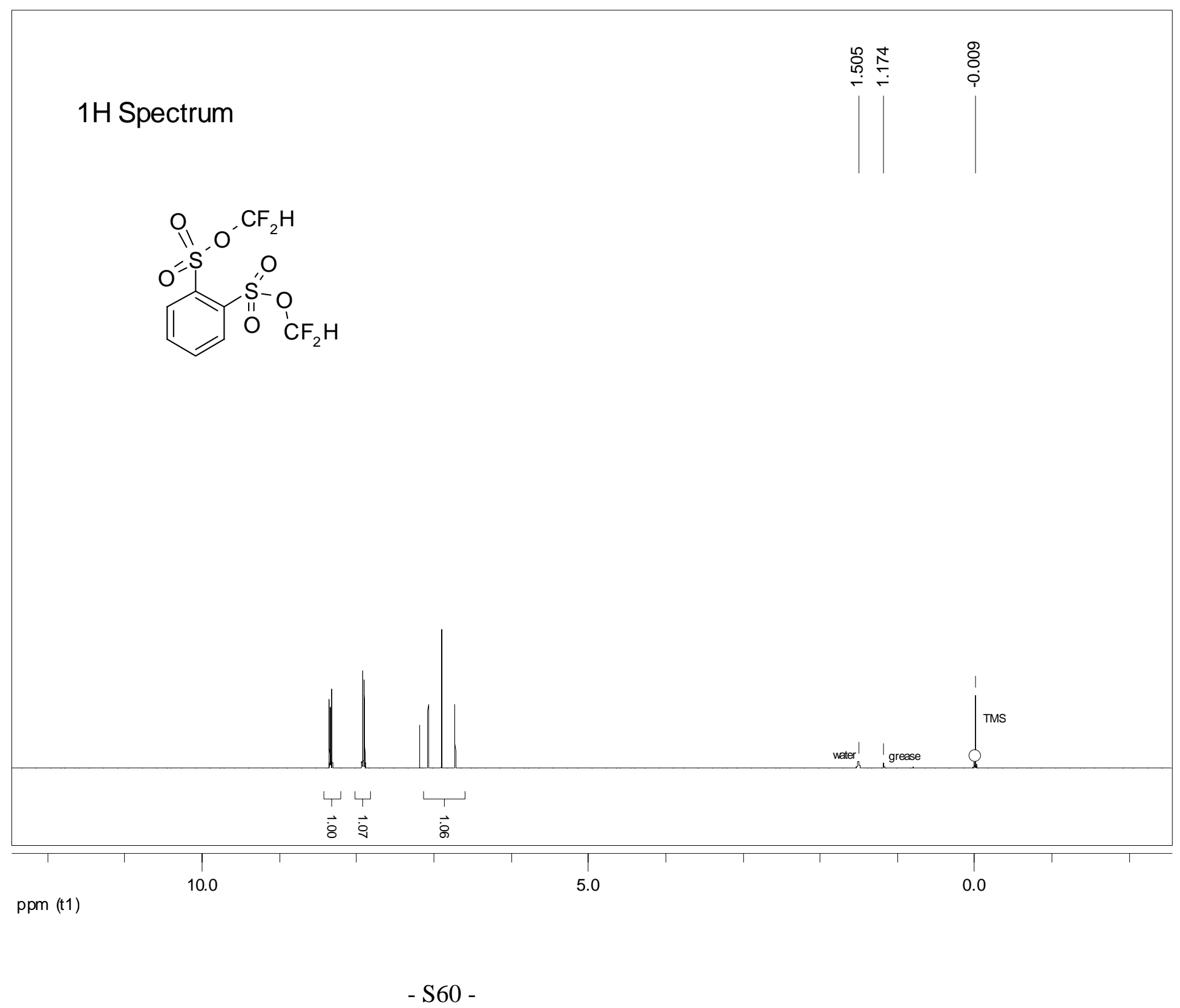




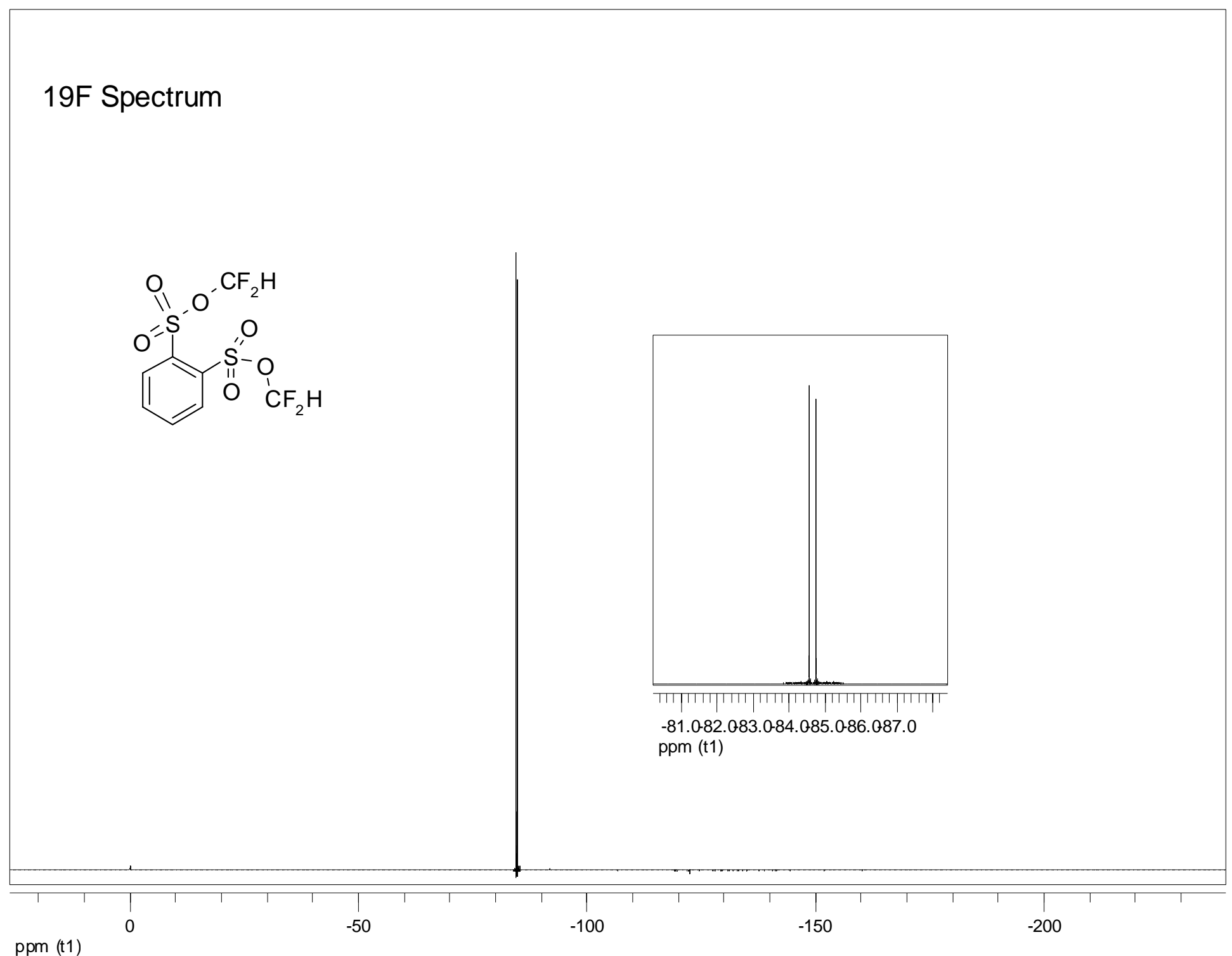

- S61 - 


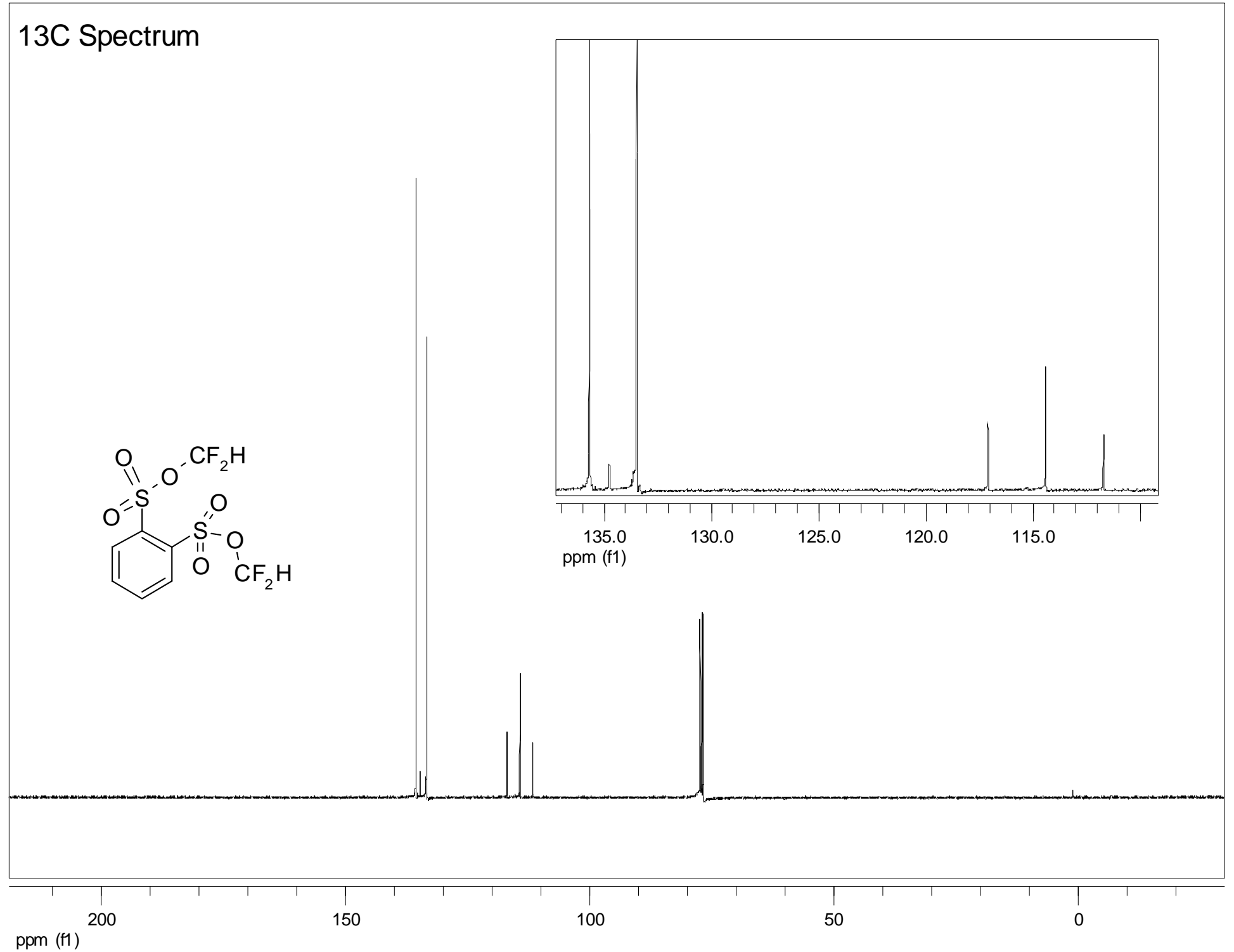

- S62 - 


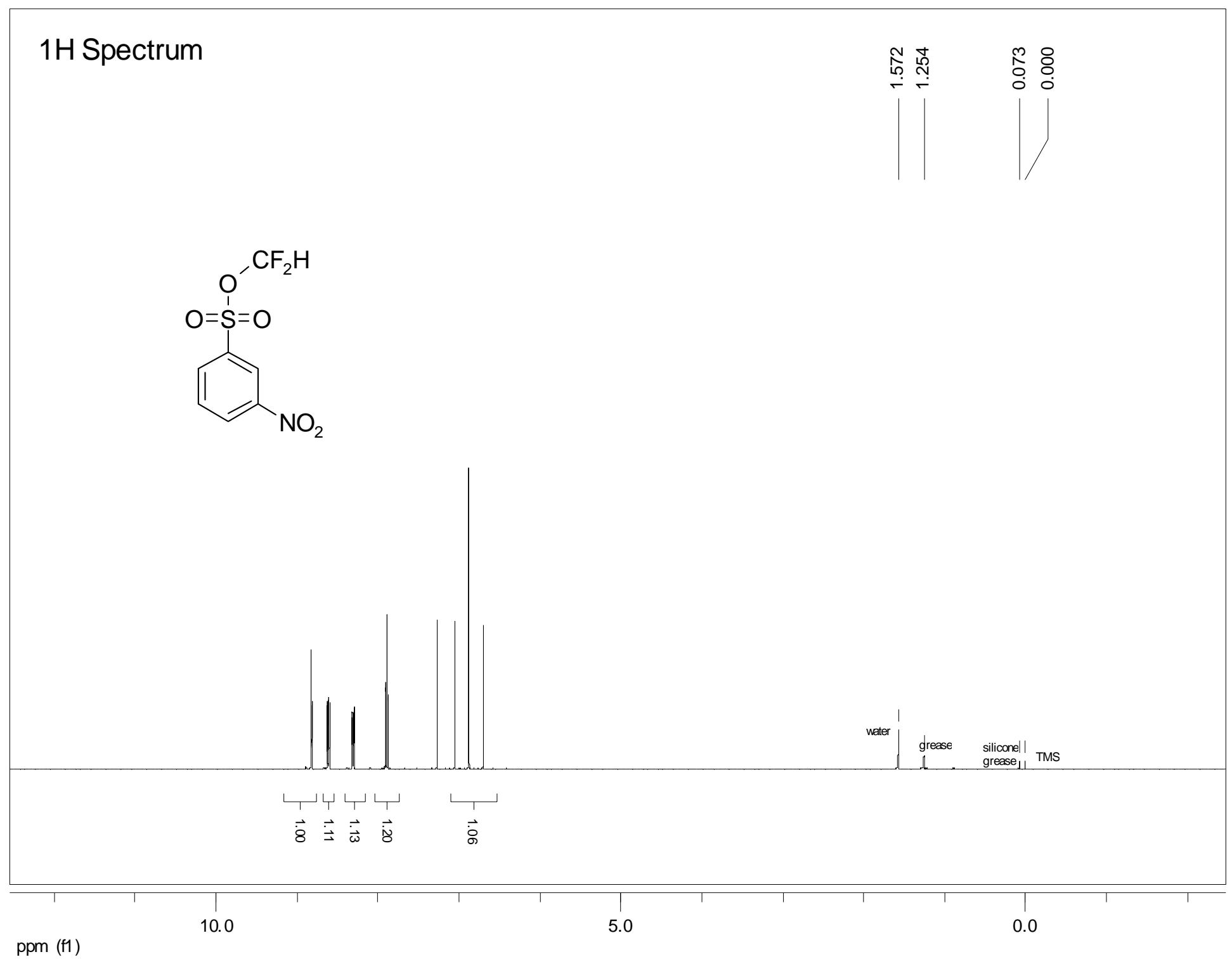

- S63 - 


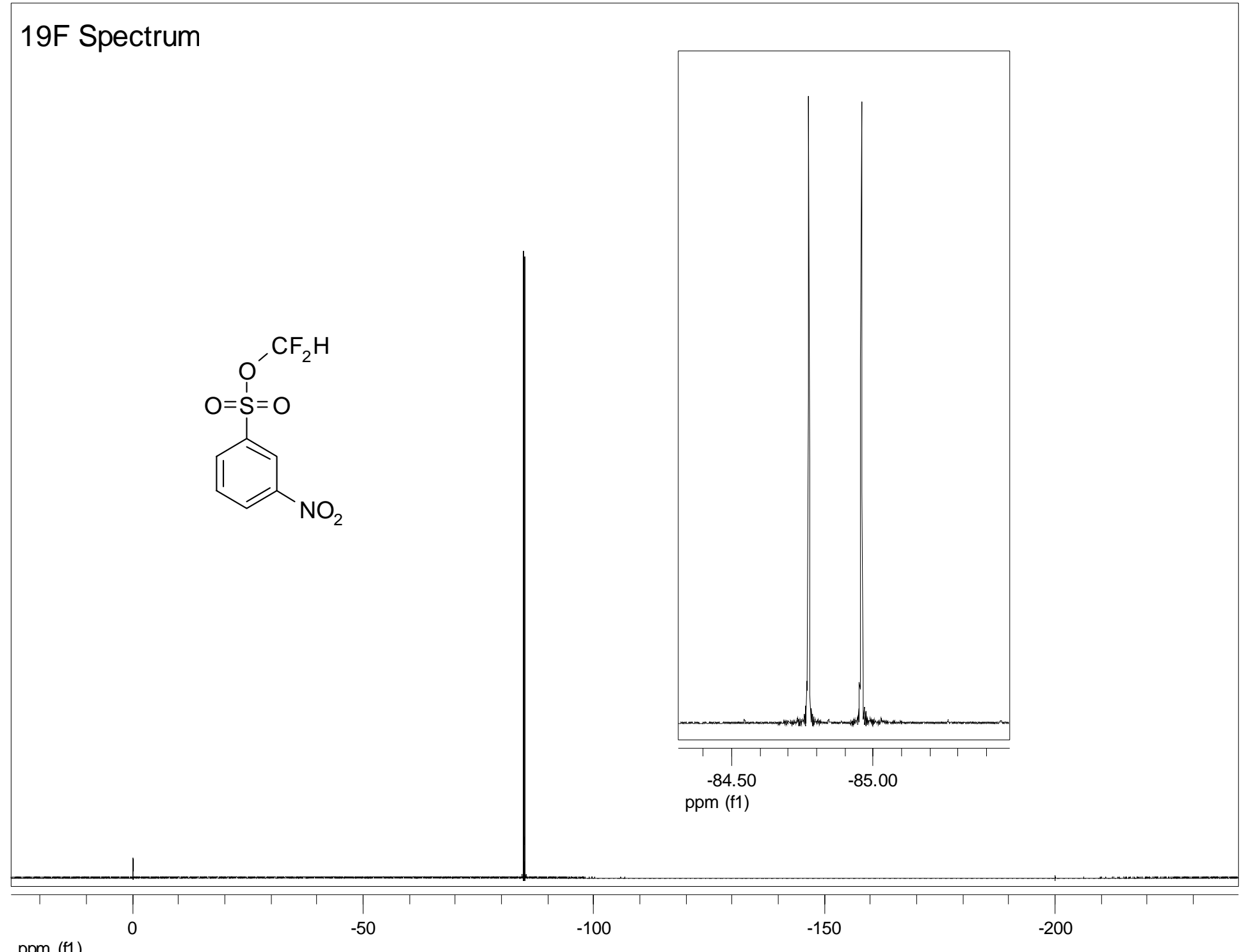

ppm (f1)

- S64 - 


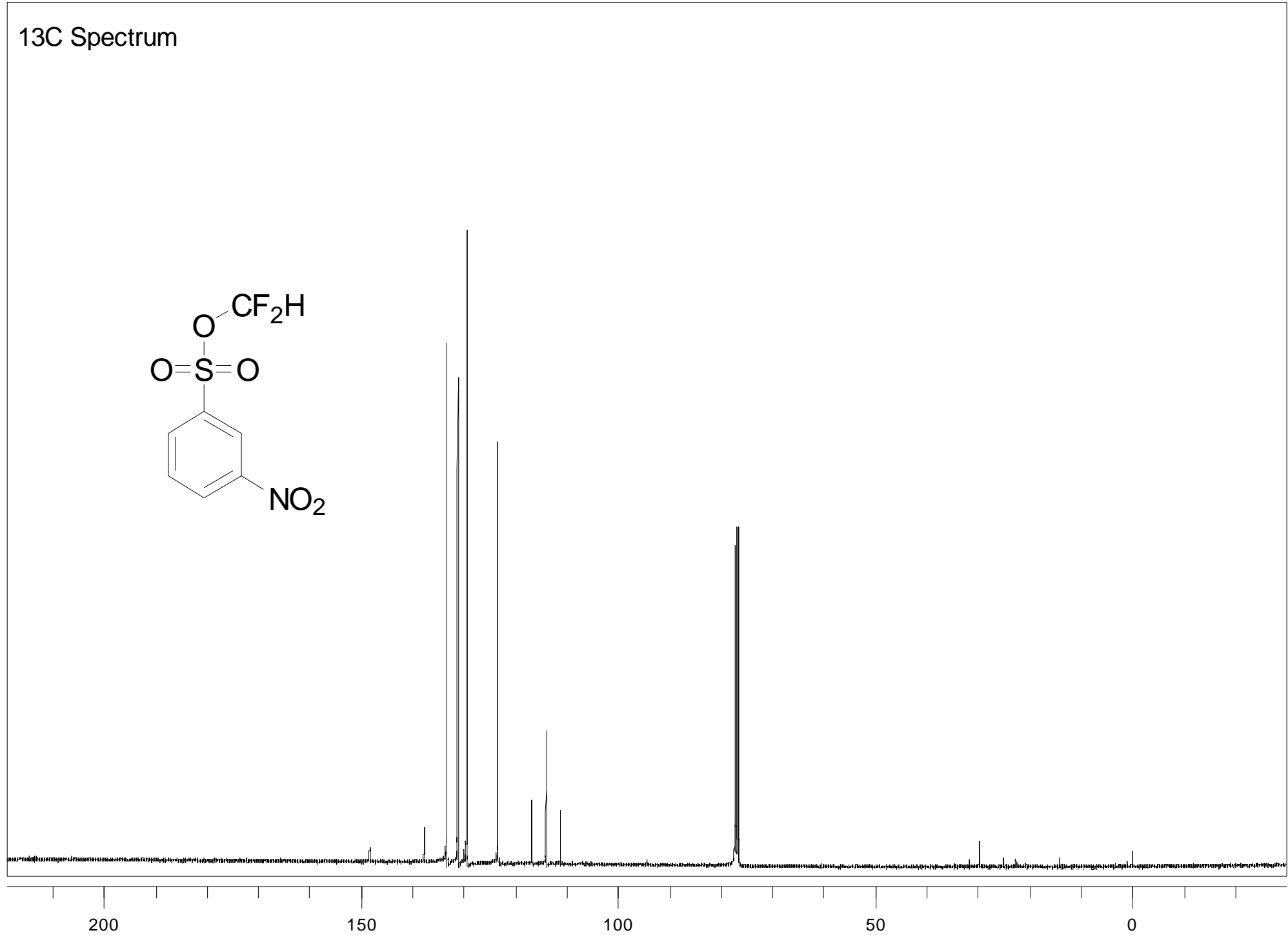

ppm (f1)

- S65 - 


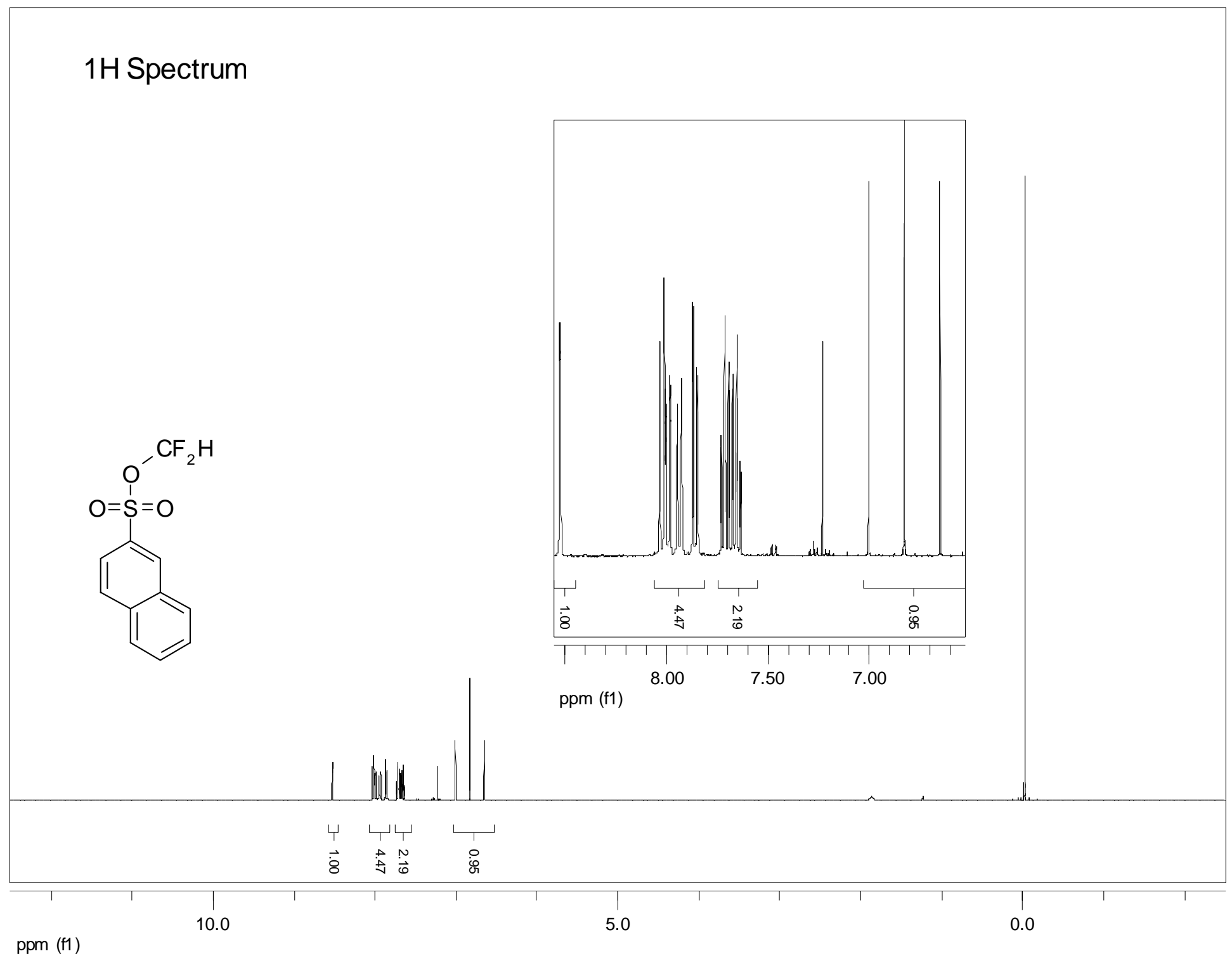

- S66 - 


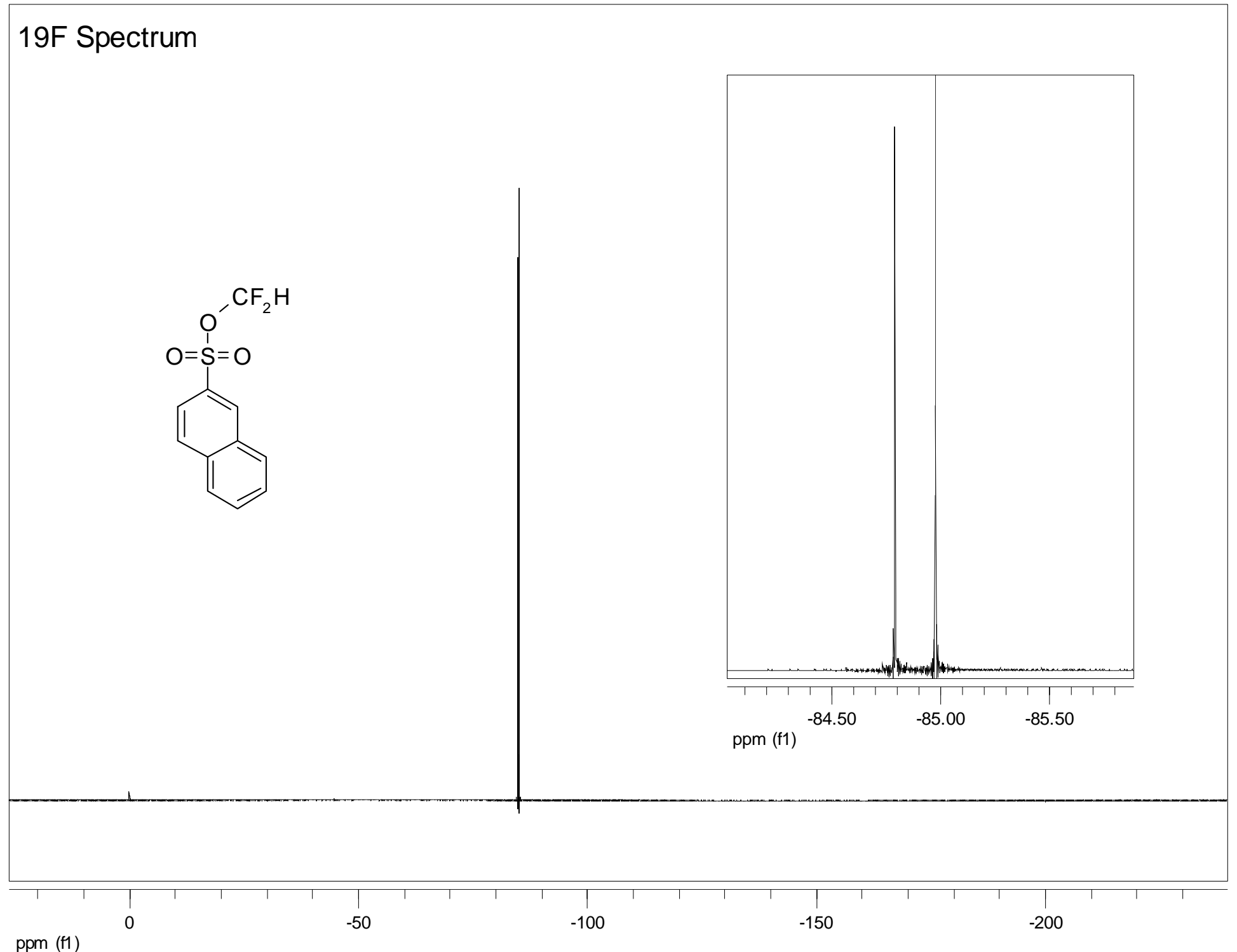

- S67 - 


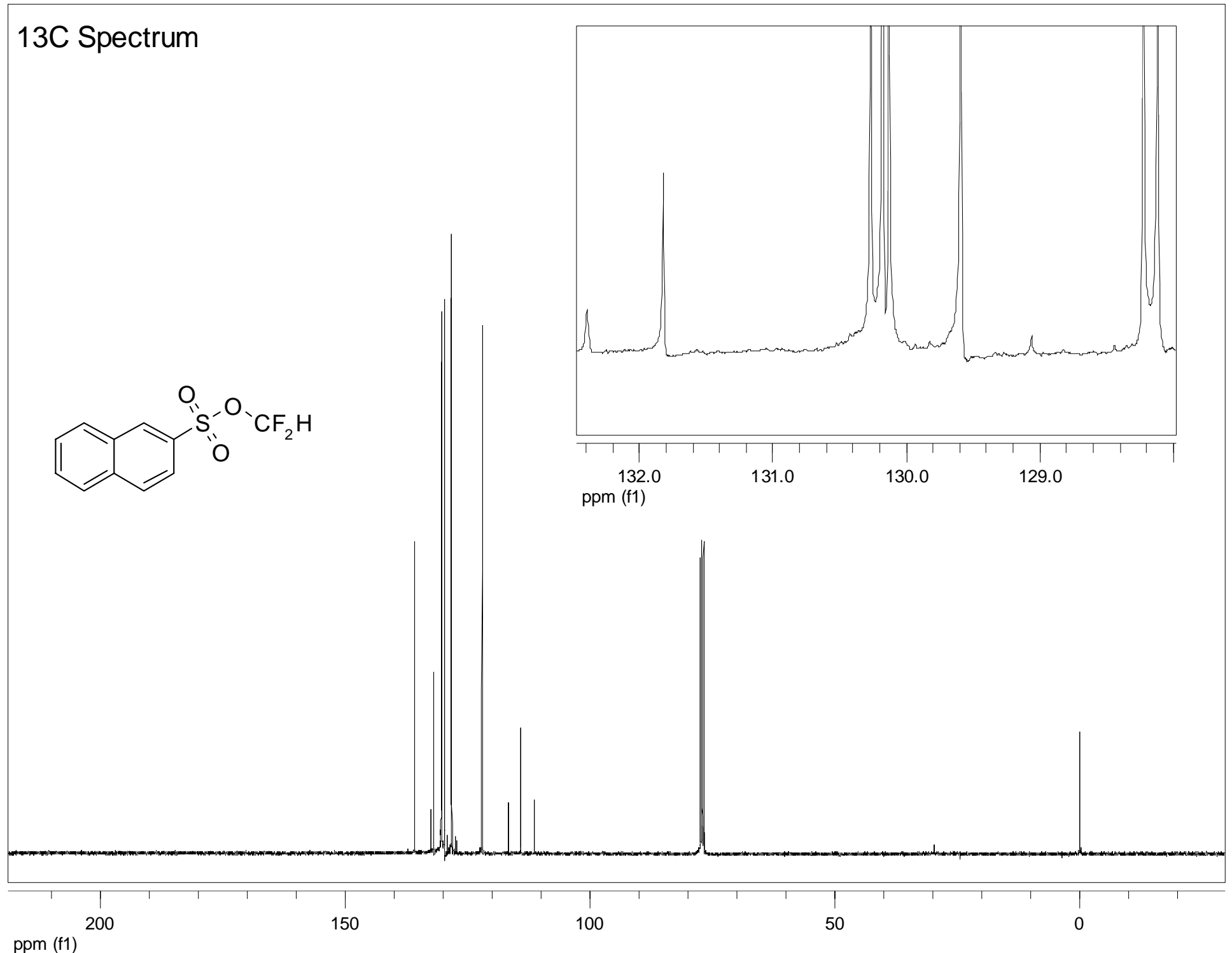

- S68 - 


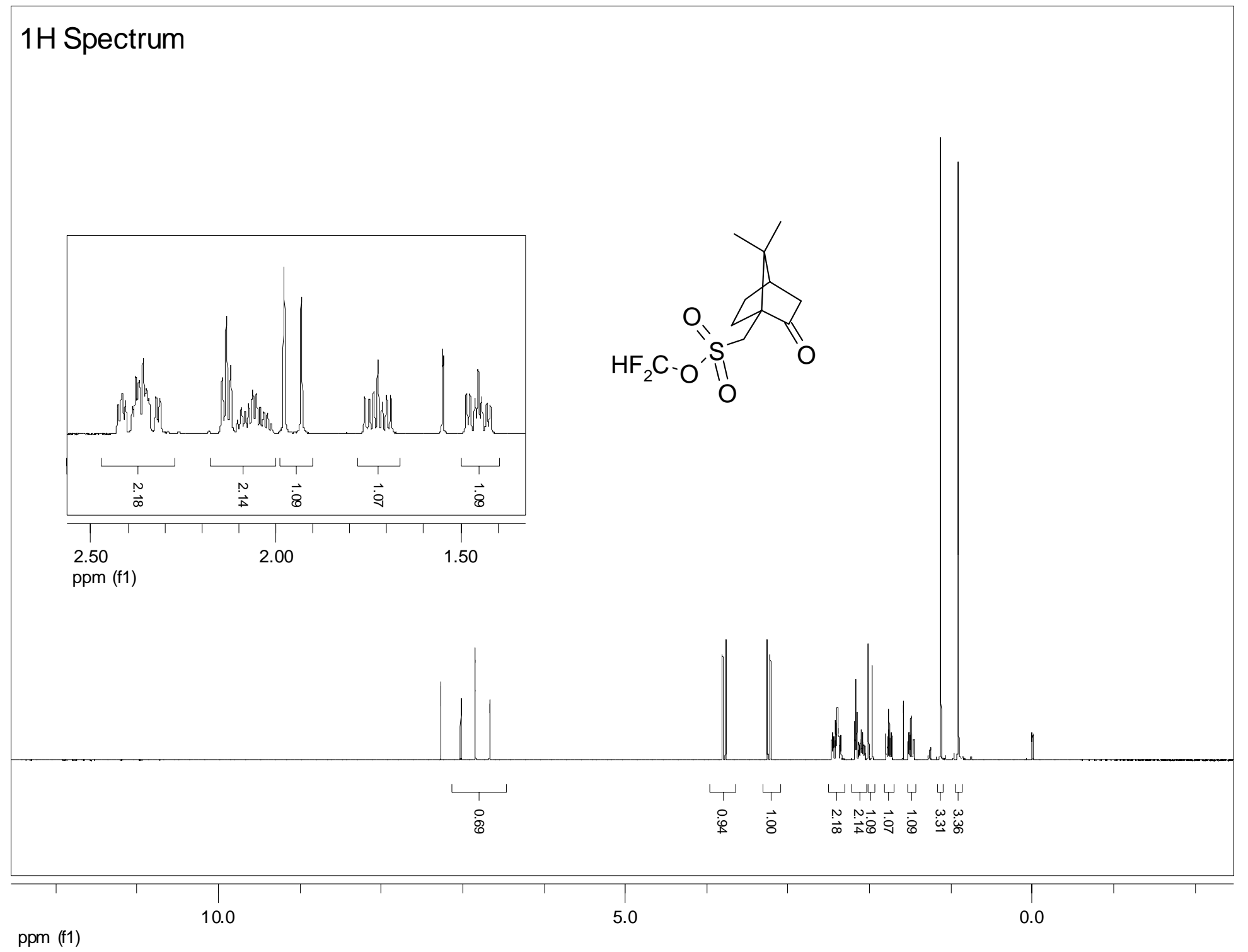

- S69 - 


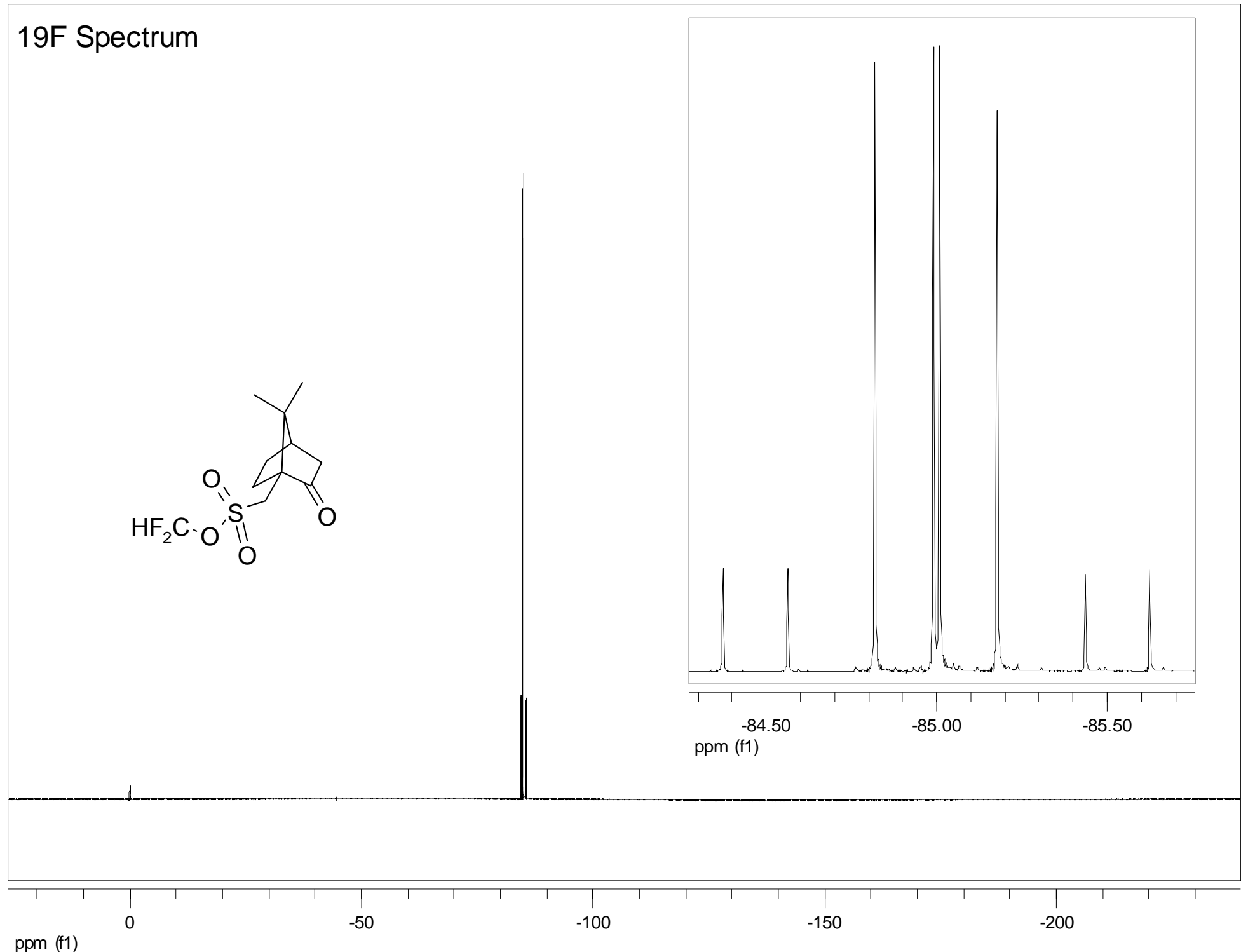

- S70 - 


\section{C Spectrum}

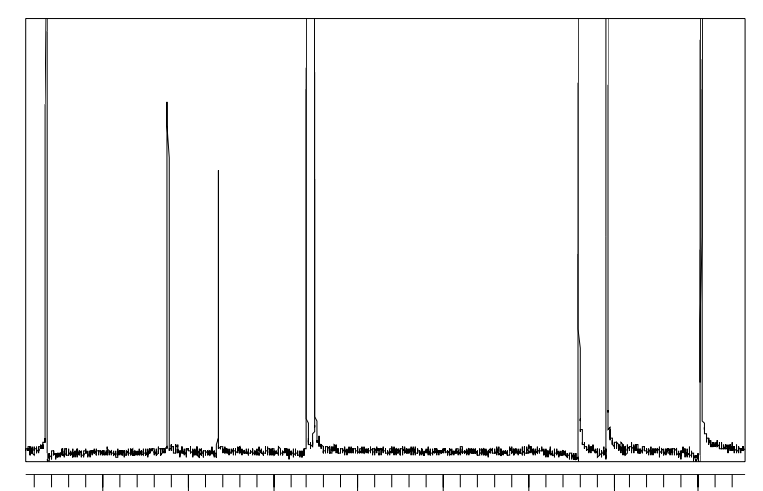

$\mathrm{HF}^{2} \mathrm{C}-\mathrm{O}-\mathrm{S}$

$\begin{array}{llllllll}55.0 & 50.0 & 45.0 & 40.0 & 35.0 & 30.0 & 25.0 & 20.0\end{array}$ ppm (f1)

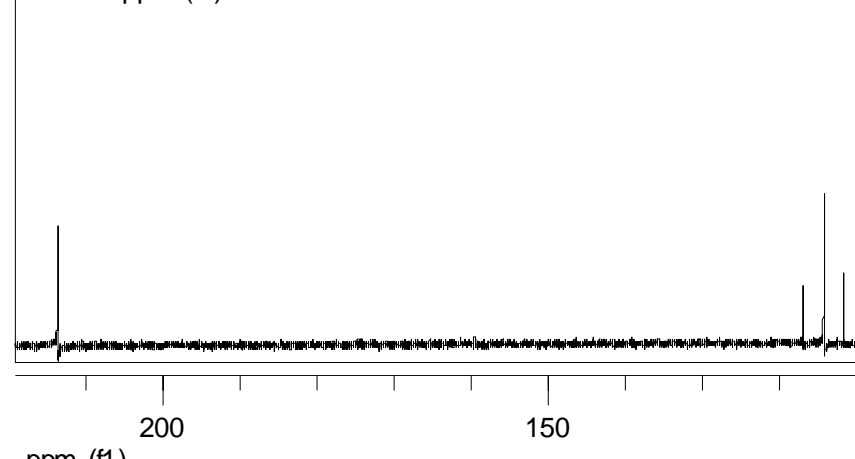

ppm (f1)
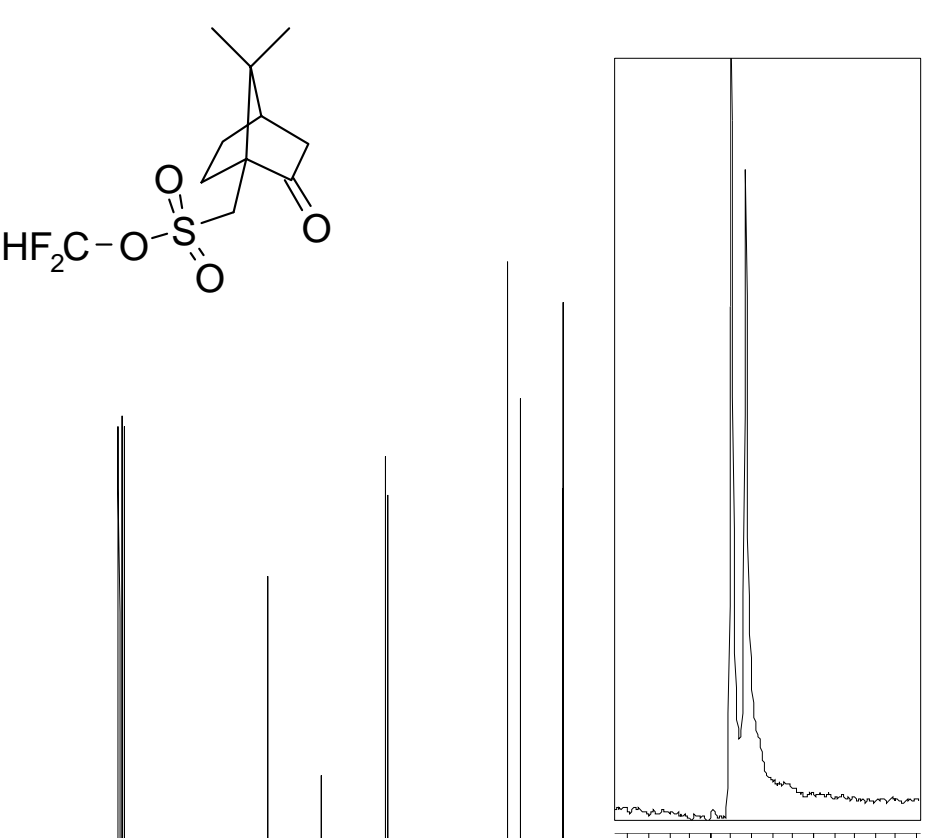

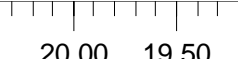
ppm (f1) 


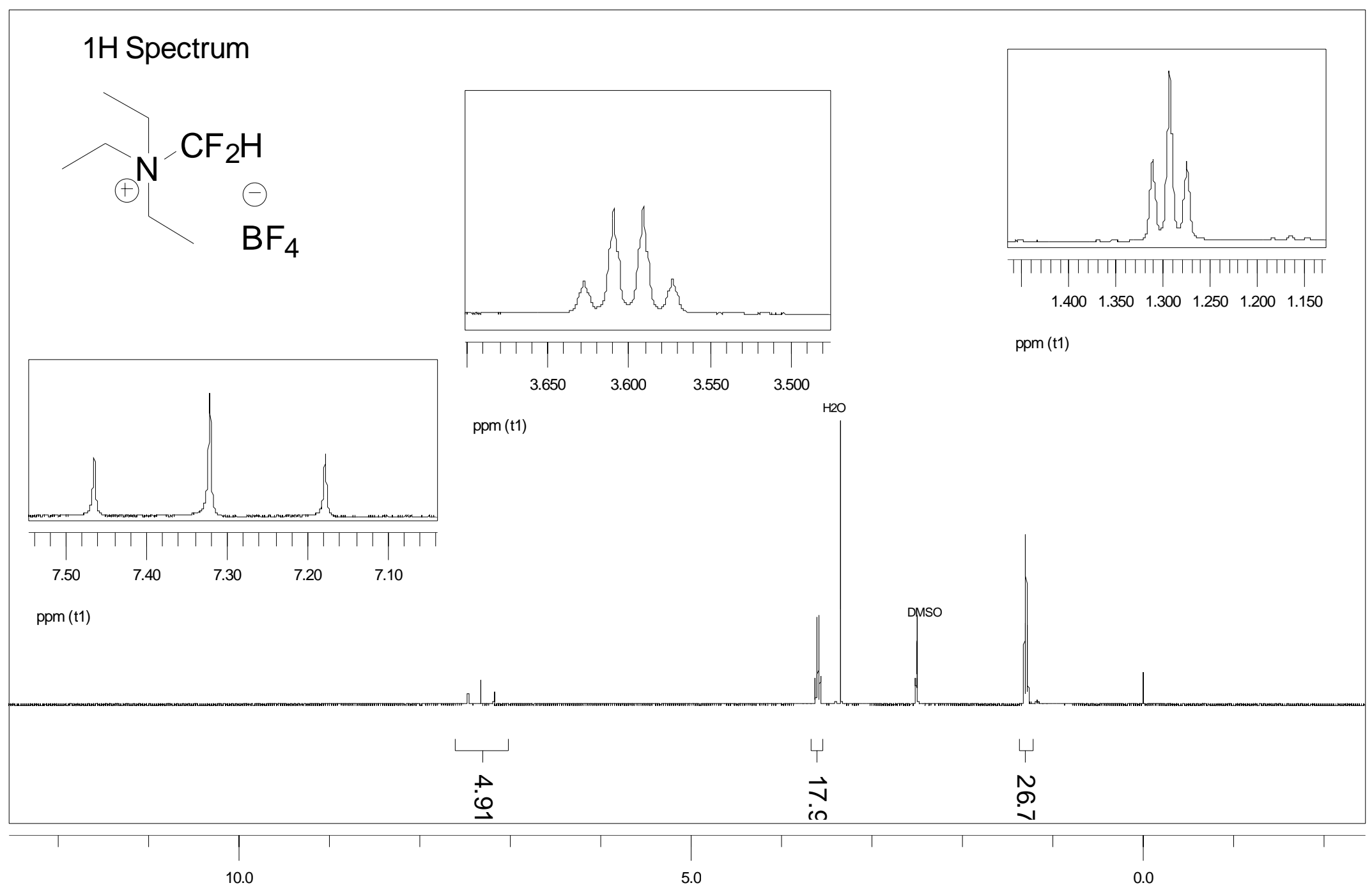

ppm (t1) 


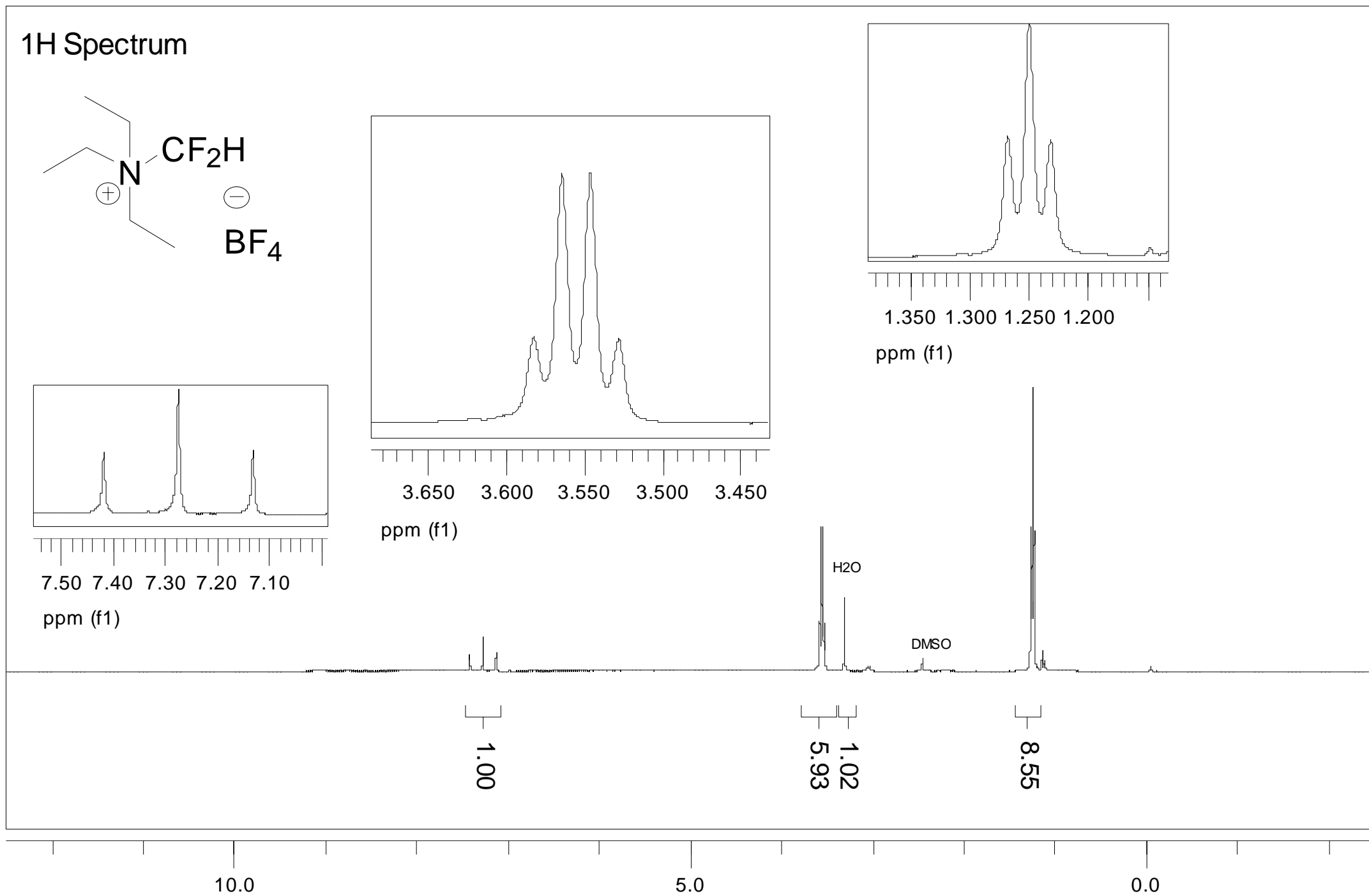

ppm (f1) 
19F Spectrum
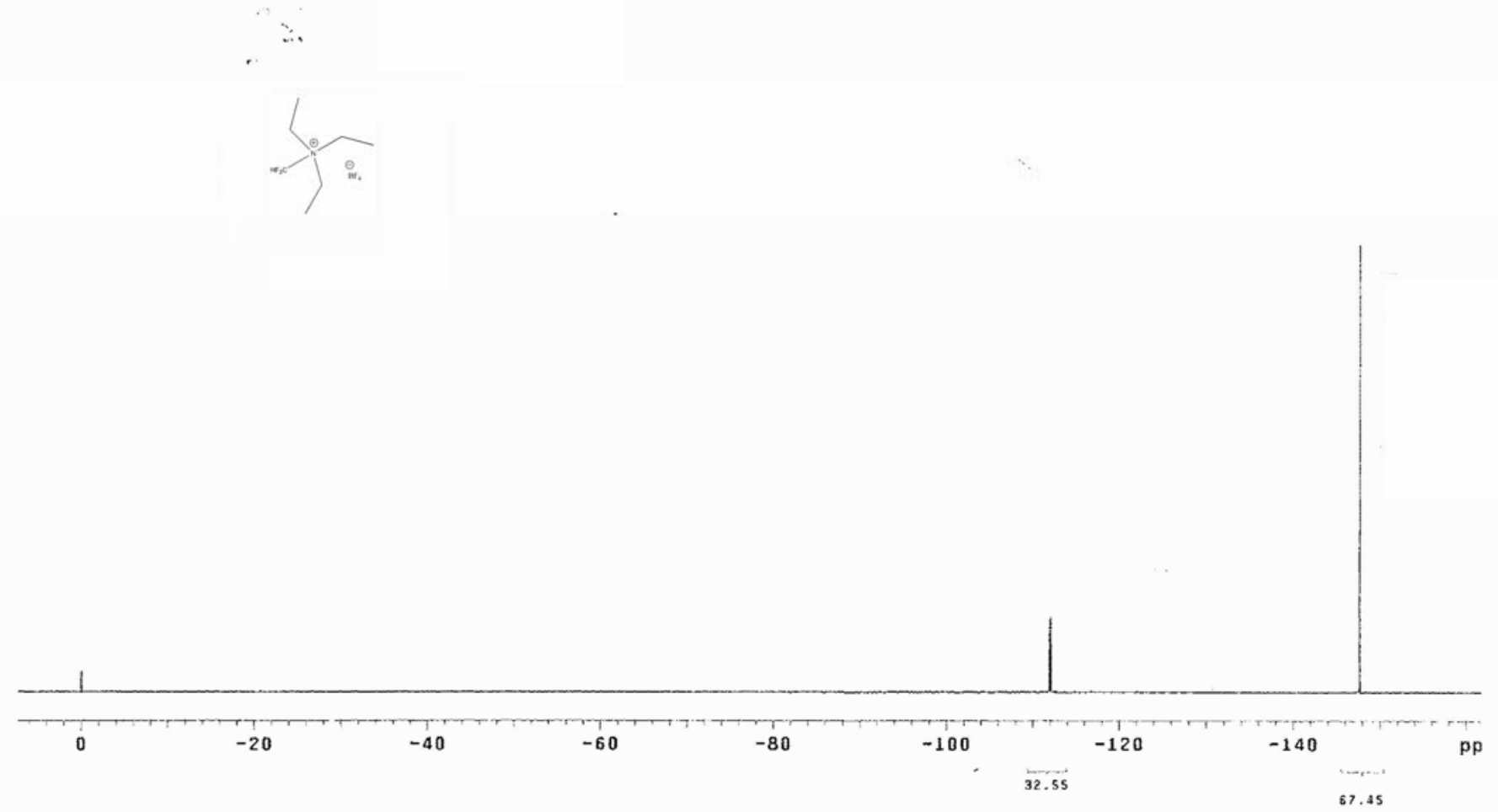

- S74 - 
19F Spectrum
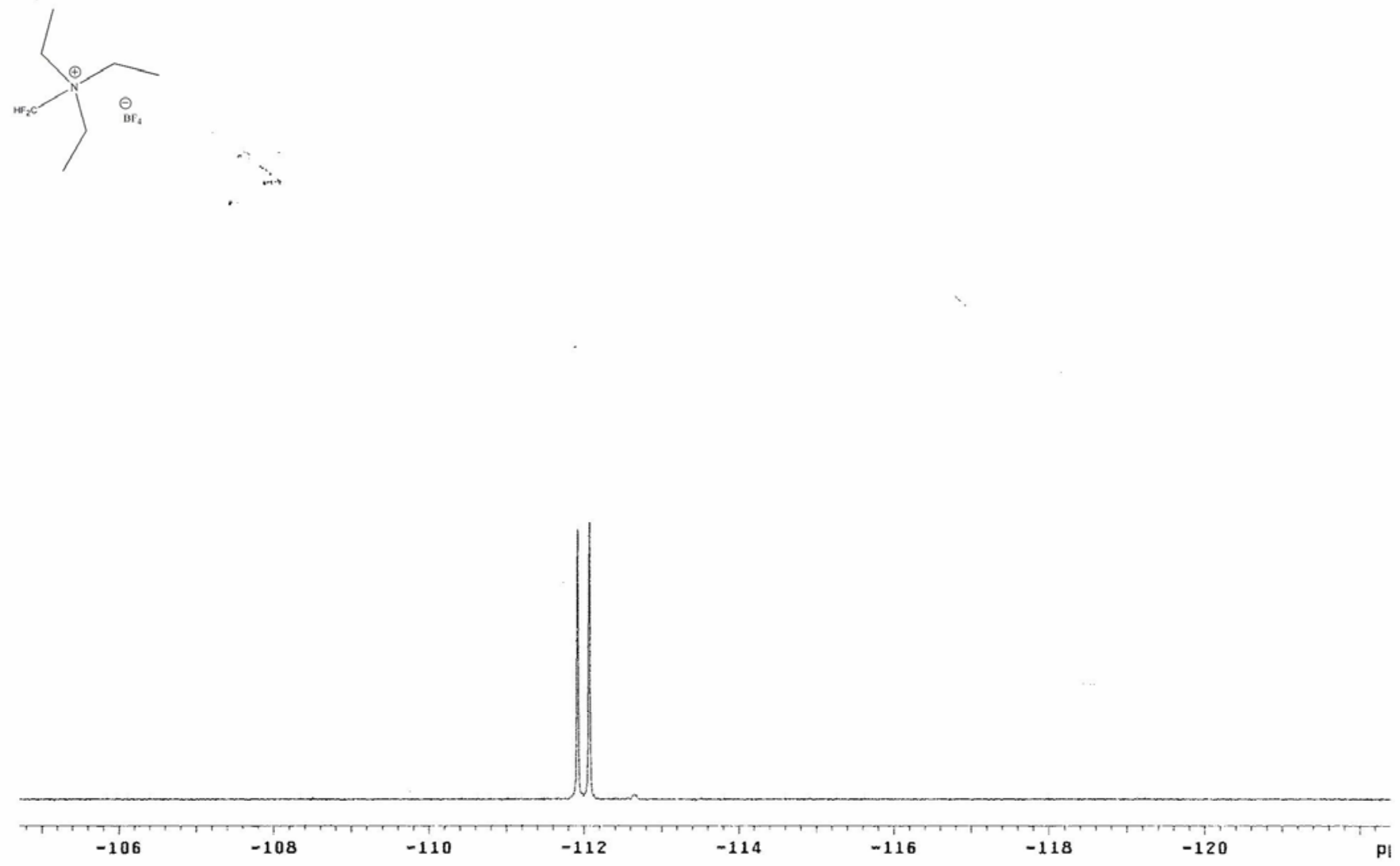

- S75 - 
$19 F$ Spectrum
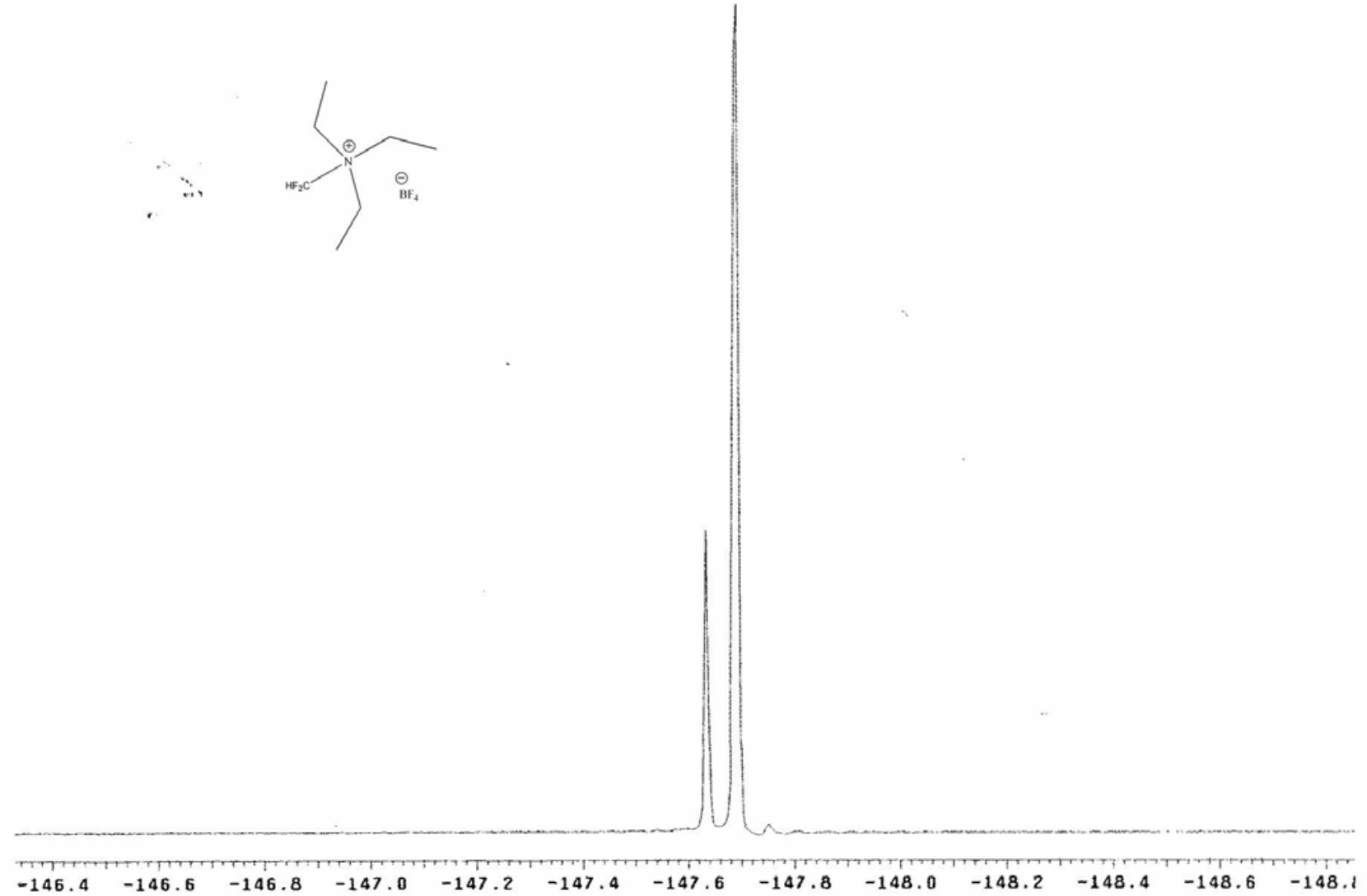

- S76 - 


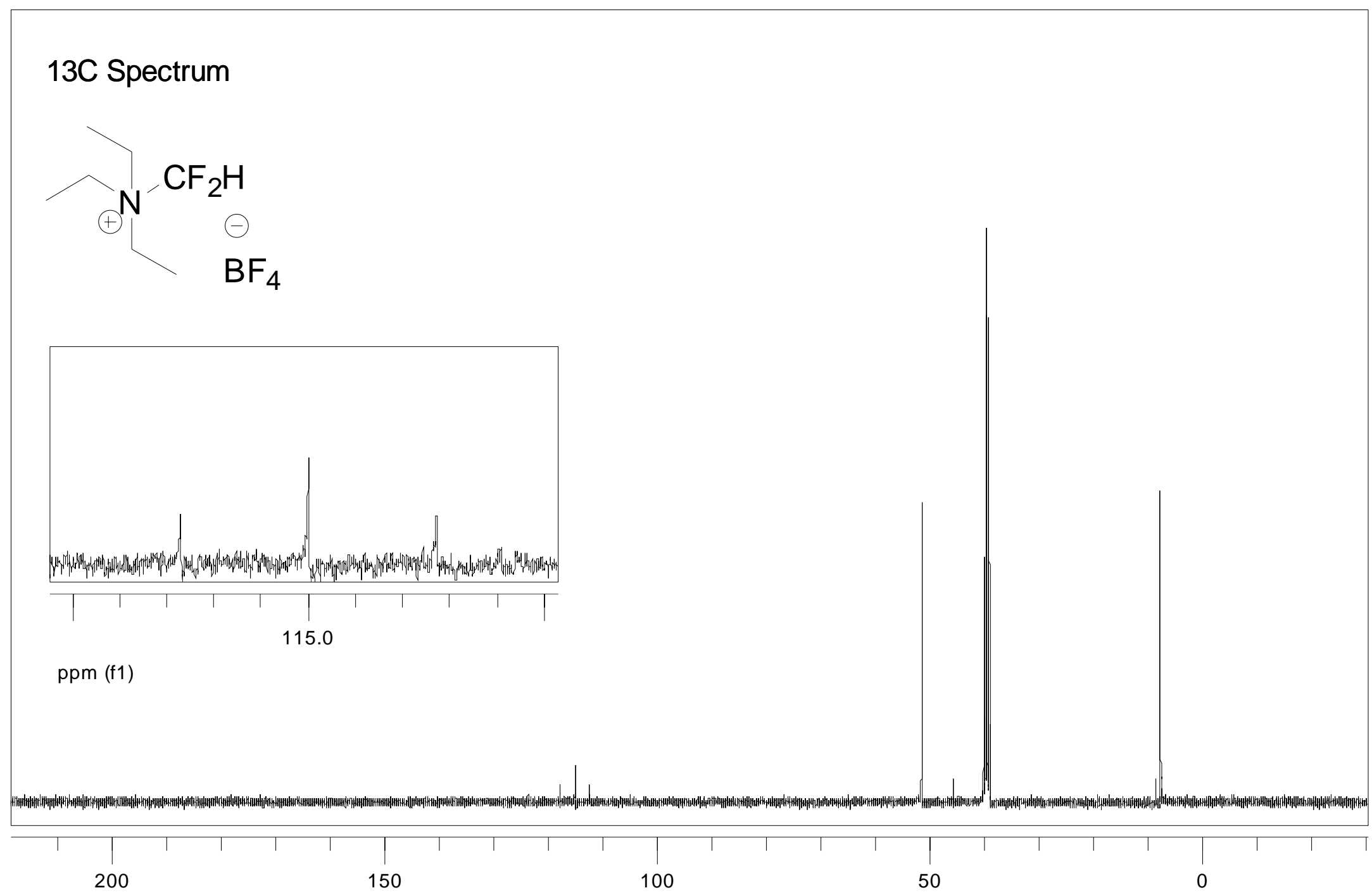

ppm (f1) 


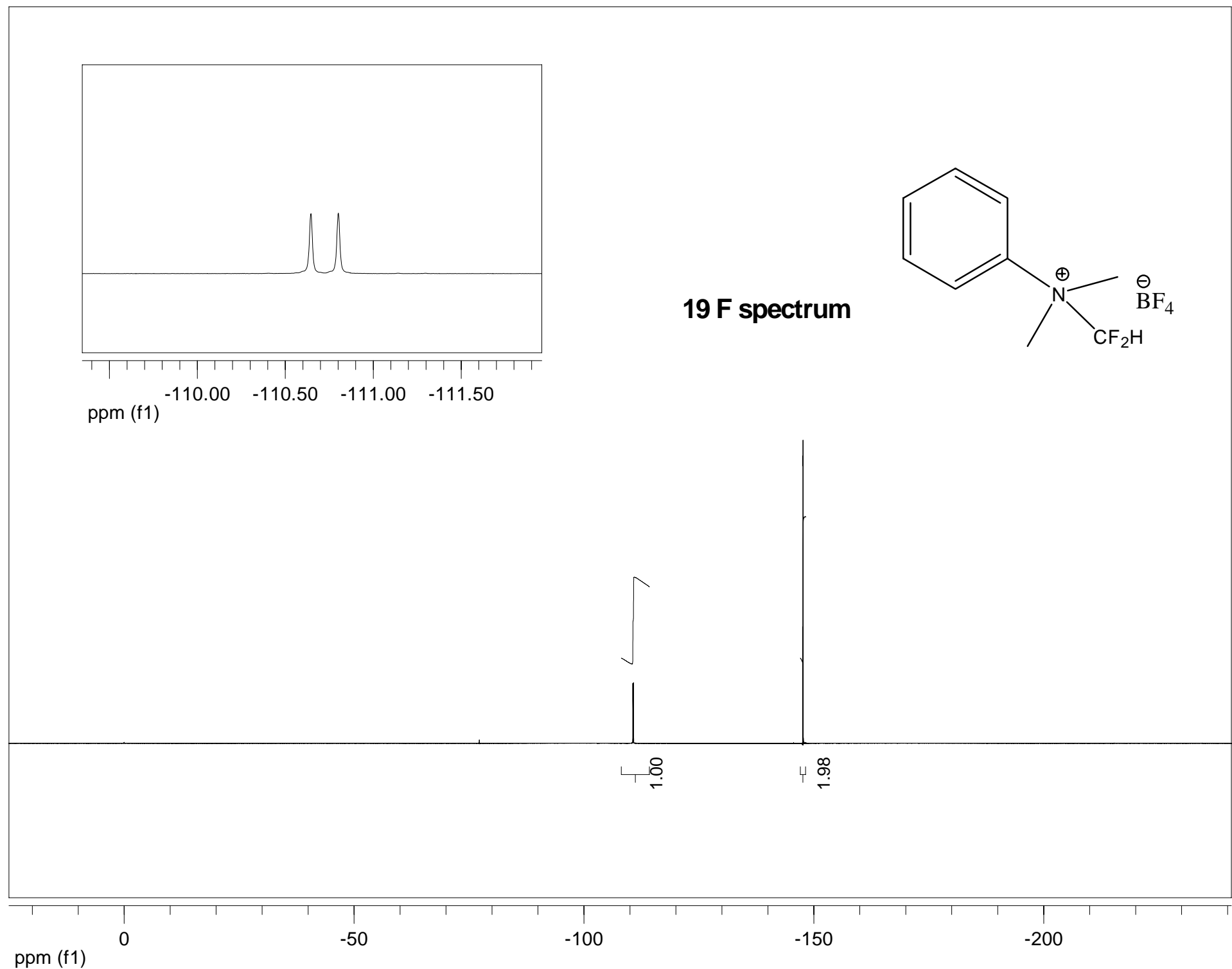

- S78 - 


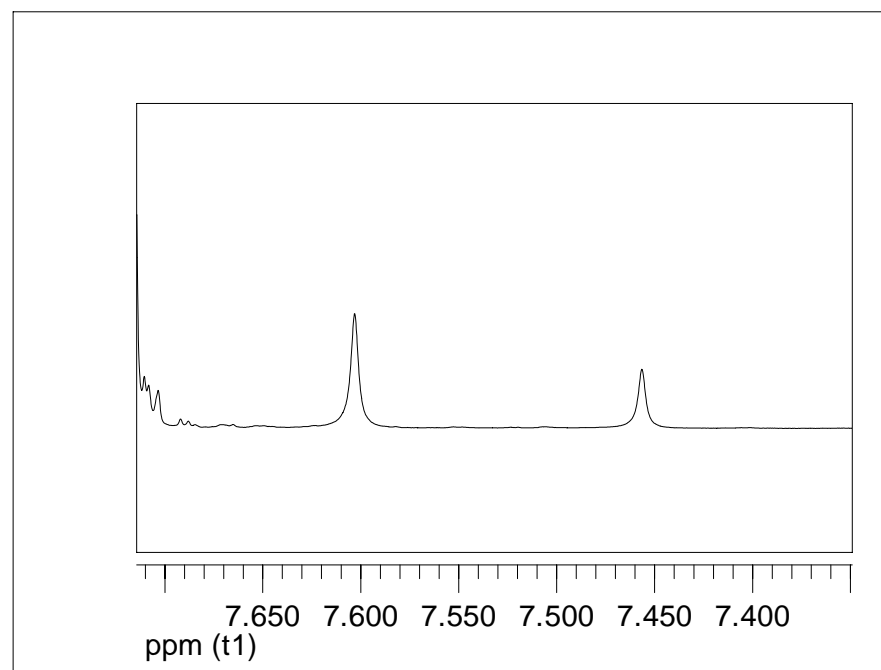

\section{H spectrum}
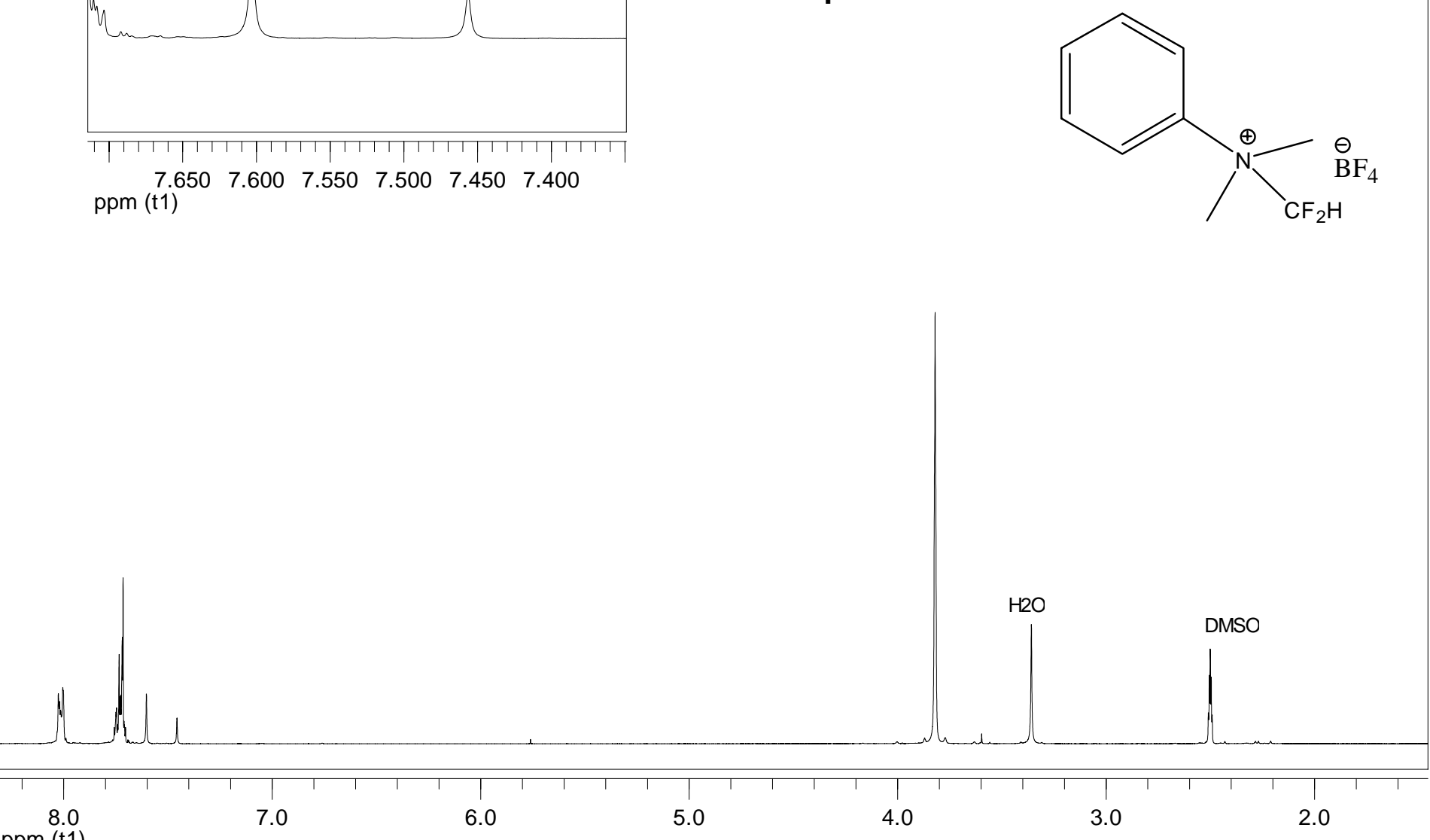

ppm (t1) 


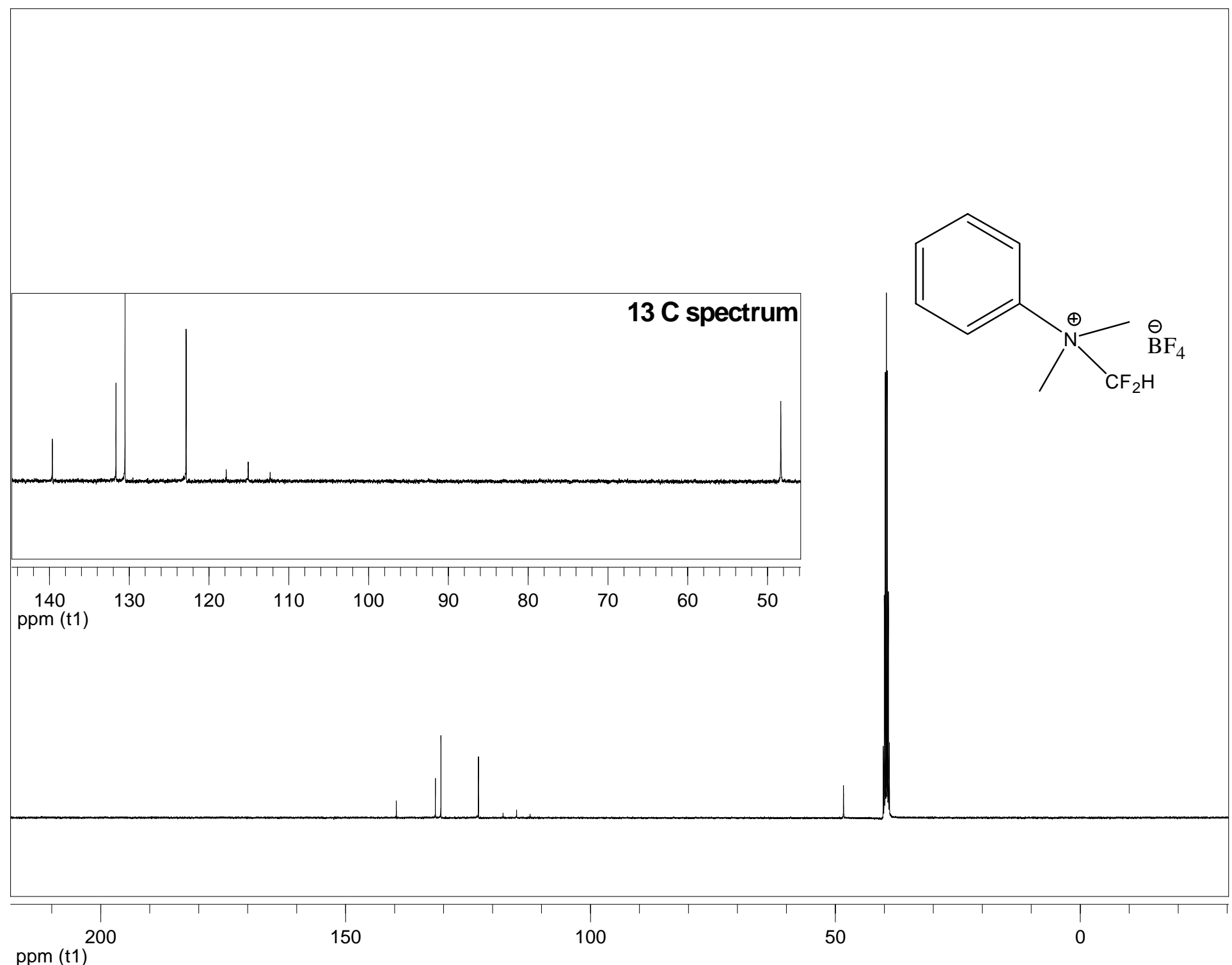

- S80 - 
$19 \mathrm{~F}$ spectrum
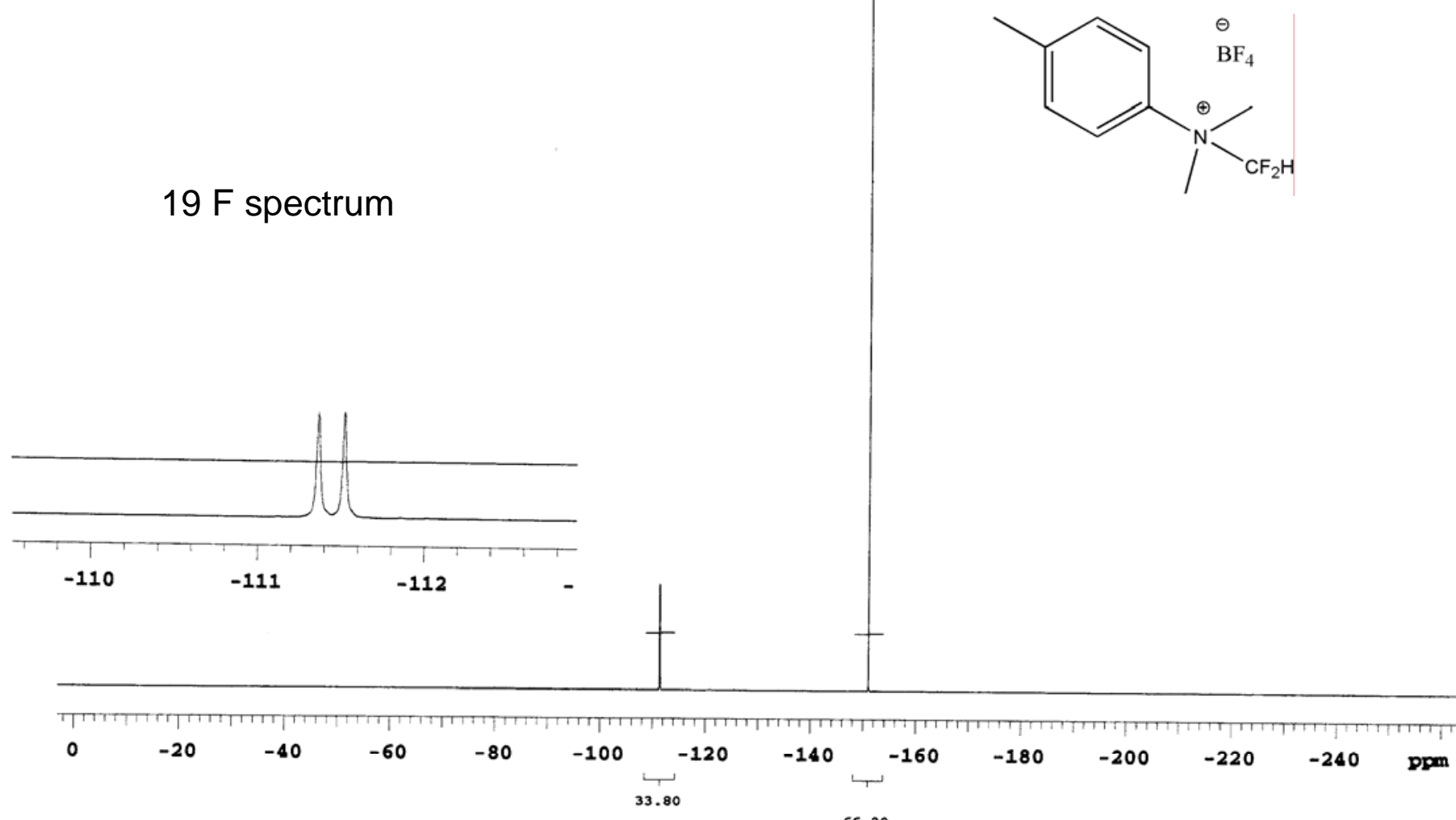


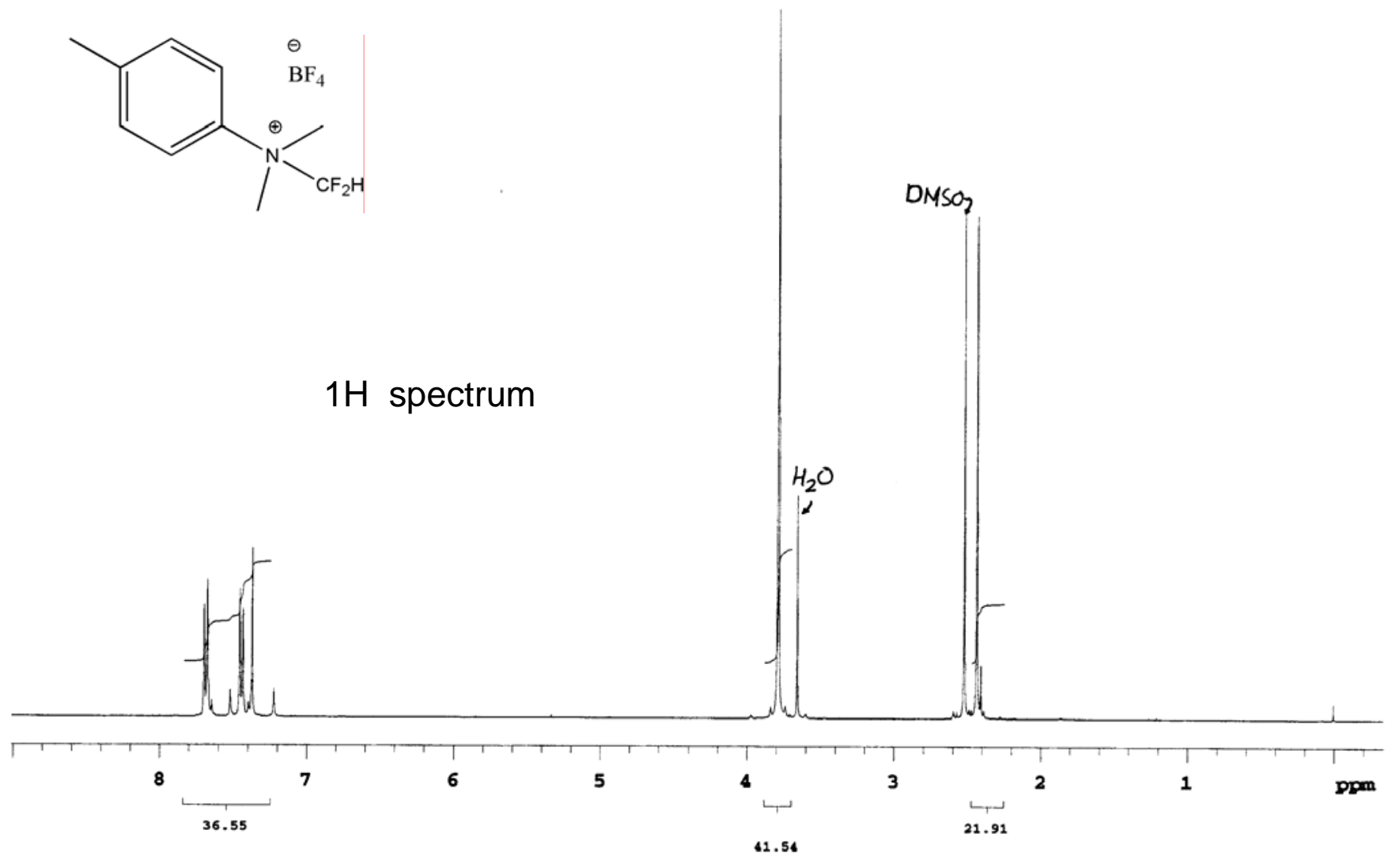

- S82 - 


\section{$13 \mathrm{C}$ spectrum}
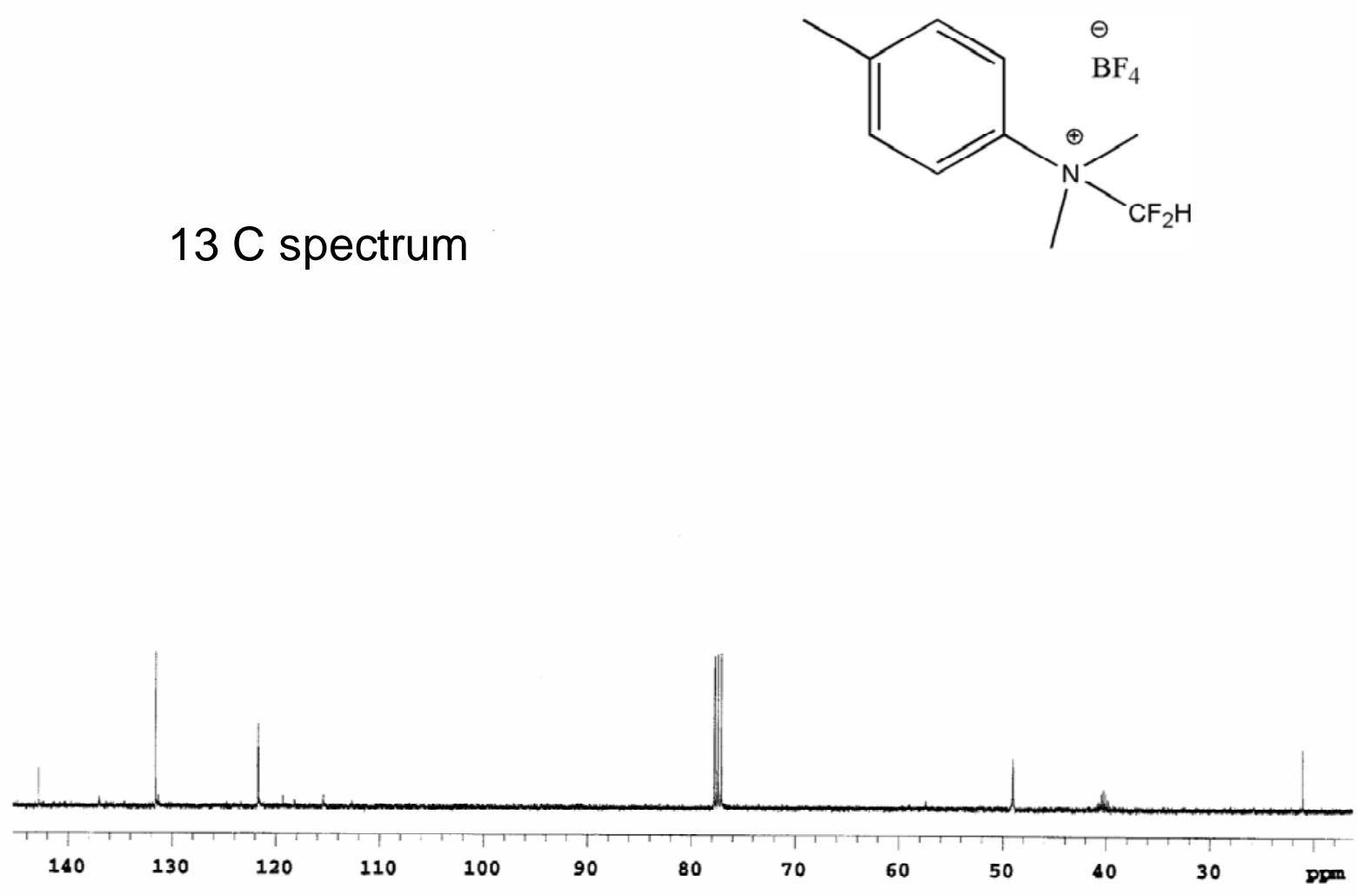


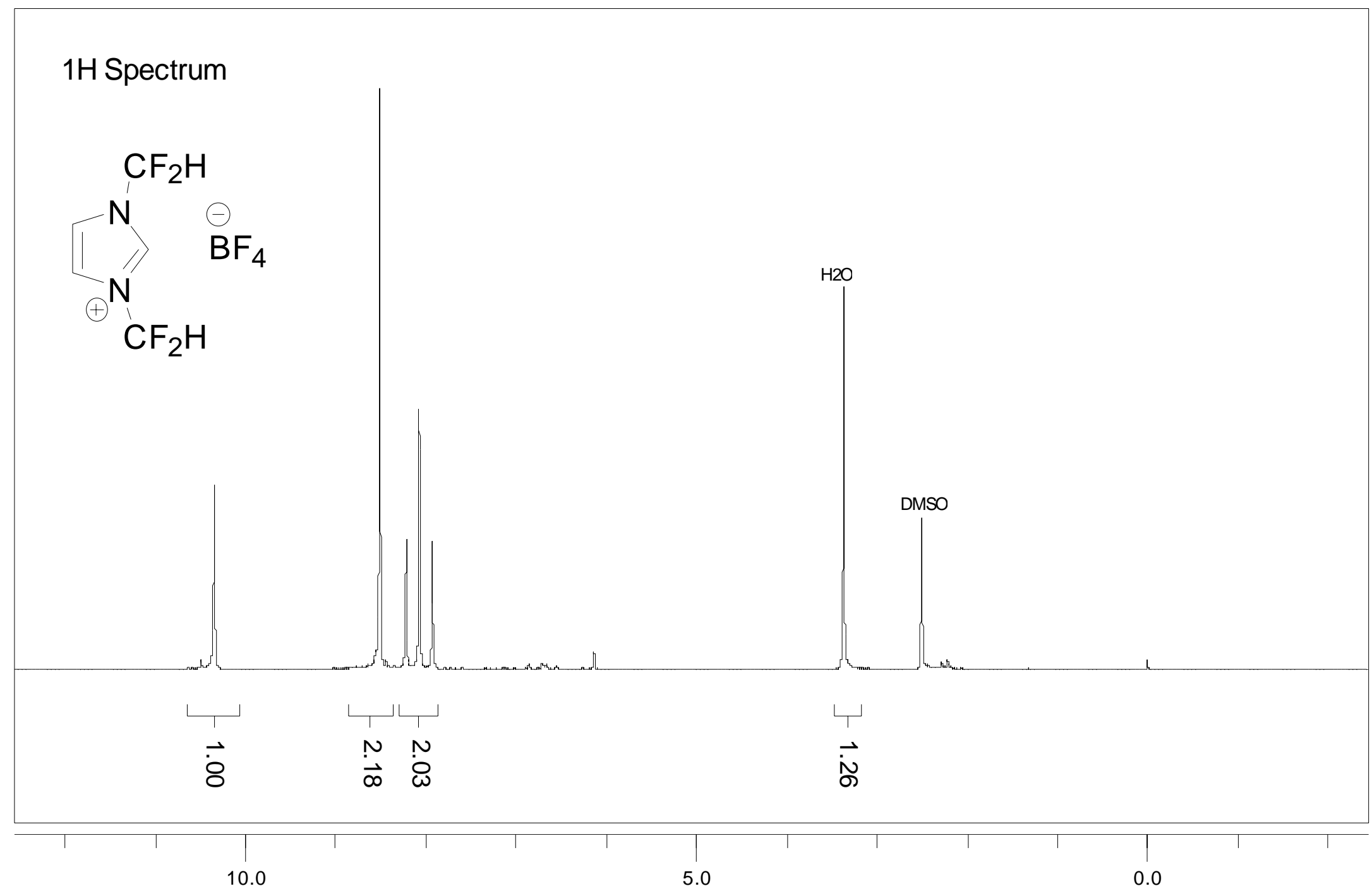

ppm (f1) 
$19 \mathrm{~F}$ Spectrum

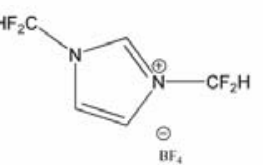

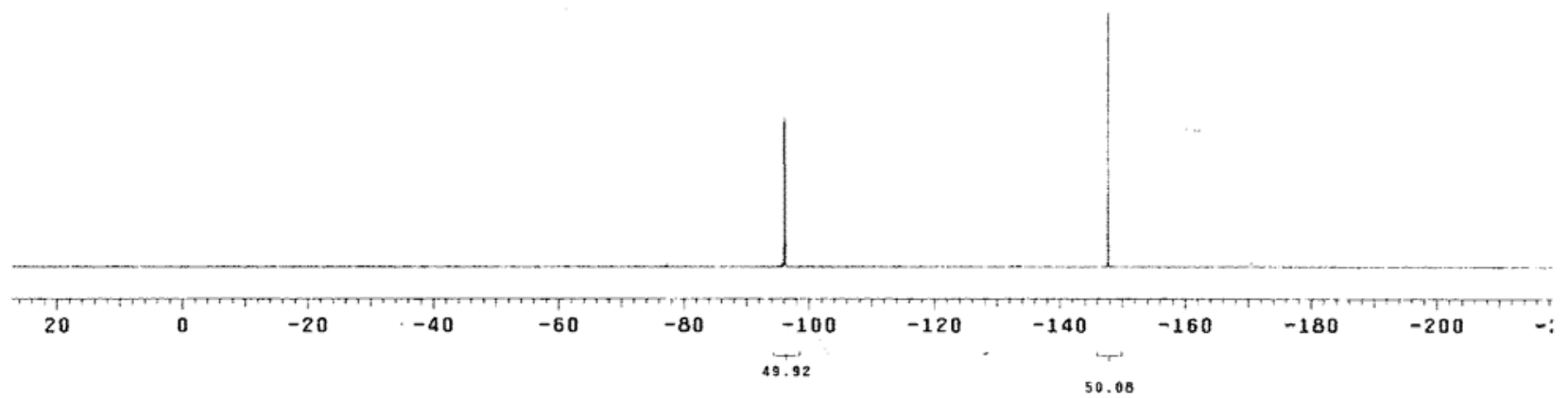

- S85 - 
19F Spectrum
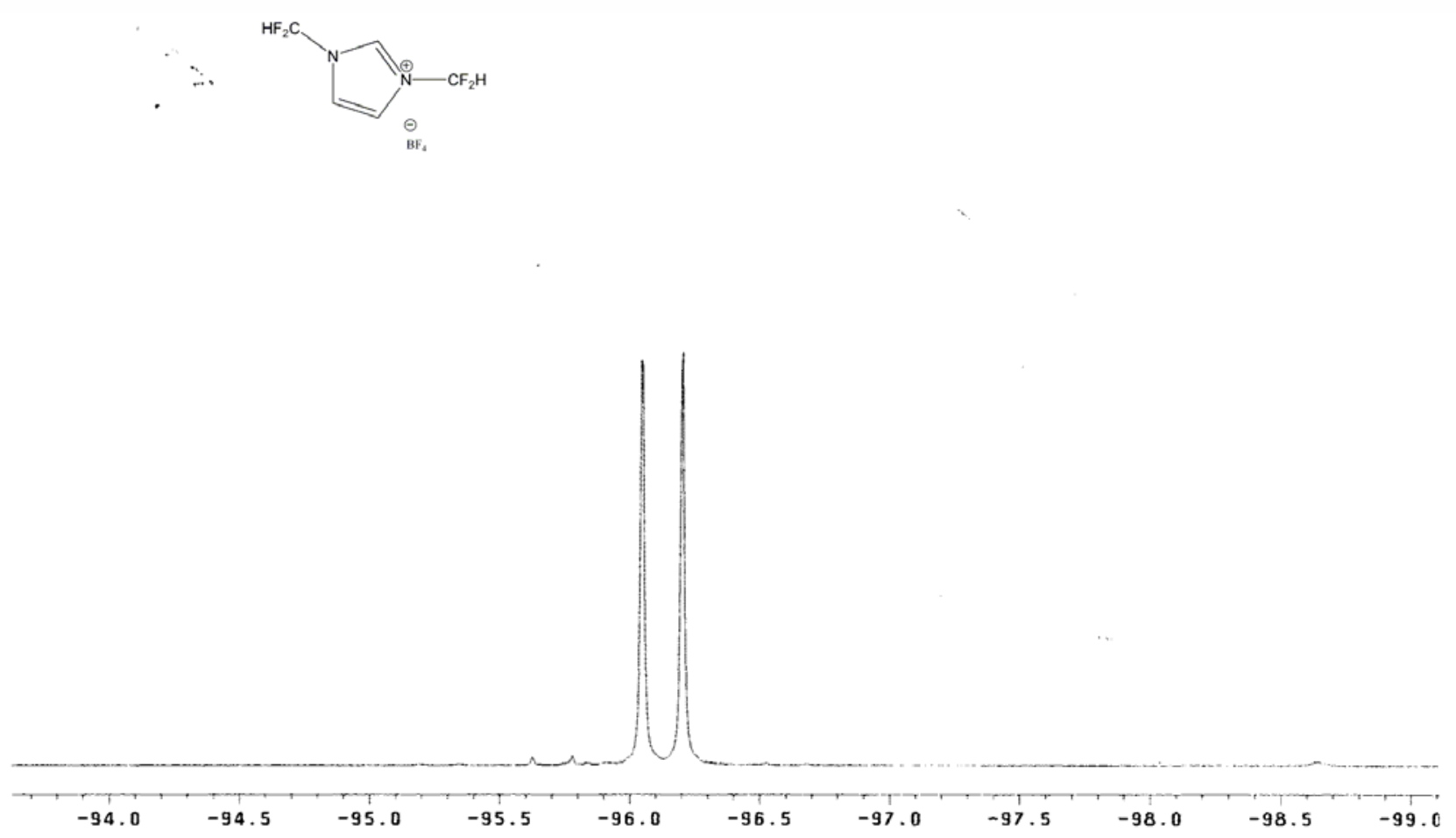

- S86 - 
19F Spectrum

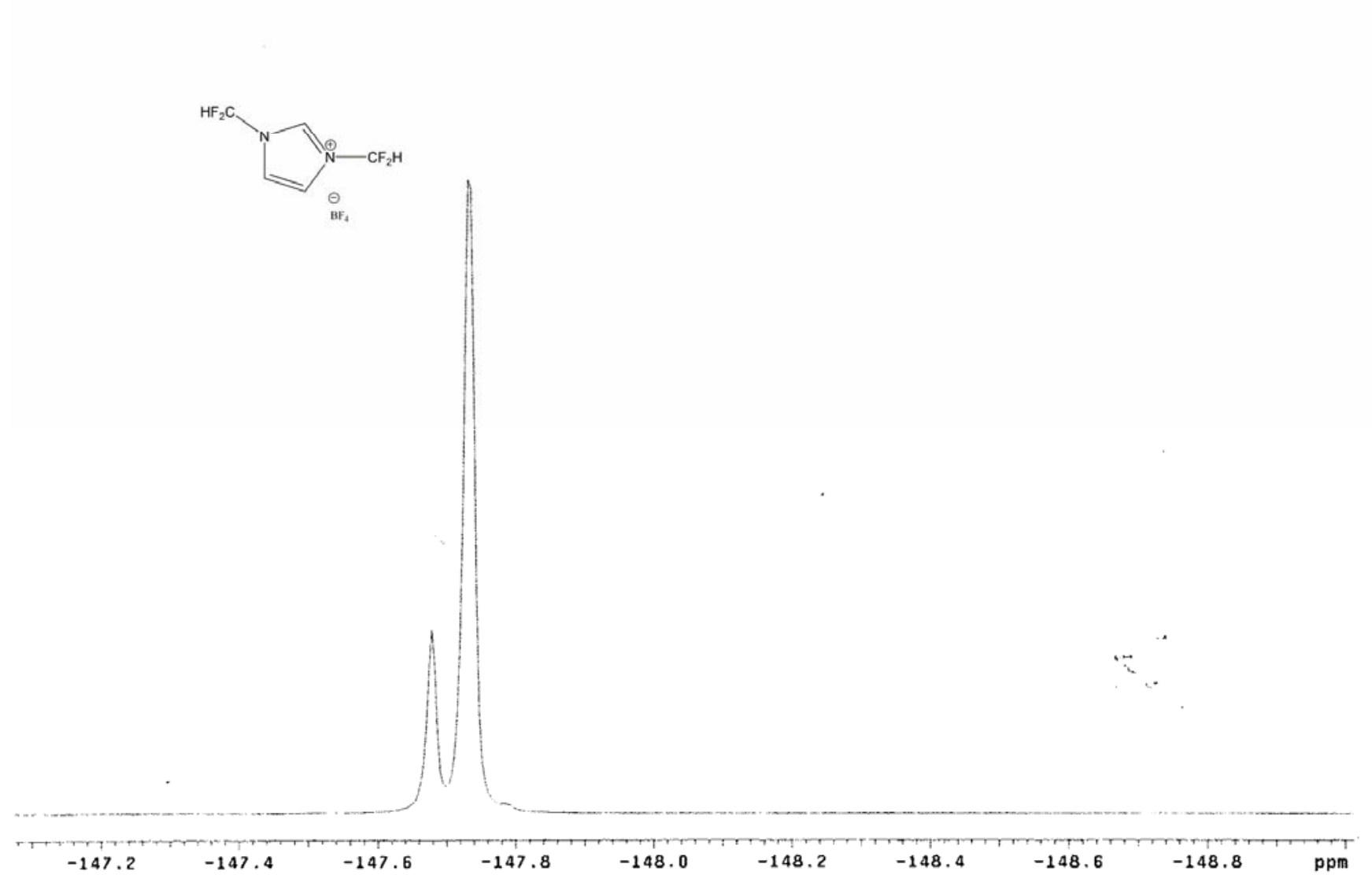




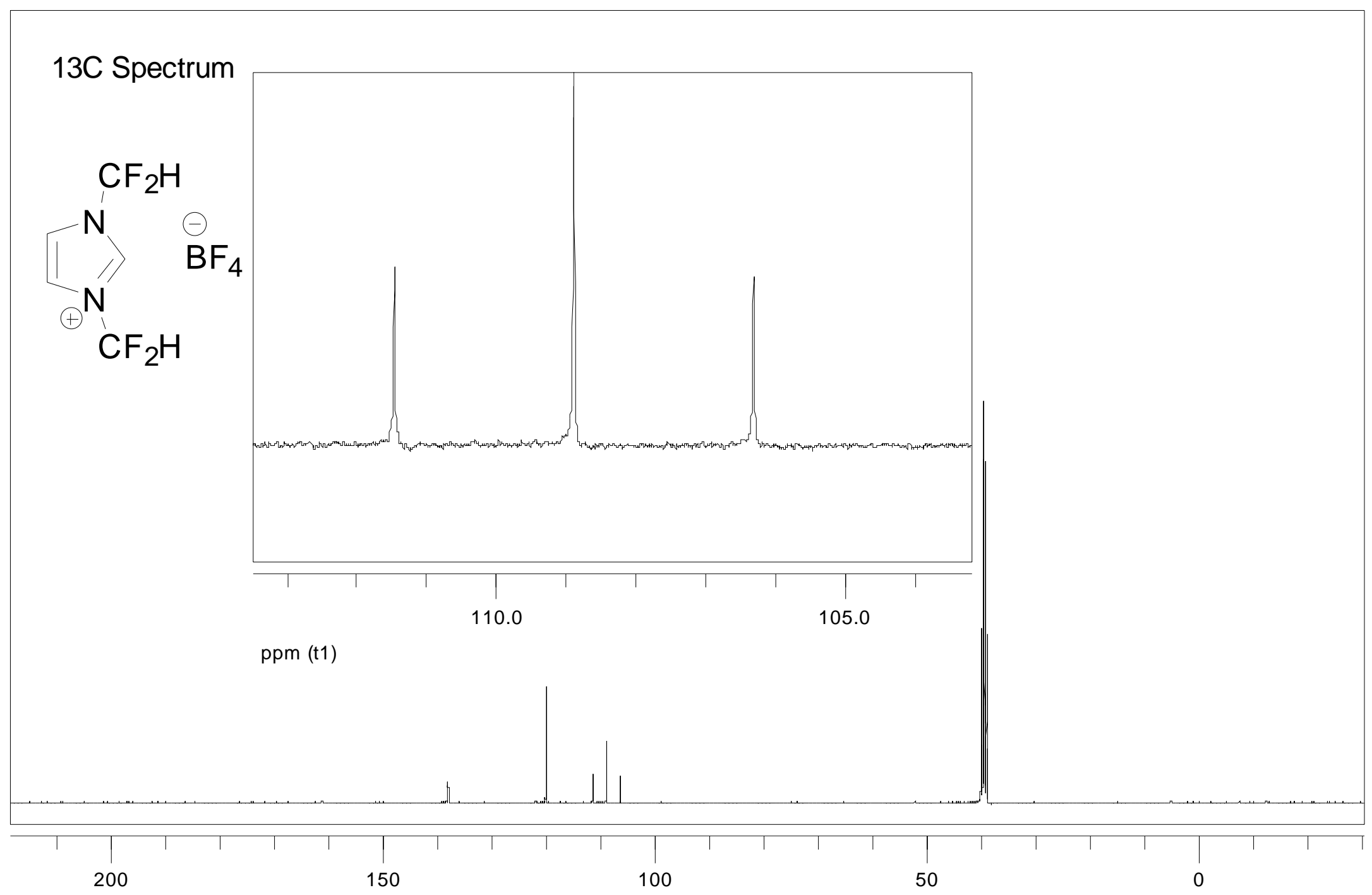

ppm (t1) 


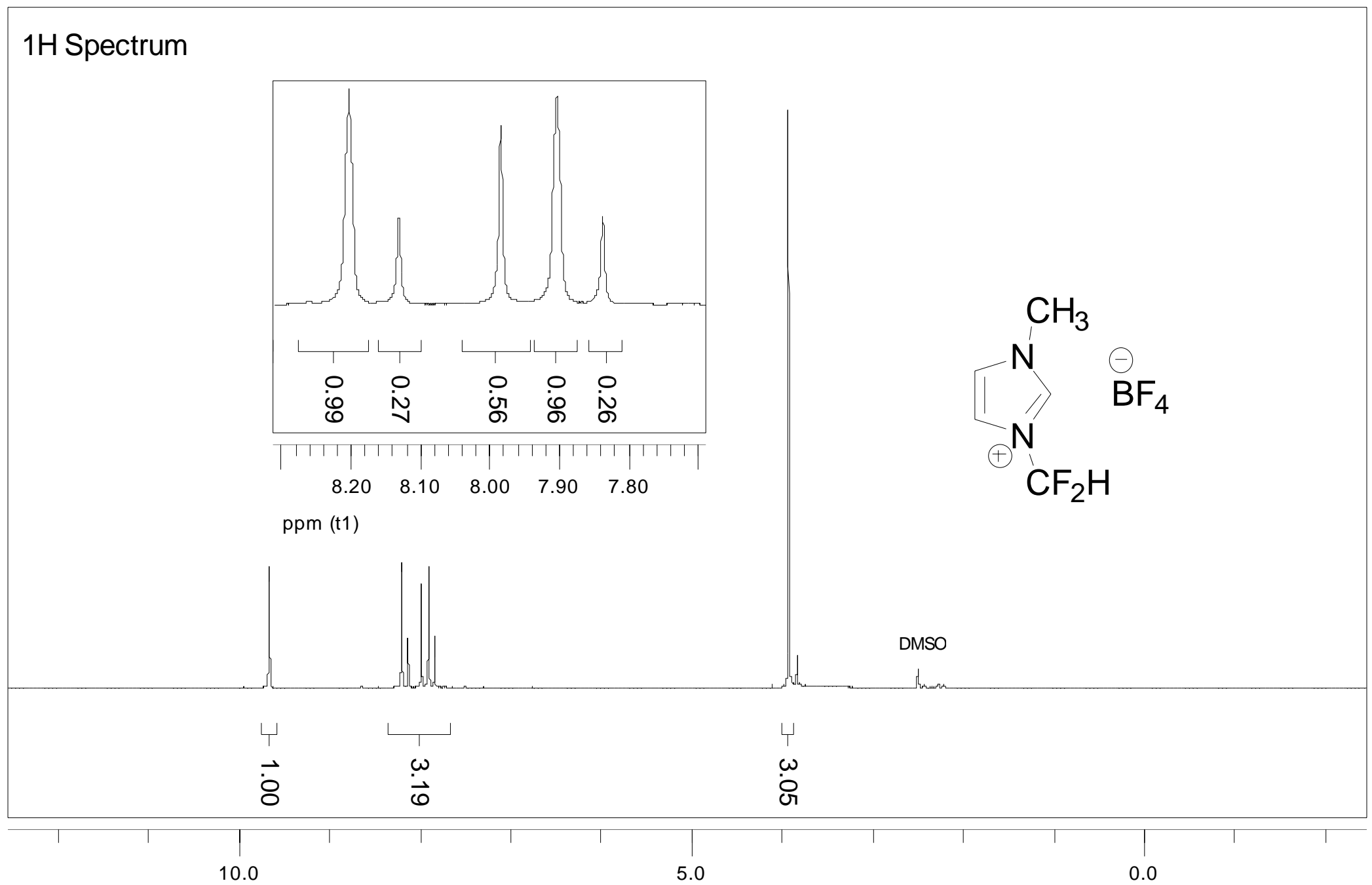

ppm (t1) 
19F Spectrum
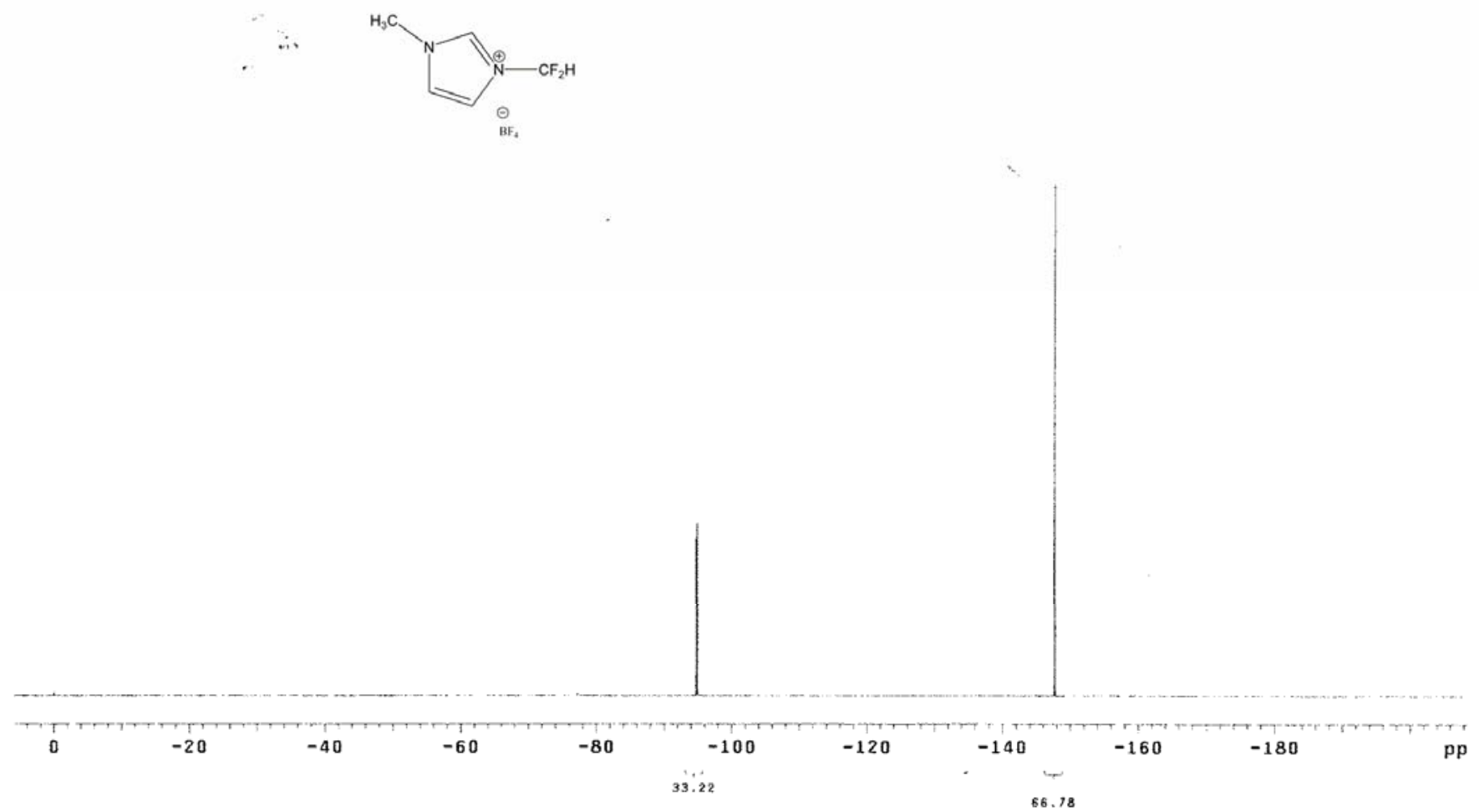

- S90 - 
$19 \mathrm{~F}$ Spectrum
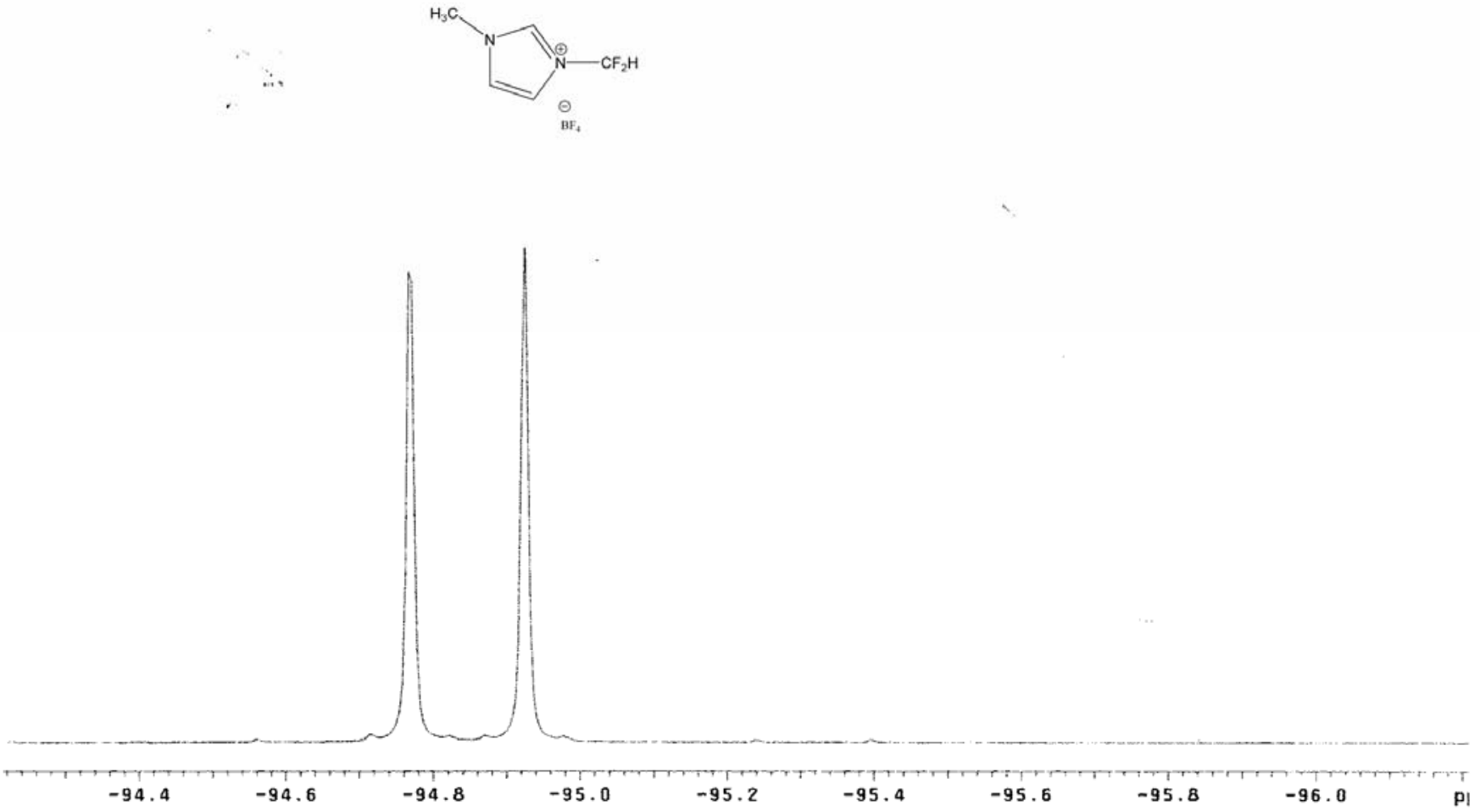

- S91 - 
19F Spectrum
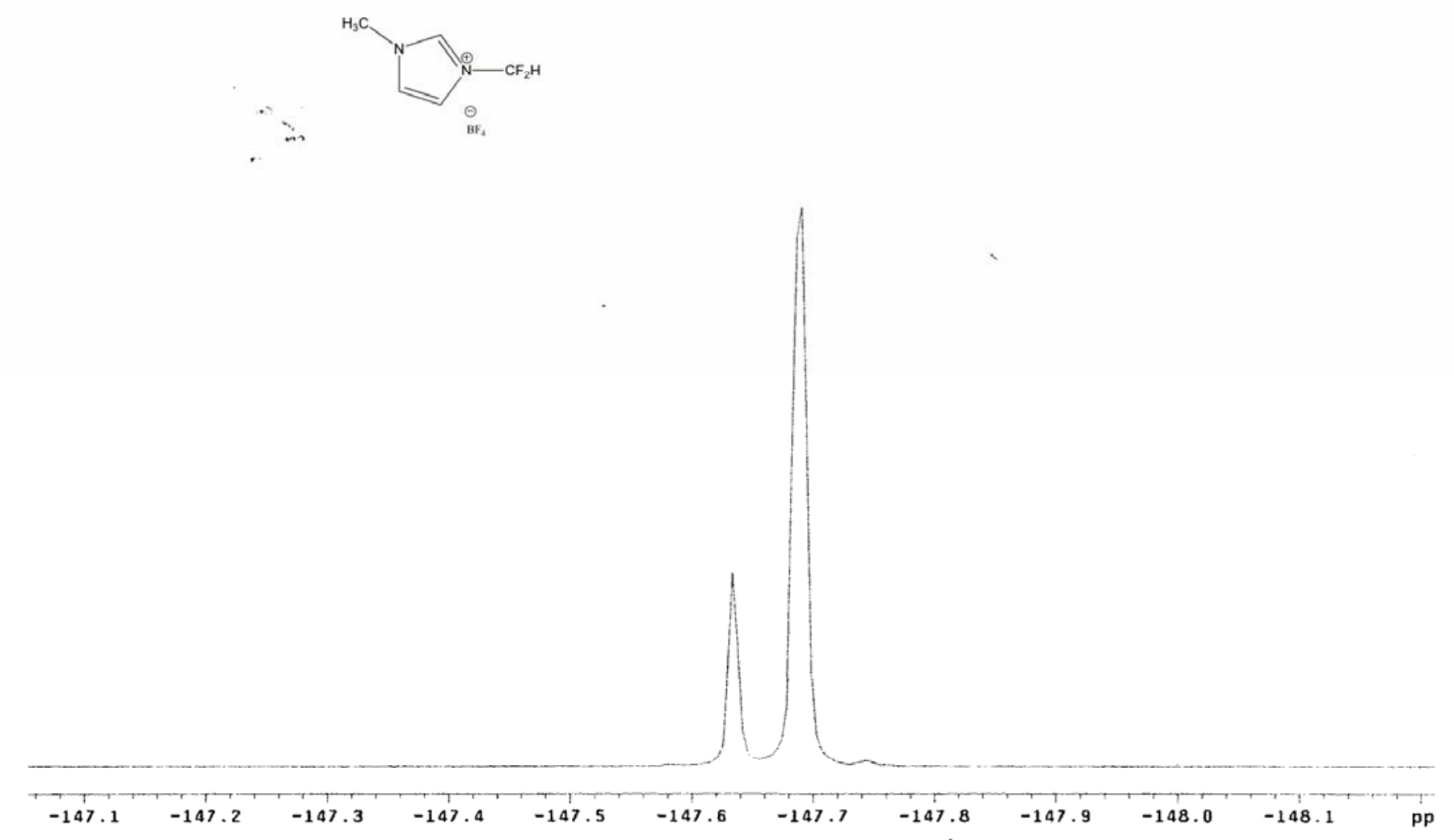
13C Spectrum

\section{$\mathrm{CH}_{3}$}

N

$$
\mathrm{BF}_{4}
$$

$\mathrm{N}$

$\mathrm{CF}_{2} \mathrm{H}$

200

150

ppm (f1) 


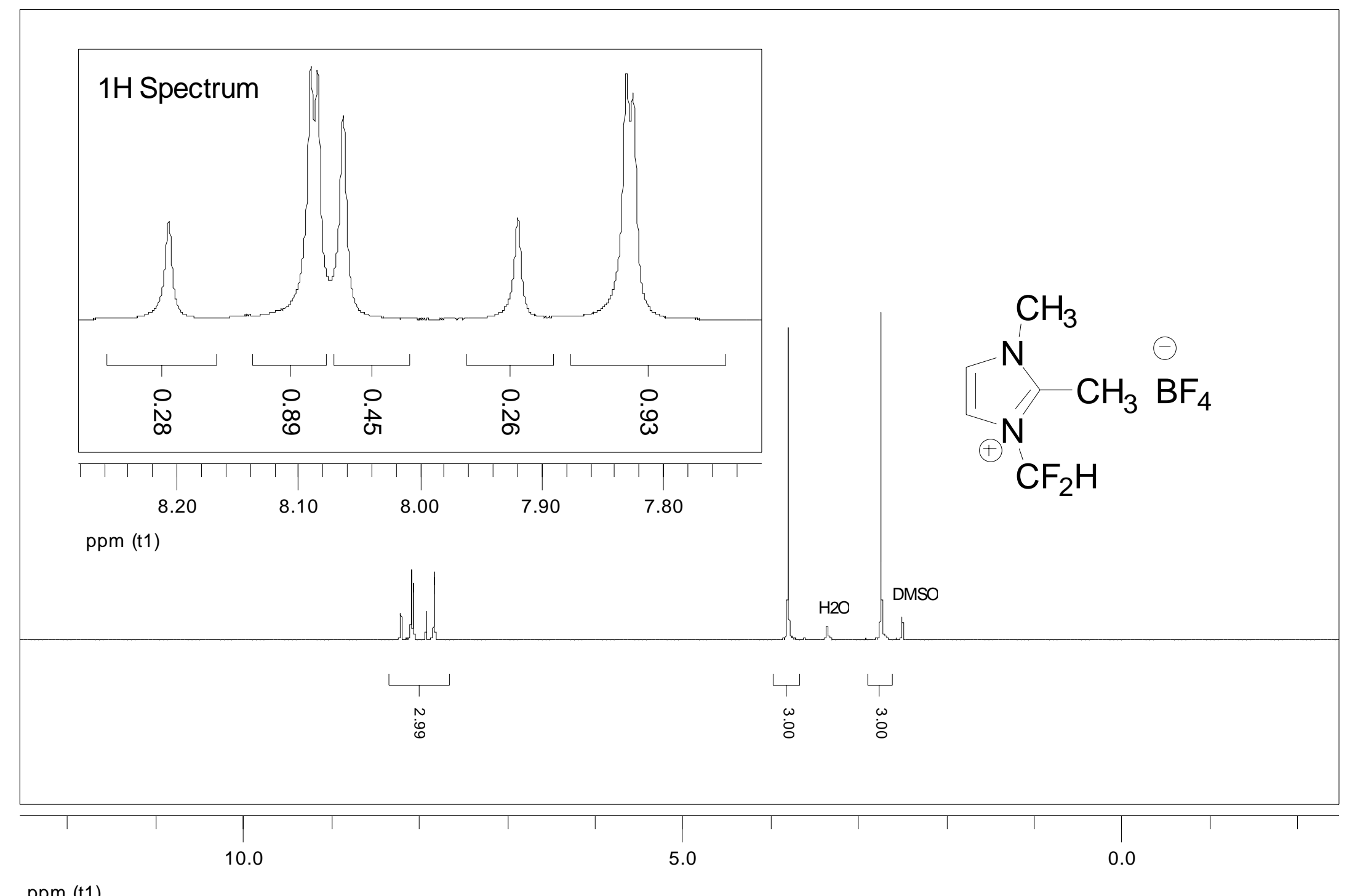

ppm (t1) 
19F Spectrum
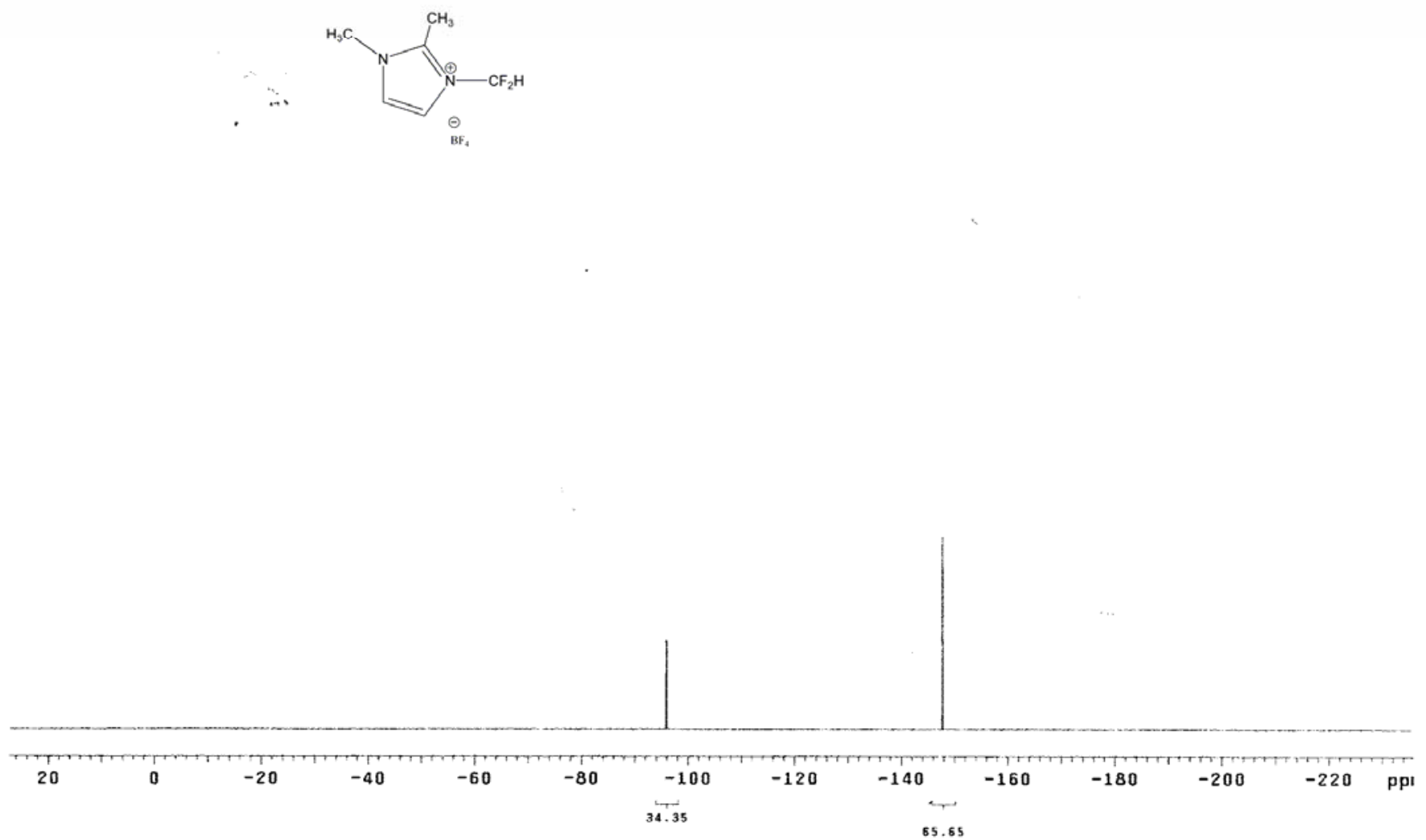

- S95 - 
19F Spectrum
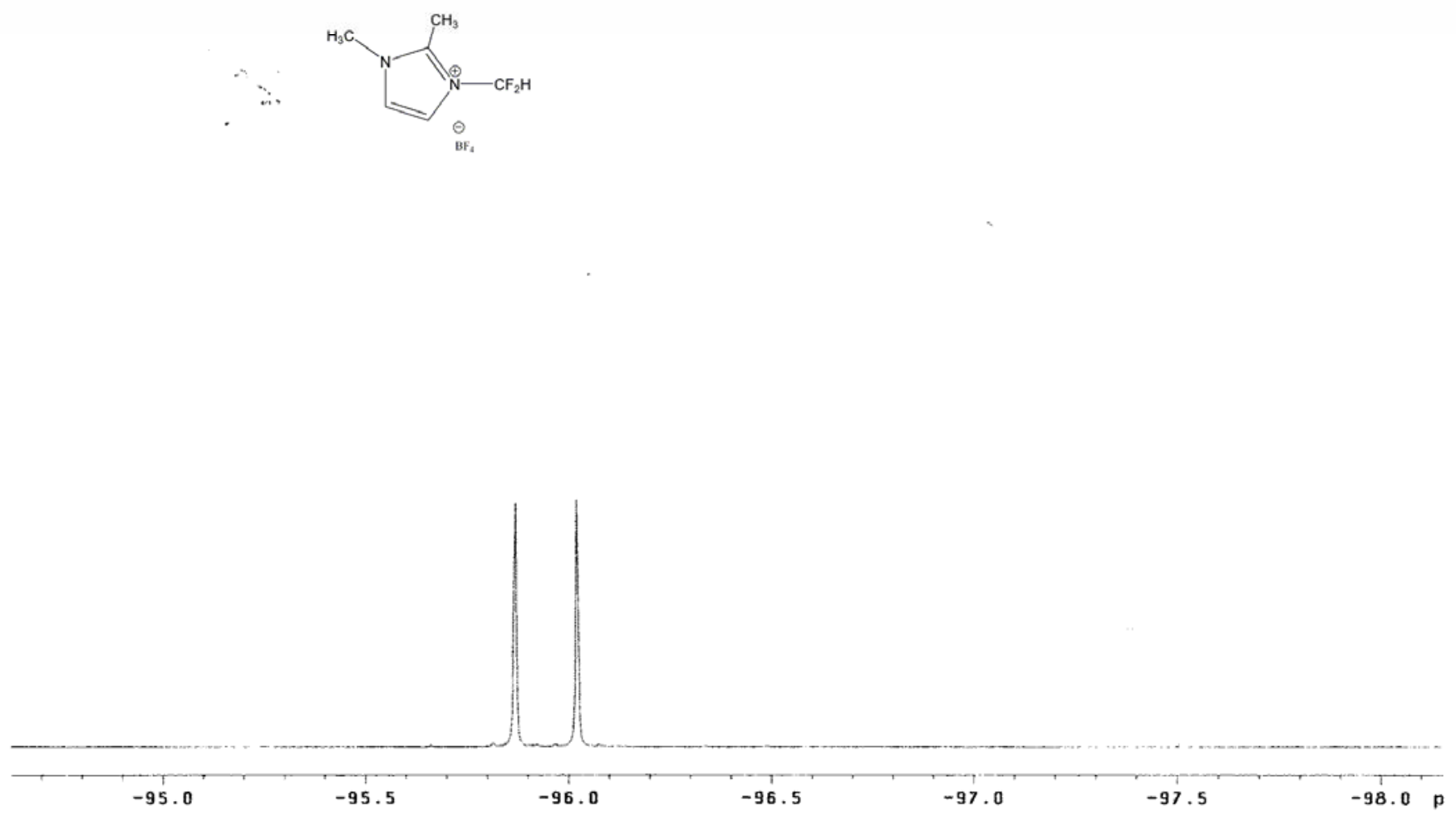
19F Spectrum
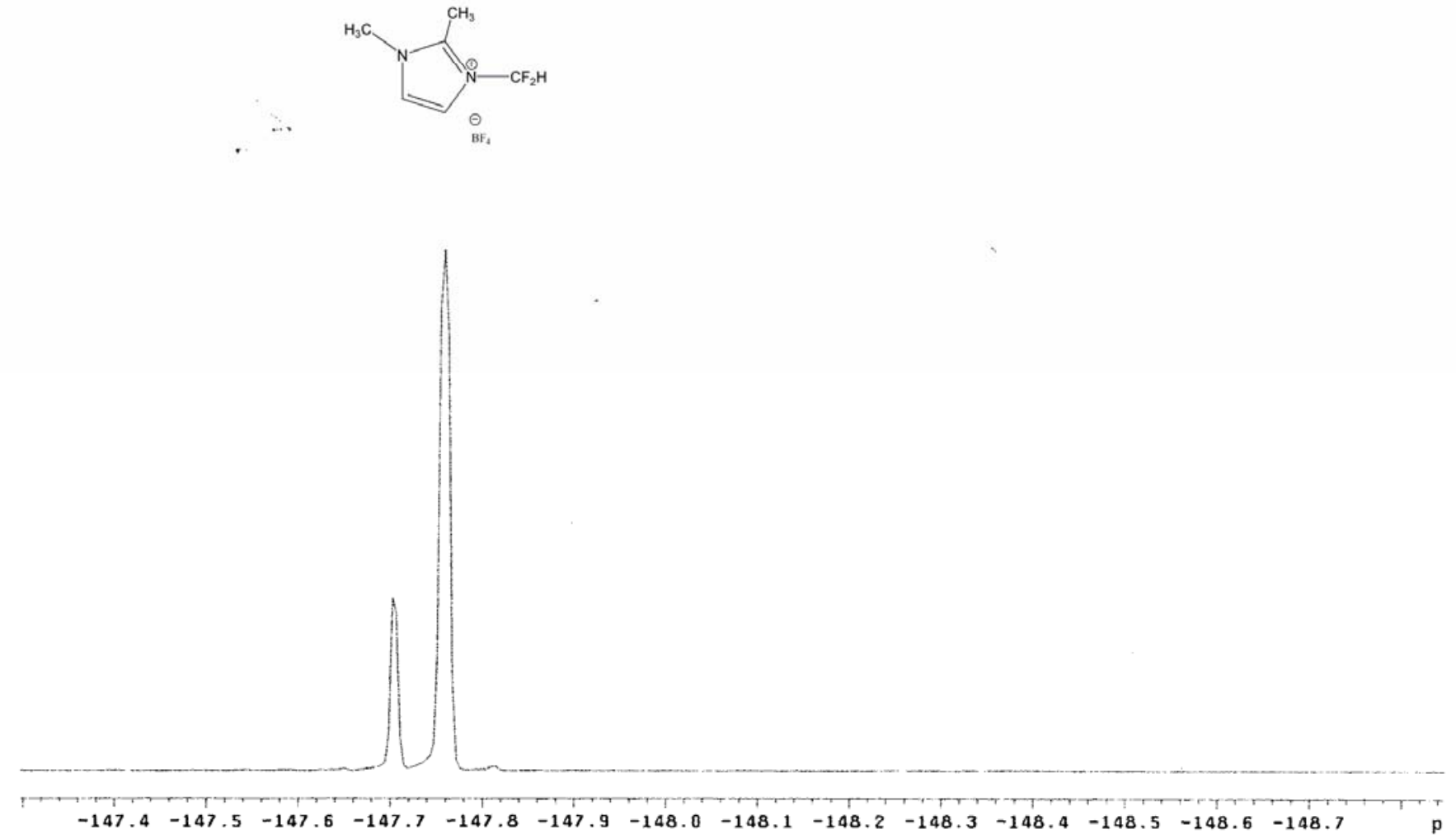

- S97 - 


\section{C Spectrum}

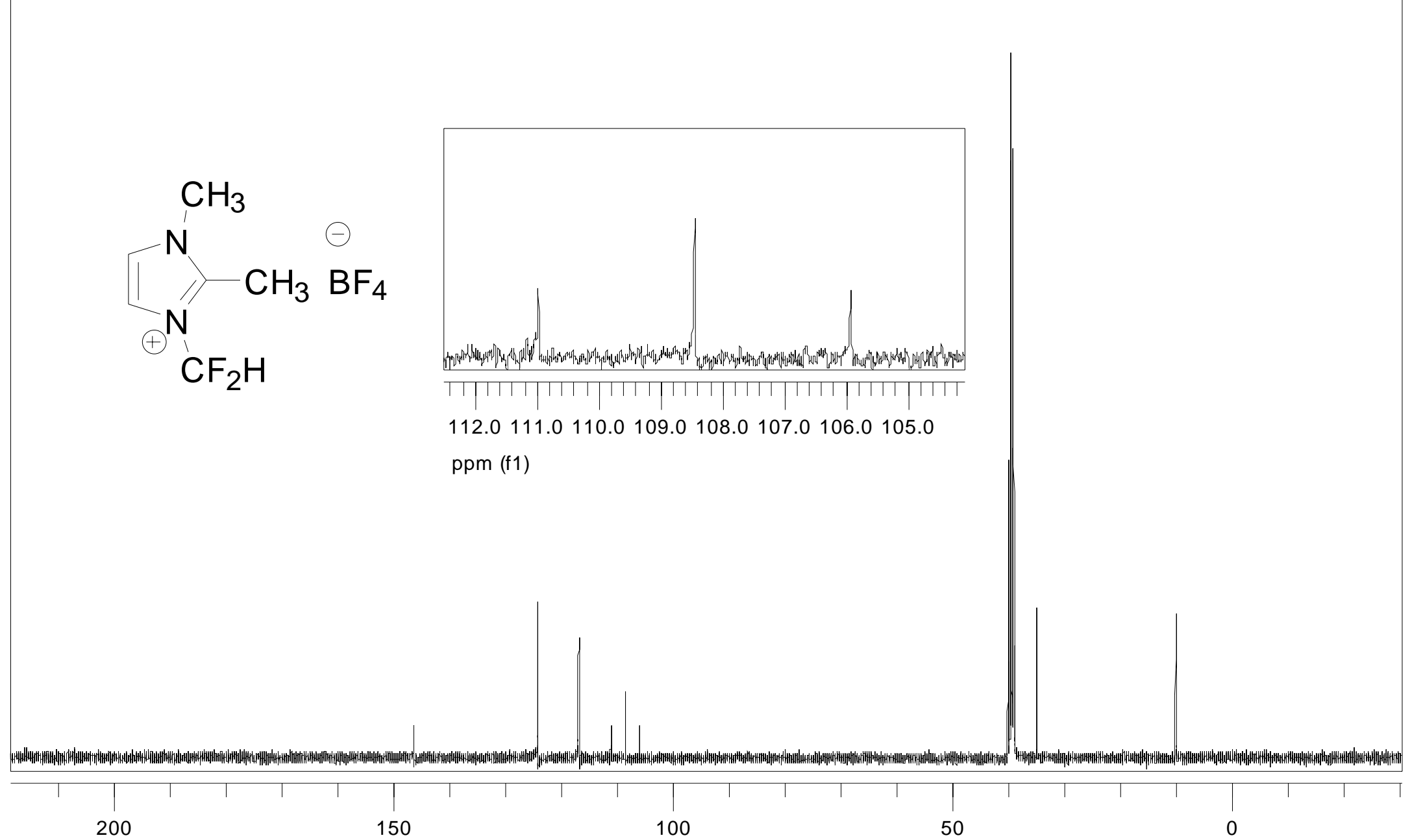

ppm (f1) 


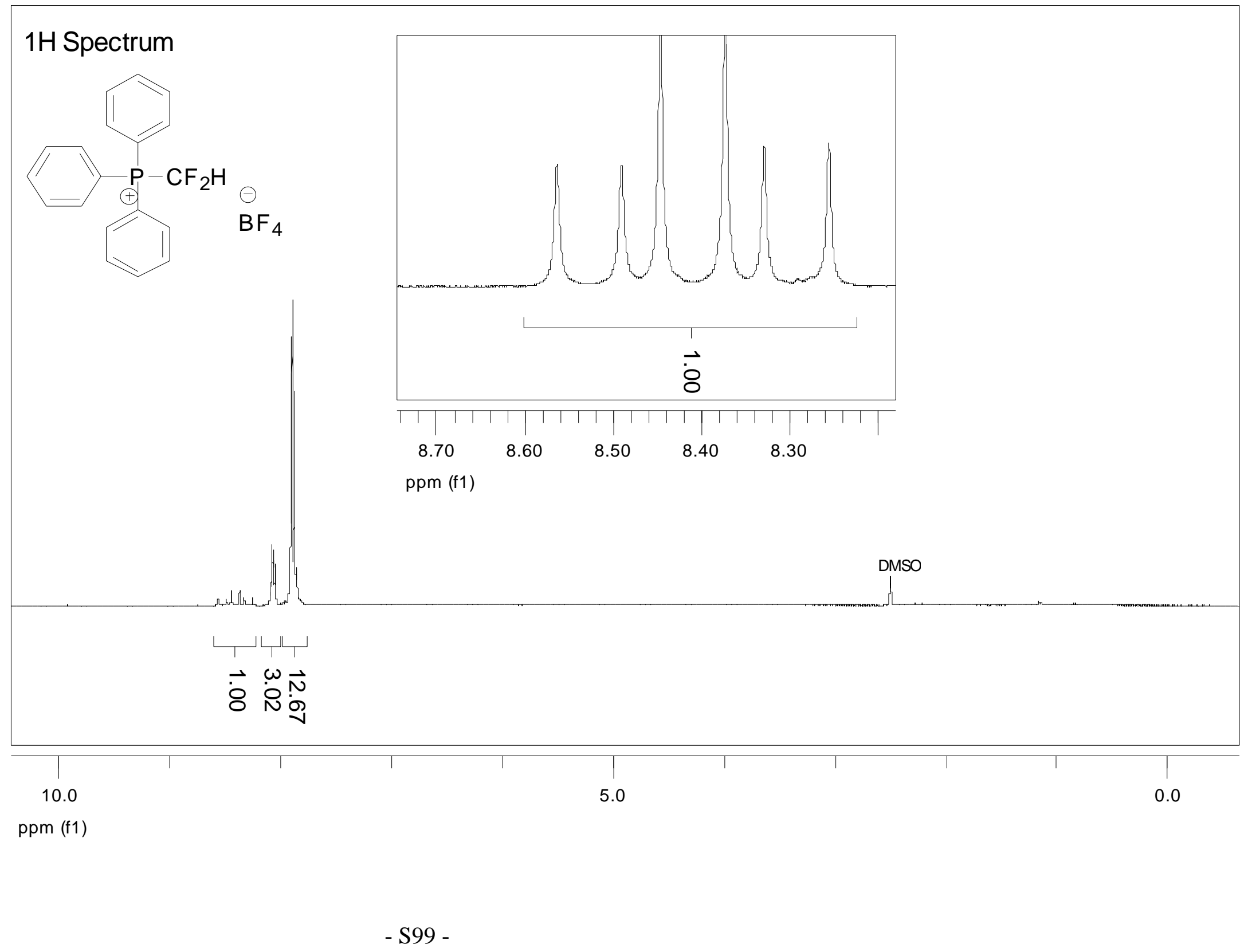


19F Spectrum
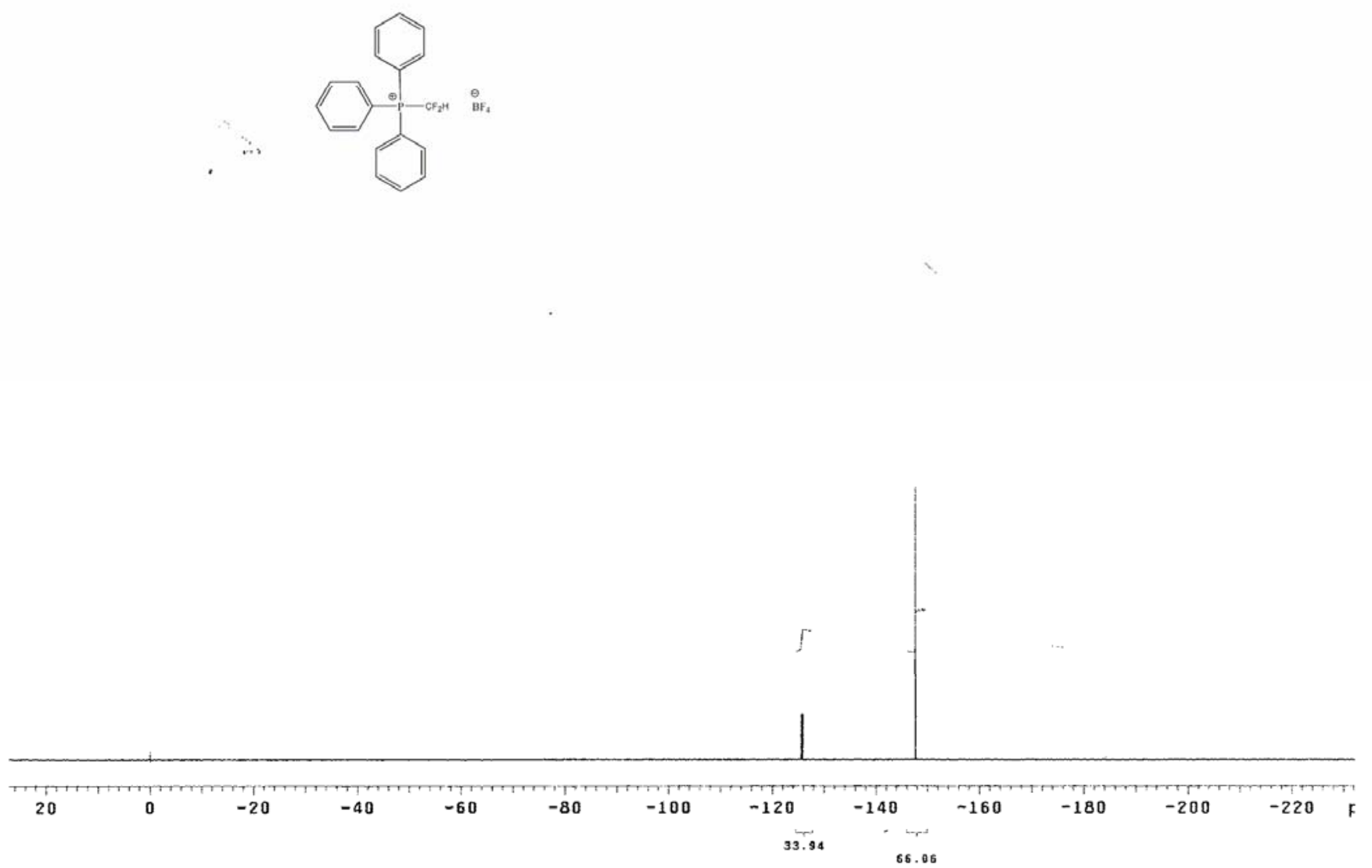

- S100 - 
19F Spectrum
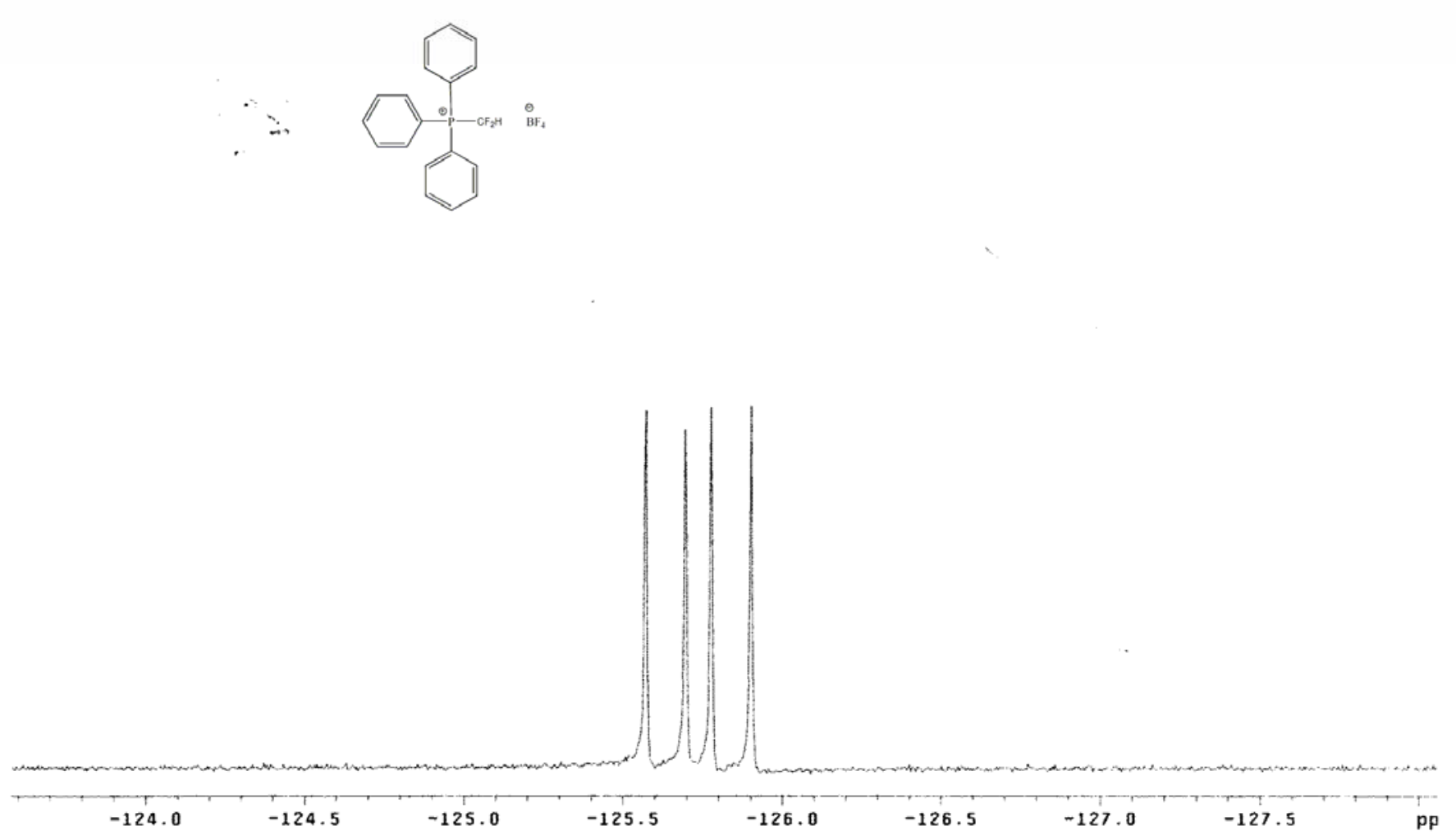

- S101 - 
19F Spectrum
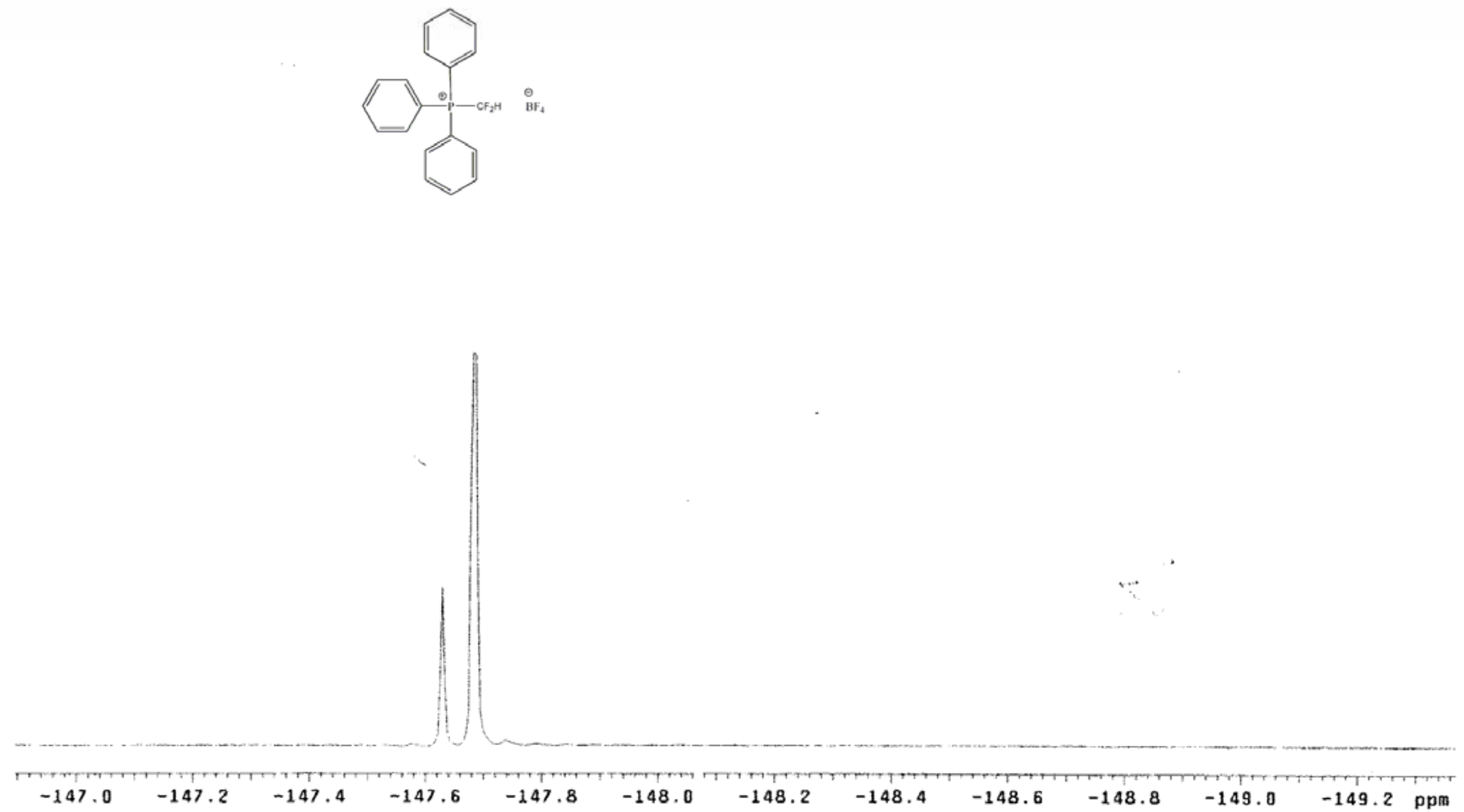

- S102 - 
31P Spectrum

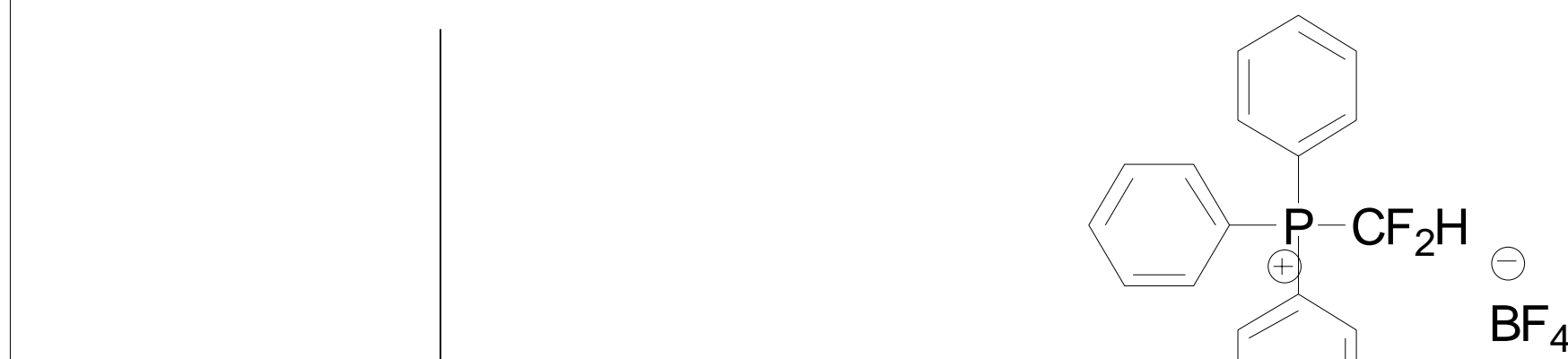

Fith

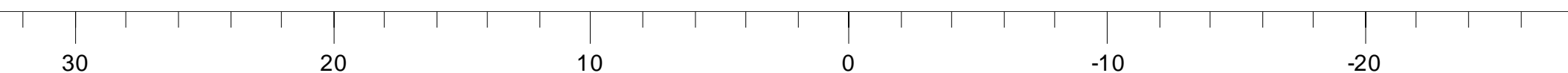
ppm (f1) 


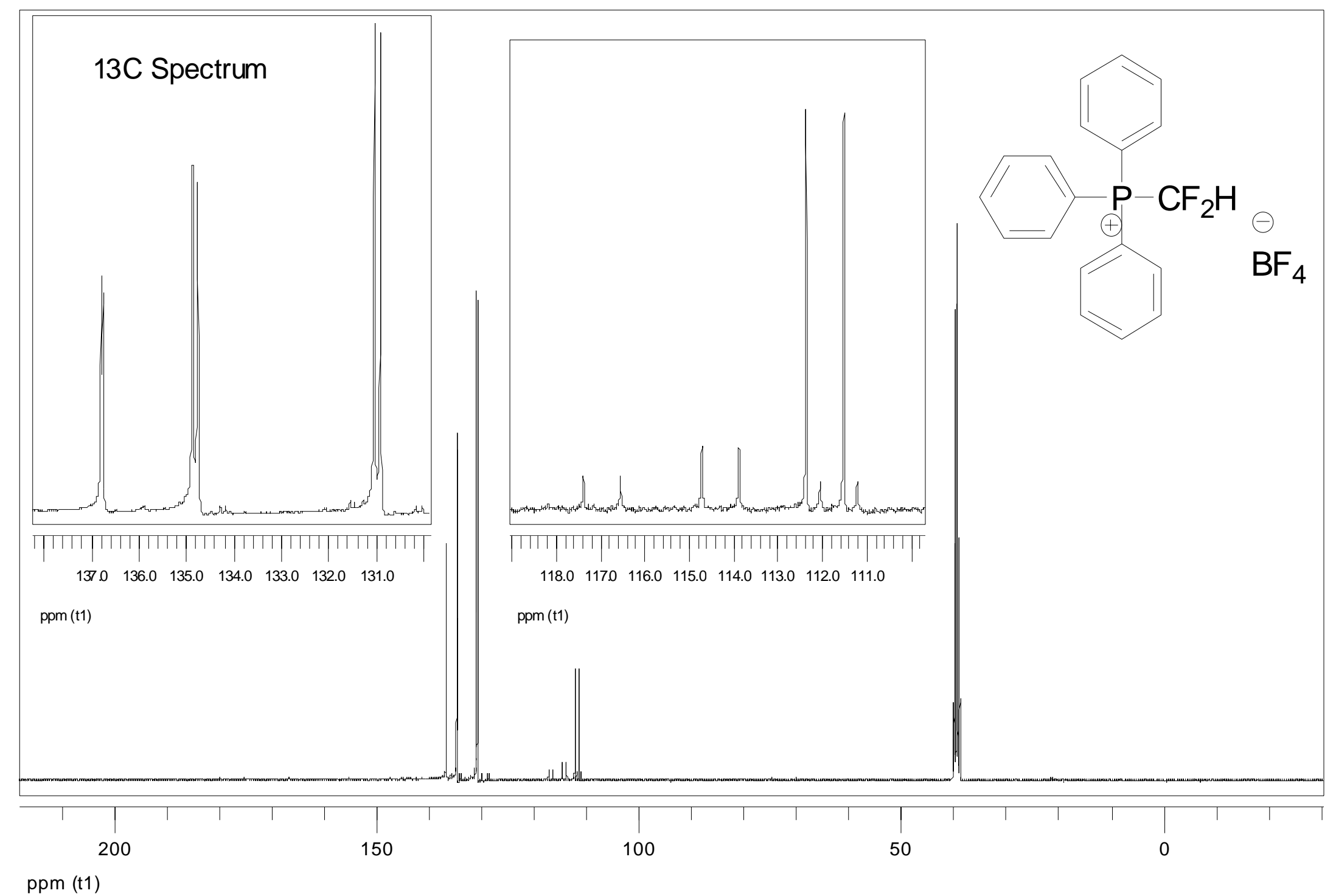

ppm (t1) 


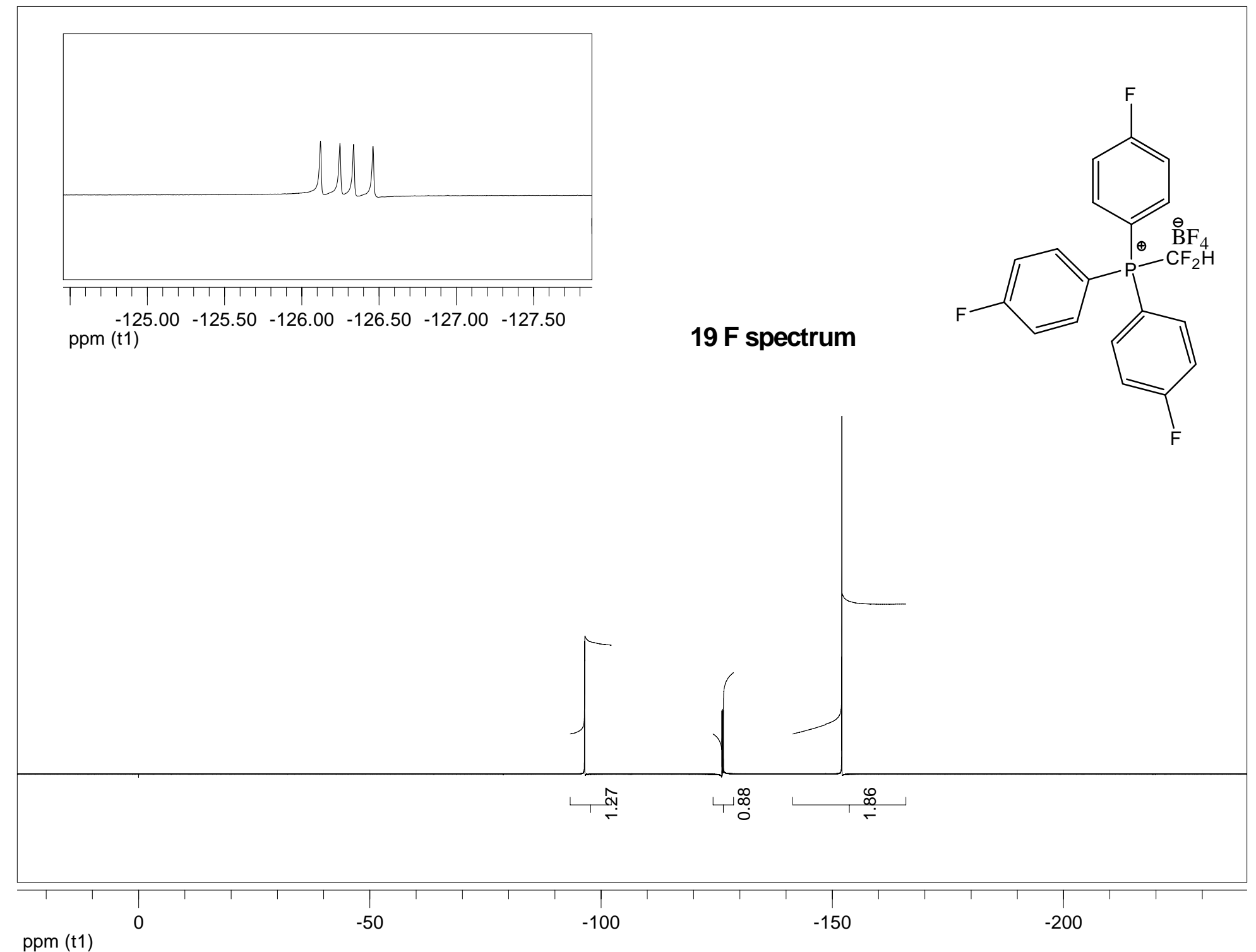

- S105 - 


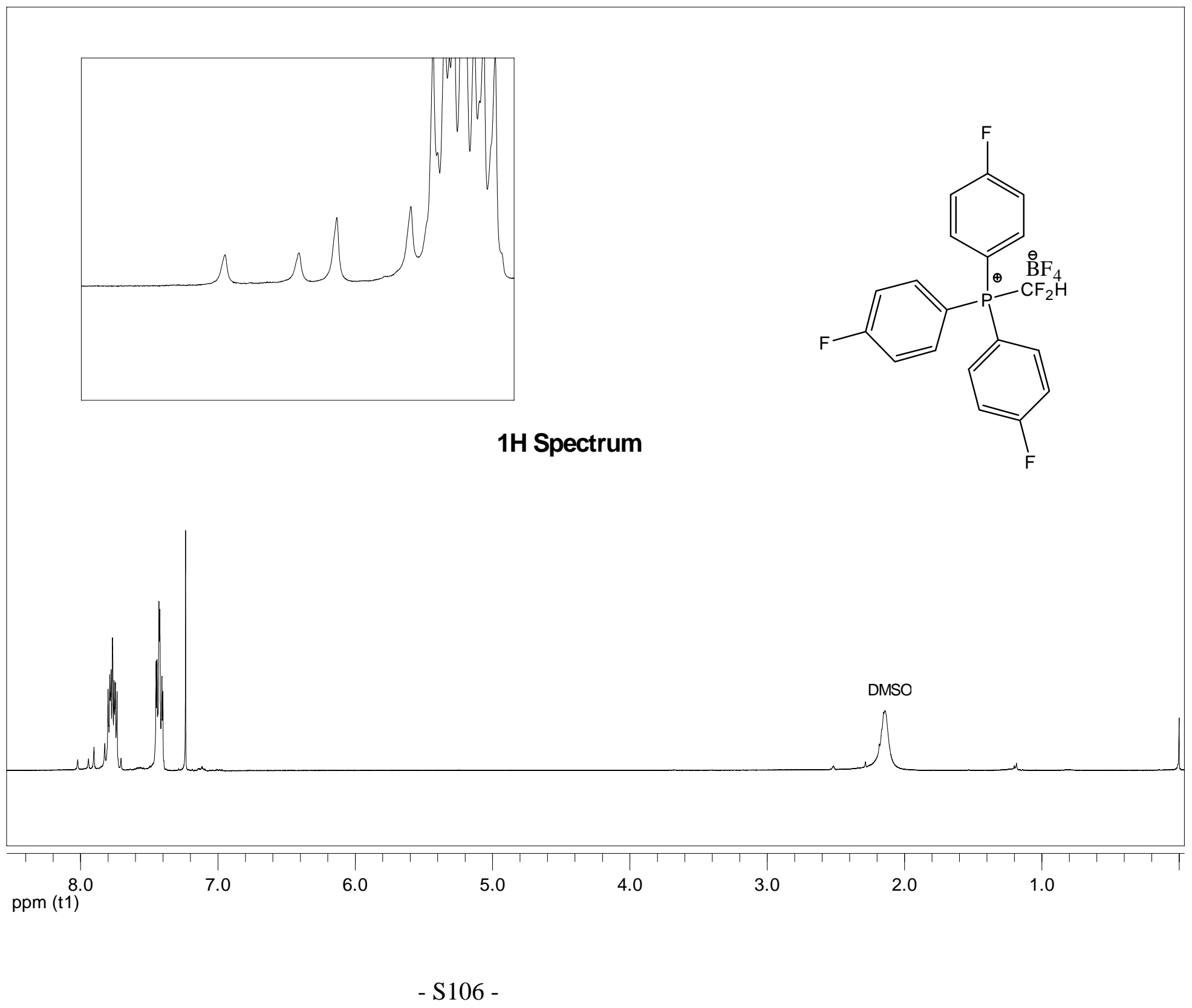




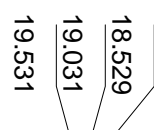

31 P spectrum

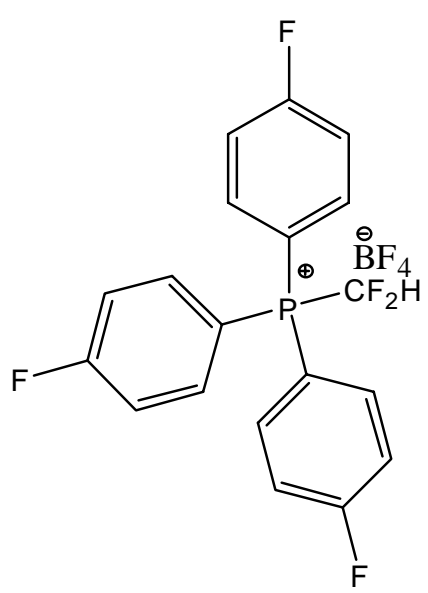

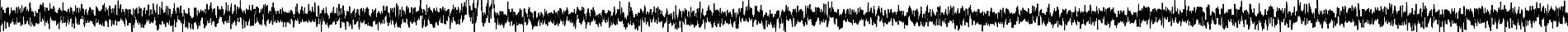

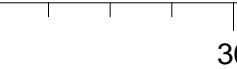

30

20

10

$-20$

ppm (t1)

$$
\text { - S107 - }
$$




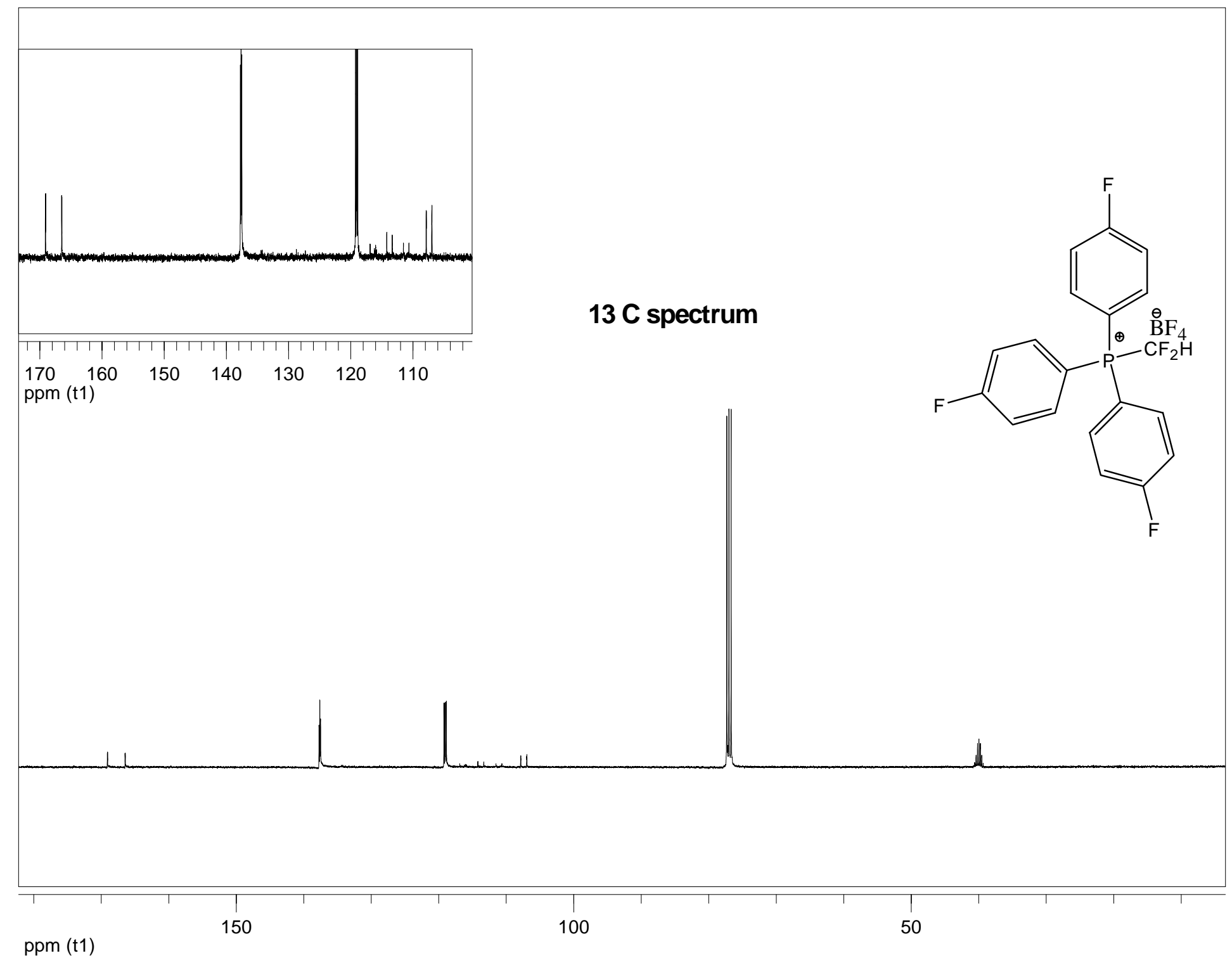

- S108 - 


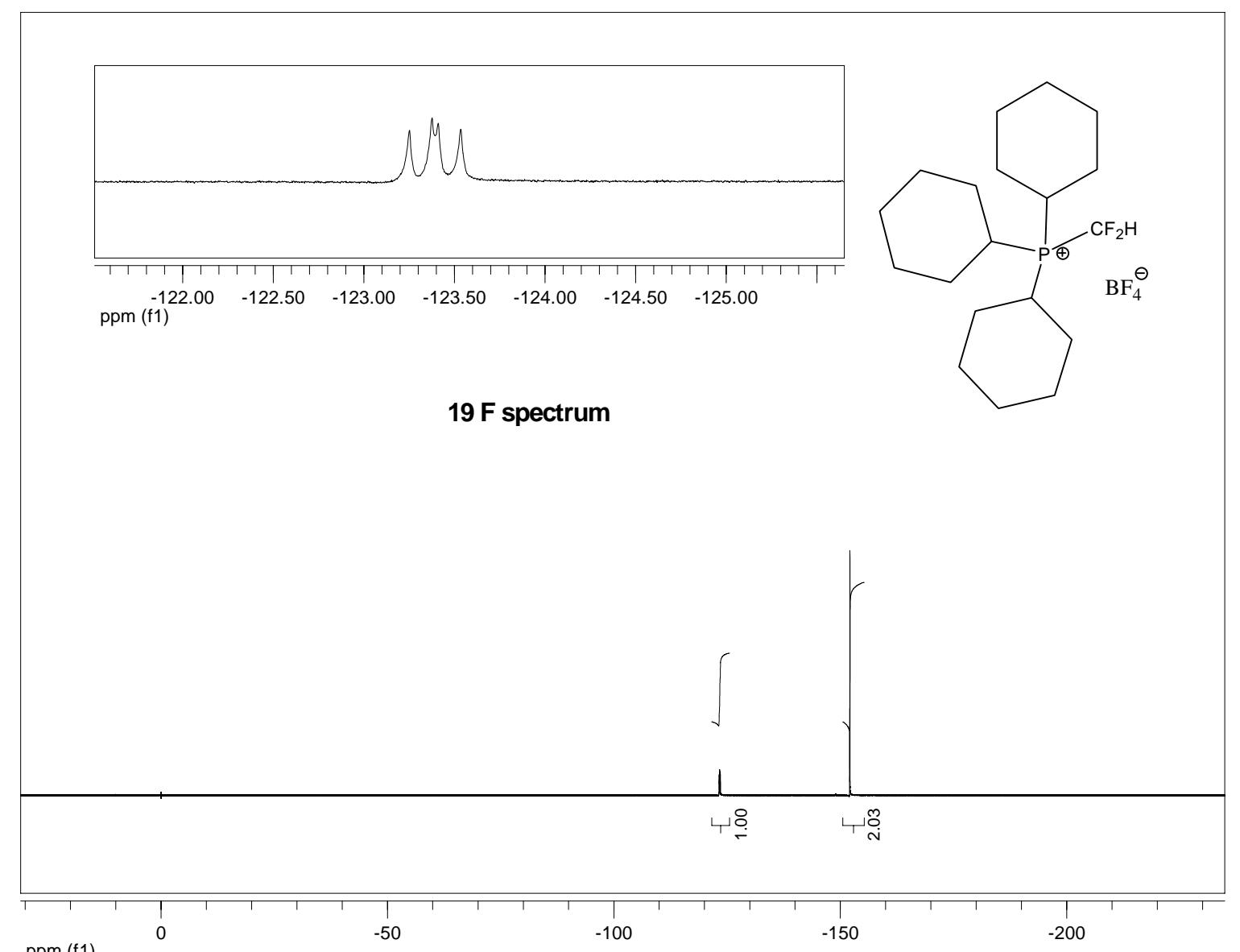

- S109 - 


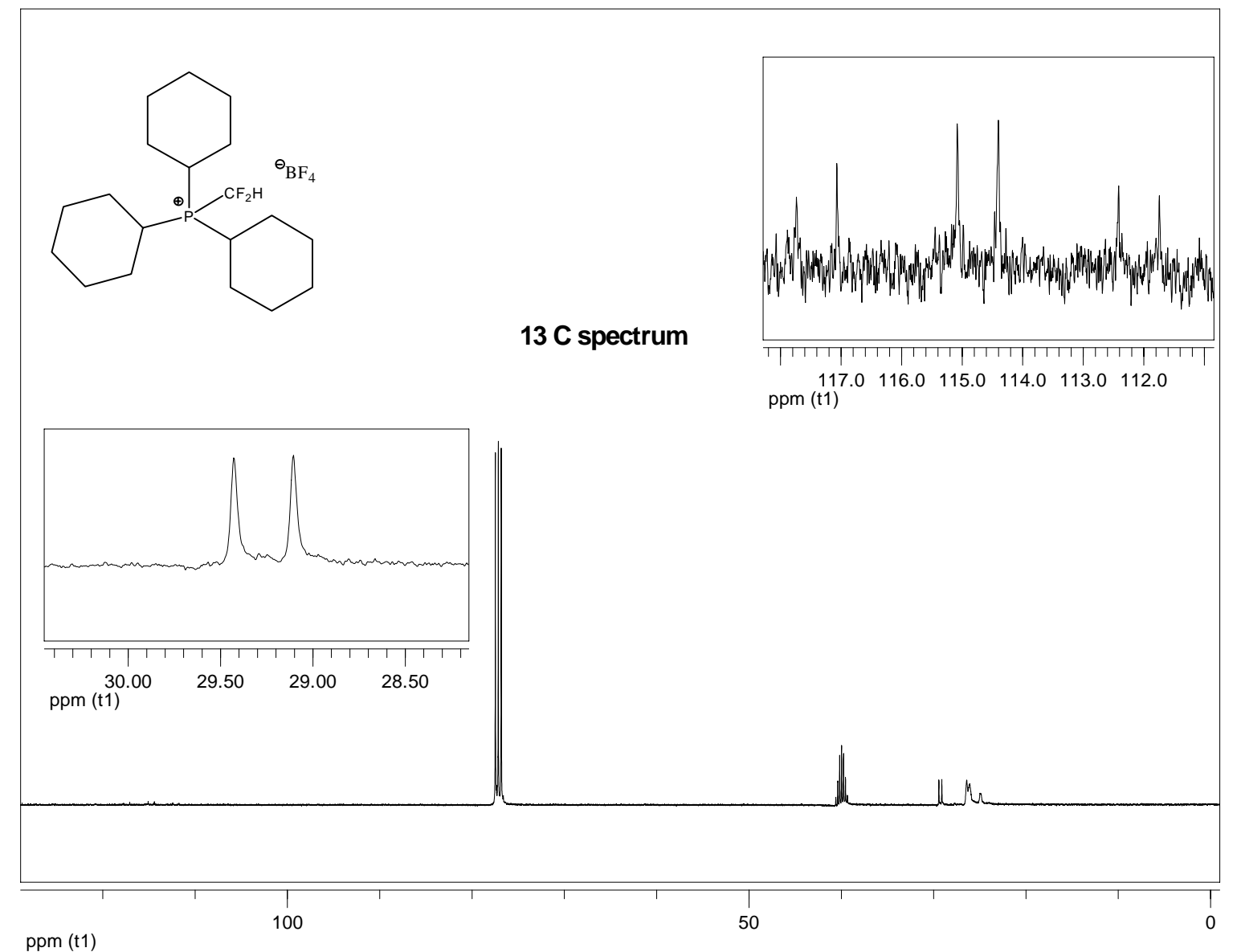

- S110 - 


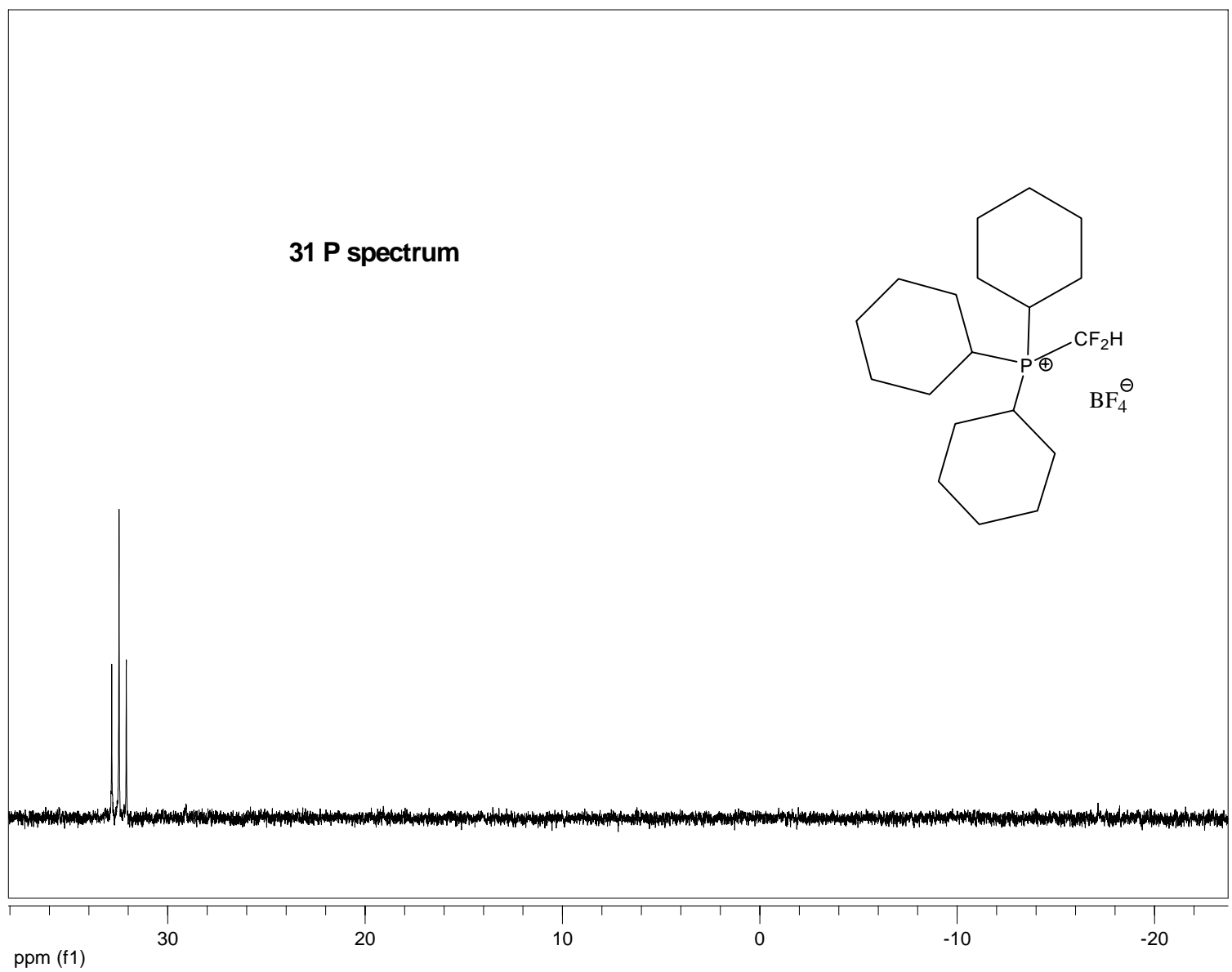

- S111 - 


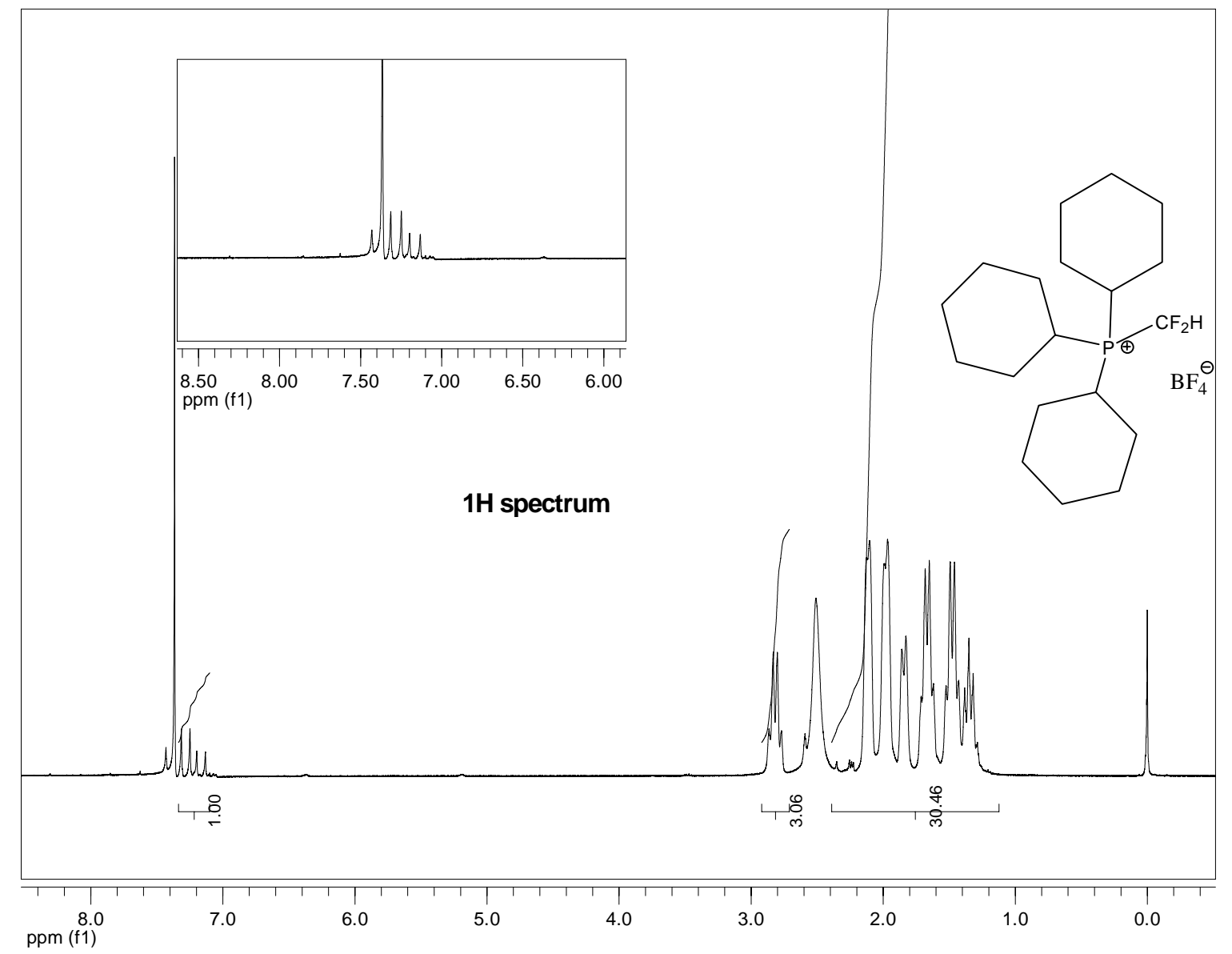

- S112 - 